\title{
DYNAMIC NUCLEAR-POLARIZATION STUDIES OF PARAMAGNETIC SPECIES IN SOLUTION
}

LBL- -14625

DE83 001533

Wayne Edward Glad

Materials and Molecular Research Division, Lawrence Berkeley Laboratory and Department of Chemistry, University of California

Berkeley, CA 94720

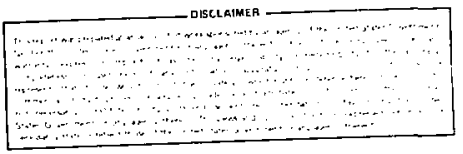

This work was supported by the Director, Office of Energy Research, Office of Basic Energy Sciences, Chemical Sciences Division of the U.S. Department of Energy under Contract No. DE-AC03-76SF00098. 
DYNAMIC NUCLEAR POLARIZATION STUDIES OF PARAY:AGNETIC SPECIES IN SOLUTION

\section{Contents}

Abstract ..................... v

I. Introduction .................. I

11. Electron Spin Relaxation in Liquids .......... 4

A. Mechanisms for $s=1 / 2 \ldots \ldots 4$

B. Mechanisms for $s \geq 1 \ldots \ldots$

III. Theory of Dynamic Nuclear Polarization . . . . . . . 15

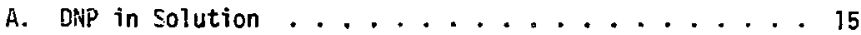

B. DNP in the Presence of Cnemical Exchange ....... 26

c. Measuring Spin Lattice Relaxation Times ...... . 30

IV. Flow Spectrometer ............... 36

A. Introduction ............... 36

B. Spectrometer . . . . . . . . . . . . 39

V. Flow Spectrometer Results ............ 48

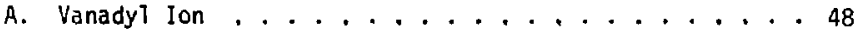

B. Copper Ethylenediamine ............ 64

c. Manganous Ion ................ 78

VI. Moving Sample Spectrometer . . . . . . . . . . 89

A. Introduction ................... 89

B. Sample Arrangement . . . . . . . . . . 90

C. Microwave Power ............... 93

D. NMk Spectrometer .............. 94

E. Experimental Timing ............... 96

F. Estimates of $\left\langle\mathrm{H}_{\mathrm{T}}^{2}\right\rangle_{\mathrm{s}} \ldots \ldots . . \ldots 9$ 
VII. Moving Sample Tube Results . . . . . . . . . . 104 A. Hexacyano-Chromium (III) Ion .......... 104

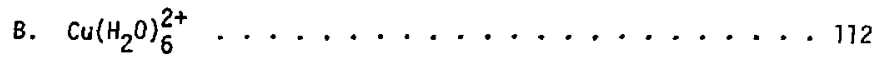
References................... 135 Acknowledgments ....................... 140 


\section{DYNAMIC NUCLEAR POLARIZATION STUDIES OF PARAMAGNETIC SPECIES IN SOLUTION}

Wayne Edward Glad

Materials and Molecular Research Division, Lawrence Berkeley Laboratory and Department of Chemistry, University of California

Berkeley, CA 94720

\section{ABSTRACT}

Dynamic Nuclear Polarjzation (DNP) was used to measure the electron spin lattice relaxation times, $T_{1}$, of transition metal ions in aqueous solution. Saturation which is induced in the electron spin system is transferred to the solvent proton spins by dipole-dipole interactiors. The change in the polarization of the proton spins is much larger than it is in the electron spins. The change in proton polarization is easily measured by proton Nuclear Magnetic Fesonance (MR).

In one experimental arrangement the sample solution was cont inuously flowed through a microwave cavity to the MR cojl. The MMR was observed with a continuous wave MMR spectrometer. In a second arrangement the whole sample tube was moved from within the microwave Cavity to the NMR coil in less than 40 ms by a blast of compressed air. The MR was then observed with a pulse-Fourier-transform spectrometer. Witil the second arrangement a mean-square microwave magnetic field at the sample of more than $10 \mathrm{G}^{2}$ is obtainable with $14 \mathrm{~W}$ of microwave pcwer.

Measurements of DNP at $9 \mathrm{GHz}$ were made on aqueous solutions of $\mathrm{vo}^{2+}$, $\mathrm{Mn}^{2+}, \mathrm{Cr}(\mathrm{CN})_{6}^{3-}, \mathrm{Cu}^{2+}$ and $\mathrm{Cu}(\mathrm{e} \text { thylenediamine })_{2}\left(\mathrm{H}_{2} \mathrm{O}\right)_{2}^{2+}$ jons from 3-60"C. It was also possible to observe ONP on resolved protun resonances from mixed water-acetonitrile solutions of $\mathrm{vo}^{2+}$ and $\operatorname{Cr}(\mathrm{CN})_{6}^{3-}$ ions. 


\section{Introduction}

Magnetic resonance spectroscopy has proven to be a valuable tool in the solution of physical, chemical, and biological problems. New applications of magnetic resonance are constantly being found and new instruments are being built to increase the generality of its use. Magnetic resonance, however, is more than a tool; it has been the object of intensive study in its own right. The desire to understand the number, position, intensity, and shapes of magnetic resonance signals has provided ample opportunity for the development of theories describing the interactions of paramagnetic species with their physical and chemical environments. In this work, one magnetic resonance phenomenon, Dynamic Nuclear Polarization (DNP), has been used as a tool to investigate another magnetic resonance phenomenon, electron sp in relaxation in solution.

DNP is a process whereby the polarization which is induced in an electronic spin system is transferred to a nuclear spin system. The electron polarization is induced by the application of a strong microwave field at the resonance frequency of the Electron Paramagnetic Resonance (EPR) transition.

In this work, DNP is used to aid in the measurement of electron spin relaxation rates which are difficult or impossible to measure by other means. Other uses of DNP can include Nuclear Magnetic Resonance (MR) sensitivity enhancement and studies of molecular motic $n$ in solut ions.

Bloch (1946) characterized the relaxation of a spin system with two relaxation times. The first, which he called $T_{1}$, the spin-lattice 
relaxation time, is the time constant for the return to equilibrium of the $z$ component of the magnetization after it has been perturbed away from thermal equilibrium. It describes the time dependence of the population of the sp in states. The second relaxation time, $T_{2}$, the spin-spin relaxation time, is the time constant for the disappearance of magnetization which has been induced in the $x-y$ plane. It describes the disappearance of phase coherence in the ensemble of spins. In EPR spectroscopy, $T_{2}$ for well-resolved lines is easily determined from the widths of the 1 ines. The determination of $T_{1}$ is usually much more difficult.

Two things are required for paramagnetic relaxation in solution. The first is a perturbation which can operate on the spin wavefunctions of the system. The second requirement is a motion which generates small oscillating magnetic fields to make this perturbation time-dependent and allows the flipping or dephasing of the spins. By studying the magnitude and temperature dependence of the relaxation rates, it is possible to learn something ahout the nature of both the perturbation and the motion which are responsible for the relaxation.

The pioneering work on relaxation in solution was that of Bloembergen, Purcell, and Pound (1948). They found that the motion responsible for the time dependence of the dipole-dipole interaction which was responsible for the relaxation of protons in water could be treated by using classical theories of diffusion which involved correlation functions for the random motion which decayed exponentially with respect to time. Measurement of the relaxation times could thus 
yield correlation times which are indicative of the speed and type of motion responsible for relaxation.

We are interested in the electron spin relaxation times of transition metal ions in solution. Understanding of the mechanisms of relaxation of these iors can yield infomation about the ir electronic structure in solution and their inferaction with the solvent. The magnitudes of the relayation times are also useful to other workers who are interested in the effect of paramagnetic ions on the ralaxation times of nuclei in the solution. Many measurements of $T_{2}$ have been made on transition met. 1 ions in solution, but of ten $T_{1}$ is the relaxation time of interest to those investigating nuclear relaxation. In addition, knowledge of both $T_{1}$ and $T_{2}$ is useful in determining the mechanisms responsible for relaxing the electron spin, since many interactions do not contribute equally to $T_{1}$ and $T_{2}$.

The present work was undertaken in order to measure $T_{1}$ values for some transition metal ions for which the relaxation mechanisms were not completely understood, with the hope of aiding in the understanding of the electron spin relaxation of these ions. Some ions for which the relaxation mechanisms are reasonably well understcod were also studied. This was done to test the applicability of using DNP to measure $T_{1}$, since this method is not in general use. 


\section{Electron Spin Relaxation in Liquids}

A large number of theories have been proposed to describe electron spin relaxation in liquids, but only a faw of them have been verified experimentally. Those theories which seem to have applicability to the ions studied in this work are discussed in this section. Only a simple description of the mechanism and the results of the theoretical calculations will be given here. The original papers should be consulted for details.

The electron spin relaxation of transition metal ions is dominated by the influence of the spin-orbit coupling. Spin orbit coupling produces magnetic anisotropies, which can cause relaxation when they are modulated by molecular motion. Also, perturbation of the electronic orbital wavefunctions caused by interactions with the surrounding molecules can be transferred to the electron spins through the spinorbit coupling to cause sp in relaxation. The dominant relaxation mechanisms for transition metal ions with electronic spin, $S \geq 1$, involve the zero-fielc splitting terms which are largely the result of spin-orbit coupling. In this section the relaxation mechanisms for ions with $S=1 / 2$ are discussed separately from those for which $S \geq 1$.

A. Mechanisms for $S=1 / 2$

1. Anisotropic $g$ and A Tensors

One of the most widely observed electron sp in relaxation mechanisms in liquids is the "microcrystalline tumbling" mechanism proposed by McConnell (1956). In McCornell's model the metal ion and its associated ligands were proposed to tumble as a whole. If the $g$ values 
and electron-nuclear hyperfine constant, $A$, of the complex were anisotropic, then tumbling of the complex would modulate the energy of the EPR transition and cause relaxation. What is most interesting about this mechanism is that it predicts relaxation rates which depend on the nuclear spin quantum number, $M_{I}$, of the transition being observed. Kivelson (1960) used the linear response theory of Kubo and Tomita (1954) to calculate the relaxation rates from this mechanism. He found that the relaxation from this mechanism could be divided into nonsecular terms, which contribute to both $T_{1}$ and $T_{2}$, and secular terms, which contribute only to $T_{2}$. There are also pseudosecular tenms, which directly contribute on? $y$ to $T_{2}$, but which affect the saturation behavior of the EPR transitions. Later, Wilson and Kiveison (1966a) repeated the calculation using a more accurate static Hamiltonian. They found

$$
\frac{1}{T_{2}}=\alpha+B M_{I}+r M_{I}^{2}+8 M_{I}^{3}
$$

where

$$
\begin{aligned}
\frac{a}{\tau_{R}}= & \frac{4}{45}\left(\Delta Y B_{0}\right)^{2}+\frac{3}{40} b^{2} I(I+1) \\
& -\frac{1}{30} b\left(\frac{a}{\omega_{0}}\right) \Delta Y B_{0} I(I+1)+u\left[\frac{1}{15}\left(\Delta \gamma_{0}\right)^{2}+\frac{7}{40} b^{2} I(I+1)\right. \\
& \left.-\frac{1}{30} b\left(\frac{a}{\omega_{0}}\right) \Delta Y B_{0} I(i+1)-\frac{5}{40} b^{2}\left(\frac{a}{\omega_{0}}\right) I(I+1) f\right]
\end{aligned}
$$


6

$$
\begin{aligned}
\frac{B}{\tau_{R}}= & \frac{4}{15} b \Delta \gamma B_{0}-\frac{8}{45}\left(\Delta \gamma B_{0}\right)^{2}\left(\frac{a}{\omega_{0}}\right) \\
& -b^{2}\left(\frac{a}{\omega_{0}}\right)\left[\frac{1}{20} I(1+1)+\frac{3}{40}\right]+u\left[\frac{1}{5} b \Delta \gamma B_{0}-\frac{2}{15}\left(\Delta \gamma B_{0}\right)^{2}\left(\frac{a}{\omega_{0}}\right)(1+f)\right] \\
= & \frac{1}{20} b^{2}\left(\frac{a}{\omega_{0}}\right) u[I(I+1)+7 I(1+1) f], \\
\frac{r}{\tau_{R}}= & \frac{1}{8} b^{2}-\frac{7}{30} b\left(\frac{a}{\omega_{0}}\right) \Delta \gamma B_{0} \\
& -u\left[\frac{1}{40} b^{2}+\frac{1}{6} b\left(\frac{a}{\omega_{0}}\right)_{\Delta \gamma B_{0}}+\left(\frac{2}{5} b \Delta \gamma B_{0}-\frac{5}{40}\right) b^{2} \quad\left(\frac{a}{\omega_{0}}\right) f\right] \\
\frac{\delta}{\tau_{R}}= & \frac{1}{20} b^{2}\left(\frac{a}{\omega_{0}}\right)+\frac{1}{20} b^{2}\left(\frac{a}{\omega_{0}}\right) u(1+f),
\end{aligned}
$$

where

$$
\begin{aligned}
& a=1 / 3\left(2 A_{1}+A_{\|}\right), \\
& b=2 / 3\left(A_{\|}-A_{1}\right), \\
& \Delta g=g_{\|}-g_{1}, \\
& \Delta r=\beta_{0} \Delta g / \hbar, \\
& B_{0}=\hbar \omega_{0} / g \beta_{0}, \\
& g=1 / 3\left(2 g_{1}+g_{\|}\right), \\
& u=1 /\left(1+\omega_{0}^{2} \tau_{R}^{2}\right), \\
& f=\omega_{0}^{2} \tau_{R}^{2} u u^{2},
\end{aligned}
$$


$\omega_{0}$ is $2 \pi$ times the observing frequency, and $B_{0}$ is the Bohr magnetron. The tumb?ing correlation time, $\tau_{R}$ is give.i by the Stokes-Einstein relation,

$$
\tau_{R}=\frac{4 \pi a^{3}}{3 k^{\top}} n,
$$

where $\eta$ is the viscosity of the liquid, $a$ is the hydrodynamic radius of the complex, $k$ is Boltzman's constant, and $T$ is the absolute temperature. The definition of the correlation time treats the complex as a tumbling sphere. This relaxation mechanism is strongly field dependent. Its contribution to $T_{1}$ is detailed in a later section.

2. Spin-Rrtation

The spin-rotition relaxation mechanism is due to the interaction of the spin magnetic moment with the rotational magnetic moment of the molecule. The rotational magnetic moment arises because the electrons do not follow the motion of the nuclei rigidly. A charge imbalance is created which generates a magnetic field as the molecule rotates. The energy of interaction of the magnetic field with the spin is given by

$$
\mathcal{K}_{\mathrm{SR}}=\underline{\mathrm{J}} \cdot \stackrel{\mathrm{C}}{\approx}, \underline{\mathrm{S}},
$$

where $\mathcal{J}$ is the rotational angular momentum, $\underset{\approx}{\mathcal{C}}$ is the spin-rotational coupling tensor, and $\underset{\sim}{S}$ is the spin c.ngular momentum. Relaxation can occur when $\mathcal{J}$ is modulated by collisions or $\underset{\approx}{C}$ is modulated by reorientation of the molecule. In liquids the modulation of $\underset{\sim}{J}$ is unually 
much faster than the modulation of $\underset{\approx}{C}$. Unoer these conditions, Hubbard (1963) found for molecules with cylindrical symmetry, in the extreme narrowing limit,

$$
\frac{1}{T_{2}}=\frac{1}{T_{1}}=\frac{2 I T \tau_{\omega}}{3 h^{2}}\left(2 C_{1}^{2}+C_{\|}^{2}\right),
$$

where $I$ is the molecular moment of inertia, $T$ is the absolute temperature, $C_{\|}$and $C_{1}$ are the components of $C$ along the molecular axis and perpendicular to it, respectively, and $\tau_{\omega}$ is the angular momentum correlation time. The components of $\mathrm{c}$ are seldom known, but Cur $\mathrm{l}$ (1965), and Atk ins and Kevelson (1966) were able to relate the spinrotation tensor to the $\mathrm{g}$ tensor. If use is made of hubbard's relaticn

$$
\tau_{\omega} T_{R}=I / 6 k T,
$$

a useful expression for the spin-rotational contribution to relaxation is

$$
\frac{1}{T_{1}}=\frac{1}{T_{2}}=\frac{1}{9 \tau_{R}}\left[\left(g_{\|}-g_{e}\right)^{2}+2\left(g_{1}-g_{e}\right)^{2}\right] \text {, }
$$

where $g_{e}$ is the free electron $g$ value. Equation (2.8) is the result of second-order perturbation theory and is most accurate for transition metal ions when the spin-orbit coupling is small compared to the crystal field splitting. Note that Eqs. (2.8) and (2.6) predict that 
for this mechanism, $\frac{1}{T_{1}}$ is proportional to $T / \pi$ and is independent of the magnetic field.

3. Electric Field Fluctuations

Kivelson (1966) consideled three reiaxation mechanisms for which the modulation of the intermolecular potentials by molecular motion contribute through the spin-orbit coupling to the electron spin relaxation. Two of these mechanisms were first proposed by Van Vleck (1540) to explain eiectron spin relaxation in crystals. The third is dise to Orbach $(1961)$.

The first mechanism Kivelson considered is called the Var Vleck-direct, because an electron spin is flipped by the direct interaction with a single phonon from the lattice. The rontribution to $T_{1}$ from this mechanism is

$$
\frac{1}{T_{1}}=\frac{1}{T_{2}}=64\left(\frac{\lambda}{\Delta}\right)^{2}\left(\frac{\phi q_{0}}{\Delta r_{0}}\right)^{2} \frac{\left(\omega_{0}{ }^{\top} c\right)^{2} \tau_{c}^{-1}}{1+\omega_{0}^{2} \tau_{c}^{2}}
$$

where $\lambda$ is the spin orbit coupling constant, $\Delta$ is the energy separation between the ground state and the first excited state connected to it by spin-orbit coupling, is the magnitude of the time dependent potential, $q_{0}$ is the root-mean-square amplitude of intermolecular oscillations, $r_{0}$ is the characteristic intemolecular distance, and $\tau_{c}$ is the correlation time.

The second process Kivelson considered is called the Van Vleck Raman process, because the spin is relaxed by a two-phonon process.

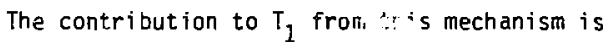




$$
\frac{1}{T_{1}}=32\left(\frac{\lambda}{\Delta}\right)^{2}\left(\frac{\phi a_{0}}{\Delta r_{0}}\right)^{4} \tau_{c}^{-1},
$$

when $\omega_{0}^{2} \tau_{c}^{2} \ll 1$. Kivelson concluded that both the Van vleck

direct and Raman processes are neglicible for liquids unless there is a low lying excited state connecsed to the ground state by spin-orbit coupling. However, if there is such a low lyirig state, relaxation can be very fast indeed.

The final process considered by Kivelson was the Orbach mechanism. In this mechenism an irteraction with a phonon from the ?attice causes a simultanenus electronic excitation and sp in flip. The contribution of this mechanism to the relaxation rate is

$$
\frac{1}{T_{1}}=16\left(\frac{\lambda}{\Delta}\right)^{2}\left(\frac{d \varepsilon_{0}}{\Delta r_{0}}\right)^{2}\left(\frac{\Delta}{\delta_{o n}}\right)^{2} \frac{\lambda^{-1} \tau_{c}^{-1}}{\left[\exp \left(\frac{1 \delta_{o n}}{k T}\right)-1\right]}
$$

where $\delta_{\text {on }}$ is the transition energy to the lowest orbitaliy excited state. This expression is correct only for $\delta_{\text {on }}^{2} \tau_{c}^{2} \leq 1$. Kivelson concludes that the Orisdch process could be important for liquids if on is sufficiently small.

The correlation time, $\tau_{c}$, for these three mechanisms should be characteristic of the translational fluctuations of the molecules in the liquid. Kivelson used $\tau_{c}=10^{-12} \mathrm{sec}$ in his estimation of the importance of these mechanisms. 
B. Mechanisms for $S \geq 1$

Transition metal ions with $S \geq 1$ have in their spin-Haniltonian a term which is given by

$$
\pi_{Z F S}=0\left[S_{z}^{2}-1 / 2 S(S+1)\right]+E\left(S_{x}^{2}-S_{y}^{2}\right),
$$

where $D$ and $E$ are the components of the zero-field-splitting (ZFS) tensor. Modulation of this term is a powerful relaxation mechanism for ions with $S \geq 1$. The term involving $E$ varishes for axial syrmetry, and the ZFS interaction vanishes completely for cubic symmetry. McGarvey (1957), and Carrington and Luckhurst (1964) considered relaxation by rotational modulation of the ZFS interaction, but the complexes studied in this work have cubic symmetry and should thus have a vanishing static ZFS. Bloembergen and Morgan (1961) suggested that dynamic fluctuations in the ZFS caused by distortions of the complex could be the cause of $s p$ in relaxation for cubic complexes with $S \geq 1$. Most calculations of the magnitude of the relaxation rates from the modulation of a dynamic ZFS have employed the relaxation theory of Redfield (1957). The use of this theory involves the calculation of the elements of the relaxation matrix $R$, which are given by

$$
\begin{aligned}
& R_{K K^{\prime} \lambda \lambda^{\prime}}=2 J_{K \lambda K^{\prime} \lambda^{\prime}}(K-\lambda)-\sum_{Y} \delta_{K^{\prime} \lambda^{\prime} J_{Y \lambda Y K}(Y-\lambda)}(Y-\lambda) \\
& -\frac{Y}{Y} \delta_{K \lambda} J_{Y \lambda^{\prime} Y K^{\prime}}\left(\gamma-\lambda^{3}\right),
\end{aligned}
$$


where the $J ' s$ are spectral densities, and $\delta$ is a Kronecker deita function. The form of the spectral densities depends on the nature of the perturbation and the type of motion involved in the relaxaticn Nic chanism.

Rubinstein, Barum, and Luz (1971) used the Redfield approach to calculate relaxation times from modulation of the ZFS. They assumed that the fluctuations only changed the direction of the principal components of the ZFS tensor, and not the ir magnitudes. In this work we are interested in ions with $S=3 / 2$ and $S=5 / 2$. For $S=3 / 2$, Rubinstein, Barum, and Luz found that there are two transverse relaxation times and two longitudinal relaxation times. The two transverse times are given by

$$
\frac{1}{2 e^{(I)}}=\frac{6}{5} \Delta^{2}[j(\omega)+j(2 \omega)]
$$

and

$$
\frac{1}{2 e^{(I I)}}=\frac{6}{5} \Delta^{2}[j(0)+j(2 \omega)]
$$

The longitulinal times are

$$
\frac{1}{1 e^{(I)}}=\frac{12}{5} \Delta^{2} j(u)
$$


and

$$
\frac{1}{1 e^{(I I)}}=\frac{12}{5} \Delta^{2} j(2 \omega)
$$

where,

$$
\Delta^{2}=\frac{2}{3} D^{2}+E^{2}
$$

and

$$
j(n \omega)=\frac{T}{1+n^{2} \omega^{2} T^{2}}
$$

These results are the same as those obtained by Carrington and Luckhurst (1964) for rotational modulation of a static ZFS, except that in this case

$$
\frac{1}{\tau}=\frac{1}{\tau_{R}}+\frac{n}{n-1} \frac{1}{\tau_{c}}
$$

where $\tau_{R}$ is the correlation time for rotational diffusion and $\tau_{c}$ is the correlation time for distortions of the complex along $n$ equivalent axes. The intensity of the EPR transition which is labeled $I$, is 1.5 times the intensity of the transition labeled $I I$.

For $S=5 / 2$, a closed form solution to the relaxation problem is not possible except when $\omega^{2} \tau^{2} \ll 1$. In that case 


$$
\frac{1}{T_{1 e}}=\frac{1}{T_{2 e}}=\frac{32}{5} \Delta^{2} \tau
$$

The correlation time, $\tau_{c}$, for the fluctuations responsible for the instantaneous disturtions of the complex should be in the same range as those for the electric field fluctuation mechanism for $S=$ $1 / 2$, about $10^{-12} \mathrm{sec}$. 
III. Theory of Dynamic Nuclear Polarization

A. DNP in Solution

The existence of dynamic nuclear polarization as a consequence of the relaxation processes in coupled systems of electron and nuclear spins was first predicted by Overhauser (1953). The theory and some early experimental results are discussed extensively by Abragam (1961). Some of the import ant theoretical and experimental aspects of DNP applied to liquids are reviewed by Hausser and Stehlik (1968). The portions of the theory necessary for understanding the experimental results of this work are discussed in this section.

Many of the results of DNP experiments $c$ an be understood by considering some simple kirictic models. A treatment of a two spin system was given by Solomon (1955). It will be useful to review this treatment in some detail.

The energy levels of a system of two spins of $s p$ in 1/2, 1abeled I and $S$, are shown schematically in Fig. 1, along with the possible transitions between them. The rates of change of the populations of the levels can be written down in a straightforward manner as

$$
\begin{aligned}
& \frac{d P}{d t}++=-\left(w_{1}+w_{1}^{\prime}+w_{2}\right) p_{++}+w_{1}^{\prime} p_{+-}+w_{1} p_{-+}+w_{2} p_{--}, \\
& \frac{d P}{d t}+-=-\left(w_{0}+w_{1}+w_{1}^{\prime}\right) p_{+-}+w_{0} p_{-+}+w_{1} p_{-}+w_{1}^{\prime} p_{++}, \\
& \frac{d P}{d t}-+=-\left(w_{0}+w_{1}+w_{1}^{\prime}\right) p_{-+}+w_{1} p_{++}+w_{0} p_{+-}+w_{1}^{\prime} p_{--}, \\
& \frac{d p}{d t}--=-\left(w_{1}+w_{1}^{\prime}+w_{2}\right) p_{--}+w_{2} p_{++}+w_{1} p_{+-}+w_{1}^{\prime} p_{-+},
\end{aligned}
$$




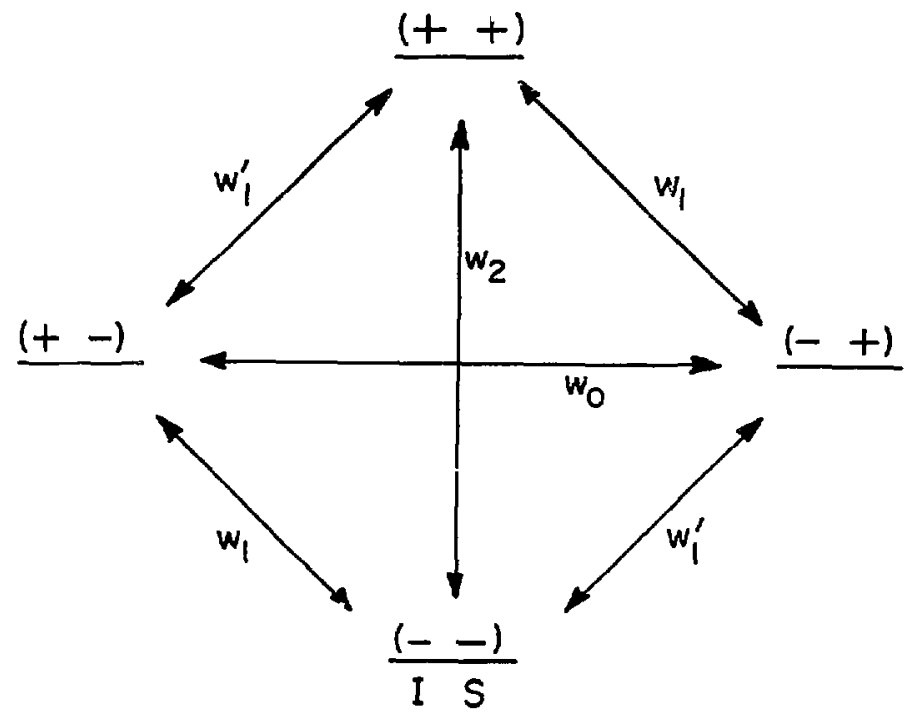

$X B L 827-5964$

Fig. 1. Relaxation in a system of two spins. 
where the P's are the populations of the respective levels and the w's are transitions between the levels, as designated in Fig. 1. The measurable quantities, the magnetizations $\left\langle S_{z}\right\rangle$ and $\left\langle I_{z}\right\rangle$ are related to the populations of the individual levels by

$$
K<I_{z}>=\left(P_{++}+P_{+-}\right)-\left(P_{-+}+P_{--}\right),
$$

and

$$
K<S_{z}>=\left(P_{++}+P_{-+}\right)-\left(P_{+-}+P_{--}\right),
$$

where $K$ is a proportionality constant. When combined with equations 3.1a-d, this definition yields the pair of coupled equation:

$$
\frac{d k I z}{d t}=-\left(w_{0}+2 w_{2}+w_{2}\right)\left(\left\langle I_{z}>-I_{0}\right)-\left(w_{2}-w_{0}\right)\left(\left\langle S_{z}\right\rangle-s_{0}\right)\right.
$$

and

$$
\frac{\left.d k S_{z}\right\rangle}{d t}=-\left(w_{2}-w_{0}\right)\left(\left\langle I_{z}\right\rangle-I_{0}\right)-\left(w_{0}-2 w_{1}^{1}+w_{2}\right)\left(\left\langle S_{z}\right\rangle-S_{0}\right) .
$$

Because interactions with the lattice tend to bring the system to thermal equilibrium, the quantities $\left\langle I_{z}\right\rangle$ and $\left\langle S_{z}\right\rangle$ are replaced by the differences from the ir respective equilibrium vaiues $I_{0}$ and $S_{0}$. If the $S$ spin is an electronic spin and the $I$ spin a nuclear spin, the system of equations is simplified. Mechanisms other than its interactions with the I spin are usually responsible for the rapid relaxation of the $S$ spin. Equation $3.2 b$ can thus be disregarded. Equation $3.2 \mathrm{a}$ solved in steady state gives 


$$
\left(\left\langle I_{z}\right\rangle-I_{0}\right)=-\frac{\left(w_{2}-w_{0}\right)\left(\left\langle S_{z}\right\rangle-S_{0}\right)}{\left(w_{0}+2 w_{1}+w_{2}\right)}=-\frac{0}{0}\left(\left\langle S_{z}\right\rangle-S_{0}\right),
$$

where $0=\left(w_{2}-w_{0}\right)$ and $o=\left(w_{0}+2 w_{1}+w_{2}\right)$. The specific enhancement, E can the defined

$$
E \equiv 1-\frac{\left\langle I_{2}\right\rangle}{I_{0}},
$$

so

$$
E=\frac{\sigma\left(\left\langle S_{z}\right\rangle-S_{0}\right)}{\rho I_{0}}
$$

Saturation of the S spin would lead to

$$
E=-\frac{o S_{0}}{\rho I_{0}}
$$

A slightly more complicated situation arises when the electronic spin is coupled to a third spin, $K$, resulting in hyperfine splitting of the EPR spectrum. The number of levels to consider in a kinetic model grows from 4 to $4 \times(2 K+1)$. However, transitions which involve a change of the state of the $K \mathrm{sp}$ in do not contribute to the relaxation of $I$, because we assume that there is no direct coupling between the $I$ and $K$ spin. This makes it possible to treat this system as $2 K+1$ separate two spin systems, each with equations of motion 


$$
\frac{d<I z\rangle_{m}}{d t}=-o_{m}\left(\left\langle I_{z}\right\rangle_{m}-I_{o m}\right)-\sigma_{m}\left(\left\langle S_{z}\right\rangle_{m}-S_{o m}\right),
$$

where $m=M_{K}$. Only the total $I_{z}=\sum_{m}\left\langle I_{z}\right\rangle_{m}$ is measurable, however, so

$$
\frac{d\left\langle I_{z}\right\rangle}{d t}=\sum_{m=-K}^{K}-o_{m}\left(\left\langle I_{z}\right\rangle-I_{o m}\right)-\sigma_{m}\left(\left\langle S_{z}\right\rangle_{m}-S_{o m}\right)
$$

When hyperf ine lines are well resolved, it is usually possible to get appreciable saturation in only one line. If this is the case, only one of the $2 K+1$ systems is affected. The rest remain at their equilibrium values. When the $m^{\prime}$ th $l$ ine is saturated, in steady state, Eq. (3.7) becomes

$$
<\mathrm{I}_{z}>_{\mathrm{m}}-\mathrm{I}_{\mathrm{om}}=\frac{\sigma_{\mathrm{m}}}{\mathrm{o}_{\mathrm{m}}} \mathrm{s}_{\mathrm{on}}
$$

The observable $\left\langle I_{z}\right\rangle=\left\langle I_{z}\right\rangle_{m}+\sum_{n \neq m} I_{\text {on }}$, so

$$
\left\langle I_{z}\right\rangle-I_{0}=\frac{\sigma_{m}}{\rho_{m}} S_{a m}
$$

Since $S_{0 m} \approx S_{0} /(2 K+1)$, the amount of enhancement observable will be decreased by a factor of $2 K+1$.

If the hyperf ine 1 ines are not well resalved, Eq. (3.7) can only be collapsed under certain circumstances. If the hyperfine splitting constant is small compared to the static field, all of the $\rho_{m}$ 's can be considered to be approximately equal. Then 


$$
E=\frac{-1}{\rho I_{0}} \sum_{m=-K}^{K} a_{m}\left(\left\langle S_{z}\right\rangle_{m}-S_{o m}\right),
$$

where $p^{\prime}=\rho_{-K}=p_{-K}+1 \cdots=p_{K}$.

The use of kinetic models to describe DNP becomes increasingly difficult as one tries to apply them to systems with I or S greater than one-half. Also one eventually has to be more specific about what the rates $w_{0}, w_{1}$, and $w_{2}$ actually are in order to properly evaluate the frequency and temperature dependence of the DNP.

The semiclassical density matrix formulation of Abragam (1961) can be used to calculate the relaxation rates of a sp in subject $: 0$ a time dependent Hamiltonian, $\mathcal{H}^{\prime}(t)$. For DNP

$$
\Psi^{\prime}(t)=A \vec{I} \cdot \vec{S}+\frac{3(\vec{I} \cdot \vec{r}\rangle\langle\vec{S} \cdot \vec{r}\rangle}{r^{5}}-\frac{\vec{I} \cdot \vec{S}}{r^{3}} ;
$$

where $\vec{r}$ is the distance vector between the two spins. $\vec{I}$ and $\vec{S}$ are the nuclear and electronic spin operators respectively. The first term is the Fenni-contact interaction between the nucleus and the electron. The second two terms are the electron-nuclear dipolar interaction. The dipolar interaction can be rewritten as

$$
W^{\prime}\left(\text { dipolar) }=\sum_{q=-2}^{2} F^{(q)} A^{(q)},\right.
$$

where 
21

$$
\begin{aligned}
& F^{(1)}=\sin \theta \cos \theta e^{i \phi} / r^{3}, \\
& F^{(2)}=\sin ^{2} \theta e^{2 i \phi} / r^{3}, \\
& F^{(0)}=1-3 \cos ^{2} \theta / r^{3},
\end{aligned}
$$

and the angles $\theta$ and give the orientation: of $r$ with respect to the static magnetic field. For $A^{(q)}$ we have,

$$
\begin{aligned}
& A^{(0)}=a\left[-\frac{2}{3} I_{z} S_{z}+\frac{1}{6}\left(I_{+} S_{-}+I_{-} S_{+}\right)\right] \\
& A^{(1)}=a\left[I_{z} S_{+}+S_{z} I_{+}\right], \\
& A^{(2)}=\frac{a}{2}\left[I_{+} S_{+}\right],
\end{aligned}
$$

where

$$
\begin{aligned}
& a=-\frac{3}{2} \gamma_{I} Y_{S}, \\
& S_{+}=S_{x}+i S_{y}, I_{+}=I_{x}+i S_{y}, \\
& S_{-}=S_{x}-i S_{y}, I_{-}=I_{x}-i S_{y},
\end{aligned}
$$

and,

$$
\begin{aligned}
& F^{(q)}=F^{-q^{*}} \\
& A^{-(q)}=A^{q}
\end{aligned}
$$


The time dependence comes either from variatior:s of $r$ or $\theta$ and $b$ from translation or rotation in the system containing $I$ and $S$, or from modulation of the $S$ spin due to electron spin relaxation. The time dependence of the scalar interaction, AS-I can come from modulation of A by chemical exchange or from modulation of $S$ from electron spin relaxation.

Using the semiclassical densily matrix approach, Abragam finds

$$
\frac{d\left\langle I I_{z}\right\rangle}{d t}=-p\left[\left\langle I_{z}\right\rangle-I_{0}\right]-\sigma\left[\left\langle S_{z}\right\rangle-S_{0}\right],
$$

where

$$
\rho=\frac{r_{I}^{2} r_{s}^{2}}{10 r^{6}}\left(6 J\left(\omega_{I}+\omega_{s}\right)+J\left(\omega_{I}-\omega_{s}\right)+3 J\left(\omega_{l}\right)\right) \text {, }
$$

and

$$
0=\left(6 J\left(\omega_{I}+\omega_{S}\right)-J\left(\omega_{I}-\omega_{S}\right)\right)
$$

Note that this is the same form as obtained by the use of the kinetic models. In this case $\sigma$ and $o$ are given in tienms of the spectral density functions $J_{q}(\omega)$, where

$$
\left.J_{q}(\omega)=\int_{-\infty}^{\infty} \overline{\left\langle F^{q}(t) F^{q}(t+\tau)\right.}\right\rangle e^{-i \omega t} d t
$$


and

$$
\left\langle F^{(q)}(t) F^{(q)}(t+\tau)\right\rangle
$$

is the autocorrelation, function of the random force $F^{(q)}$. Equation (3.12) is exact for both spins 1/2, and is correct for spins greater than $1 / 2$ within the accuracy of the high temperature approi.tiration (Abragam, 1961, D. 201).

The experimental work discussed in later chapters concerns the DNP of solvent molecules in a solution of paramagnetic ions or molecules. The paramagnetic species often farm complexes with the solvent that have an average lifetime, $\tau_{h}$, which is longer than the correlation time for the interaction responsible for the relaxation of the nuclear spin. If this is the case, a treatment of the motion as classical rotational diffusion leads to

$$
\begin{aligned}
& 0=\frac{r_{I}^{2} Y_{S}^{2} S(S+1)}{15 r^{6}}\left[6 \tau_{c}+\frac{14 \tau_{c}}{1+\omega_{e}^{2} \tau_{c}^{2}}\right]+\frac{2 S(S+1) A^{2}}{3}\left[\frac{{ }^{\tau} x}{1+\omega_{e}^{2} \tau_{x}^{2}}\right], \\
& 0=\frac{I(I+1){ }^{2} I^{2} \gamma_{S}^{2}}{15 r^{6}}\left[\frac{10 \tau_{c}}{1+\omega_{e}^{2} 2}\right]-\frac{2 I(I+1) A^{2}}{3}\left[\frac{{ }^{\tau} x}{1+\omega_{e}^{2} \tau_{x}^{2}}\right],
\end{aligned}
$$

where

$$
\begin{aligned}
& \frac{1}{\tau_{c}}=\frac{1}{\tau_{h}}+\frac{1}{\tau_{R}}+\frac{1}{T_{1 \mathrm{e}}}, \\
& \frac{1}{\tau_{x}}=\frac{1}{\tau_{h}}+\frac{1}{T_{1 e}},
\end{aligned}
$$


and $\tau_{R}$ is correlation time for rotational diffusion and $T_{1 e}$ is the electron spin relaxation time. We have assumed that $\omega_{I} \ll \omega_{S}$ and ${ }^{\omega_{I}} \tau_{c}^{2} \ll 1$. For the ions studied in this work $T_{l e} \geq 10^{-10} \mathrm{sec}$ and $\tau_{h} \geq 10^{-10} \mathrm{sec}$. At the magnetic field used for these experiments $\omega_{e}^{2} \tau_{x}^{2} \gg 1$, making the effect of scalar coupling small compared to

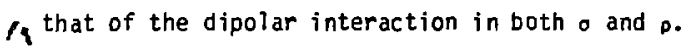

There is evidence for some radicals and paramagnetic ions that long-lived complexes are not formed (Pfeifer, 1961, Güenter and Pfeifer, 1964). In this case DNP is governed by the translational diffusion of the paramagnetic species. For translation the calculation of the spectral densities is more complicated. Abragam finds

$$
\frac{\sigma}{\rho}=\frac{6 J\left(\omega_{I}+\omega_{s}\right)-J\left(\omega_{I}-\omega_{s}\right)}{6 J\left(\omega_{i}+\omega_{S}\right)+3 J\left(\omega_{I}\right)+J\left(\omega_{I}-\omega_{s}\right) S(S+I)},
$$

where for pure translational motion

$$
J(\omega)=\frac{\tau_{t} I(x)}{d^{3}},
$$

and

$$
I(x)=x^{-5}\left\{x^{2}-2+e^{-x}\left[\left(x^{2}-2\right) \sin x-\left(x^{2}+4 x+2\right) \cos x\right]\right\},
$$

with 


$$
x=\left(2 \omega_{S}{ }^{T}\right)^{1 / 2}
$$

and

$$
\tau_{t}=\frac{d^{2}}{D_{l}+D_{S}}
$$

$\mathrm{D}_{\mathrm{I}}$ and $\mathrm{D}_{\mathrm{S}}$ are the translational diffusion coefficients of the molecules containing the nuclear and electronic spins respectively, and $d$ is the distance of closest approach of the two molecules.

The dependence of the quotient o/p on frequency and temperature for translational motion is somewhat different from rotational motion, particulariy in the region where $\omega_{\mathrm{e}}^{2} \mathrm{x}^{2} \geq 1$. Both functions approach zero as $\omega^{2} \mathrm{e}^{2}$ gets large, but in the translational case the approach is much more gradual. As $\omega_{\mathrm{e}^{\mathrm{T}}}^{2}$ gets small both functions approach $\frac{1}{2(I) S+1)}$. For small $\omega_{e^{2}}^{2}$,

$$
E=-\frac{1}{2} \frac{S_{0}}{I_{0}} \approx 330
$$

This means that the NMR signal when the EPR line is saturated is roughly 330 times as intense and in the apposite direction of the NMR signal without DNP. If the electron experiences hyperf ine coupling from another nucleus of spin $k$, this enhancement is reduced by a ractor of $2 k+1$, as was explained by the kinetic model. This is because electrons which are not affected by the saturating fielo because they are out of resonance still contribute to the relaxation of the spin I. 
The enhancement will also be reduced from its theoretical maximum by the existence of any relaxation mechanism for the nucleor spin other than interaction with the electron spin. An extra term must be added to $\rho$ to account for this independent relaxation; it is usually refered to as the "leakage" term. In solutions of paramagnetic ions it is usually possible to keep the leakage term relatively small by making the solution sufficiently concentrated in the paramagnetic species. B. DNP in the Presence of Chemical Exchange

The model previously discussed only treats a pair of isolated interacting spins. In a dilute solution of paramagnetic ions only a small fraction of the nuclei are in close $c$, itact with the paramagnetic ion at any particular instant. If DNP is to be observed in the bulk nuclei there must be chemical exchange between the paramagnetic complex and the bulk.

McConnell (1958) gives modified Bloch (1946) equations for two site chemical exchange. The equations can be modified further to include steady state polarization of the minority species. For the $z$ components of the magnetization one has

$$
\begin{aligned}
& \frac{d M_{z}^{A}}{d t}=\frac{M_{0}^{A}}{T_{1 A}}-\frac{M_{z}^{A}}{T_{1 A}}-\frac{M_{z}^{A}}{T_{A}}+\frac{M_{z}^{m}}{\tau_{m}^{m}}, \\
& \frac{d M_{z}^{m}}{d t}=\frac{M_{0}^{m}}{T_{1 m}}+\frac{\delta M}{T_{1 m}}-\frac{M_{z}^{m}}{T_{1 m}^{m}}-\frac{M_{z}^{m}}{T_{m}^{m}}+\frac{M_{z}^{A}}{T_{A}},
\end{aligned}
$$


where

$$
\begin{aligned}
& M_{z}^{A}=\text { bulk magnetization, } \\
& M_{Z}^{m}=\text { magnetization for paramagnetic complex, } \\
& T_{1 A}=\text { spin lattice relaxation time in the b.lk, } \\
& T_{1 m}=\text { spin lattice relaxation time on the complex, } \\
& \tau_{A}=\text { lifetime in the bulk, } \\
& \tau_{m}=1 \text { ifetime in the paramagnetic complex, } \\
& \delta M=\text { the effect of DNp pulling the m spins away from equilibrium. }
\end{aligned}
$$

The modified Bloch equations have been solved in steady state with an r.f. field present (Swift and Connick, 1962) and as differential equations (leigh, 1971) to detemine the apparent relaxation rates of the bulk magnetization, but these calculations have not been extended to detemine the magnetization itself. The system of Eqs. (3.19a) and (3.19b) can be manipulated to yield a single second order differential equation,

$$
\tau_{m} \frac{d^{2} M_{z}^{A}}{d t^{2}}+\left(\frac{\tau_{m}}{\tau_{1 m}}+\frac{\tau_{m}}{\tau_{1 A}}\right) \frac{d M_{z}^{A}}{d t}+\left(\frac{\tau_{m}}{\tau_{1 m}{ }^{\tau_{1 A}}}-\frac{1}{\tau_{A}}\right) M_{z}^{A}=\frac{M_{0}^{m}+\delta M}{\tau_{1 n}}+\frac{\tau_{m} M_{0}^{A}}{\tau_{1 m} T_{1 A}}
$$

where using McConnell's notatian,

$$
\frac{1}{\tau_{1 A}}=\frac{1}{T_{1 A}}+\frac{1}{\tau_{A}}
$$


and

$$
\frac{1}{\tau_{1 m}}=\frac{1}{1 m}+\frac{1}{\tau_{m}}
$$

At $\mathbf{t}=0$ both magnetizations are at their equilitrium values. The electron spins are then irradiated, activating the $\delta H / T_{1 m}$ term. The magnetizations are allowed to propagate according to Eq. (3.20). Under these conditions the solution to Eq. $(3.20)$ is

$$
M_{z}^{A}=c_{+} e^{r_{+} t}+c_{-} e^{r_{-} t}+m_{\infty},
$$

where $r_{ \pm}$is the same as determined by Leigh,

$2 \tau_{m} r_{ \pm}=-\frac{\tau_{m}}{\tau_{1 m}}+\frac{{ }^{\tau} m}{\tau_{1 A}} \pm \sqrt{\left(\frac{\tau_{m}}{\tau_{1 m}}+\frac{{ }^{\tau} m}{\tau_{1 A}}\right)^{2}-4 \tau_{m}\left(\frac{\tau_{m}}{\tau_{1 A}{ }^{\tau} 1 m}-\frac{1}{\tau_{A}}\right)}$, and $m_{\infty}$ is the solution as $t$ approaches infinity, the steady state solution.

We have for the steady state solution,

$$
M_{\infty}=\frac{\frac{M_{0}^{m}+\delta M}{T_{1 m}}+\frac{\tau_{m}^{m_{0}^{A}}}{{ }^{T_{1 m} T_{1 A}}}}{\frac{T_{m}}{{ }^{T_{1 m}{ }^{\top} 1 A}}-\frac{1}{{ }^{T} A}}
$$

The coefficients are given by 


$$
c_{+}=\frac{\left(M_{0}^{A}-M_{\infty}\right) r_{-}}{r_{-}-r_{+}}
$$

and

$$
c_{-}=\frac{\left(M_{0}^{A}-M_{\infty}\right) r_{+}}{r_{-}-r_{+}}
$$

In a dilute solution

$$
\frac{1}{f} \equiv \frac{M_{0}^{A}}{M_{0}^{m}}=\frac{\tau_{A}}{\tau_{m}} \gg 1 .
$$

Al so usually we have $f T_{1 A} \gg T_{1 m^{*}}$ With these approximations Leigh finds

$$
r_{+}=-\frac{f}{\left.T_{1 m}+T_{m}\right)}=-\frac{1}{T_{1 p}}
$$

This result was also found by Luz and Meiboom (1964) under slightly different approximations. Within the same degree of approximation

$$
r_{-}=-\left(\frac{1}{\tau_{m}}+\frac{1}{T_{1 m}}\right)
$$


With these approximations and the additional assumption of fast exch ange $\left(\tau_{m} \ll T_{1 m}\right)$

$$
M_{2}^{A}(t)=\left(M_{0}^{A}-M\right) e^{-t / T} 1 p+M_{\infty},
$$

and

$$
M_{s}=\frac{1}{f}\left(M_{0}^{m}+\delta M\right)
$$

Equations (3.27) and (3.28) show that the steady state magnetization of the bulk species does reflect the magnetization of the paramagnetic complex under the previously stated conditions. If exchange is not fast, the bulk magnetization will not accurately reflect the polarization in the complex. The polarization of the complex will also not be accurately reflected in the bulk if $T_{1 m}$ is not much smaller than $\mathrm{fT}_{1 A}$, even if exchange is fast. Corrections must be made to the observed polarization in cases of intermediate exchange very and dilute solutions. The only ion studied in this work for which slow exchange might be a problem is the $v 0^{2+}$ ion. The application of Eq. (3.22) using parameters from the work of wüthrich and Connick (1968) shows that the bulk polarization accurately reflects the polarization of protons bound to the metal ion for $0.05 \mathrm{M} \mathrm{vo}^{2+}$ solution, but that a $0.005 \mathrm{M}$ solution would result in inaccuracies. C. Measuring Spin Lattice Relaxation Times

The classical method for measuring spin-lattice relaxation times is to measure the absorption spectrum with increasing amounts of incident power. If the system obeys the 81 och (1946) equations, then 
when the absorption reaches its maximum as a function of power, $H_{1}^{2} \gamma^{2} T_{1} T_{2}=1$, where $H_{1}$ is the magnitude of the irradiating field, $r$ is the gyromagnetic ratio of the spin and $T_{2}$ is the spin-spin relaxation sime. This is often referred to as "half saturating" the signal since $s$, the saturation parameter, which is defined as

$$
\mathbf{s}=1\left(1+r^{2} H_{1}^{2} T_{1} T_{2}\right),
$$

equals 1/2. In EPR spectroscopy the first derivativa of the absorption is usually measured. When the first derivative is maximum, $s=2 / 3$. $\mathrm{T}_{2}$ and $\gamma$ can be determined from the low fower spectrum. $\mathrm{H}_{1}^{2}$ is proportional to the incident power and is in principle calculable, but this proves difficult in practice. Usually a sample of known $T_{1}$ must be used to determine $H_{1}^{2}$ for a given experimental arrangement.

The direct saturation method becomes difficult for the broad lines common to transition metal ions. A line $10 \mathrm{G}$ wide has $\mathrm{T}_{2}=6.5 \times$ $10^{-9} \mathrm{sec}$. If $T_{1}=T_{2}$, then to half saturate the 1 ine requires $H_{1}^{2}=75 \mathrm{G}^{2}$. Microwave fields of this size are beyond the range of conventional EPR spectrometers using conventional cavities and $k l y$ strons. Microwave sources with a larger power output than small klystrons, such as traveling wave tubes and magnetrons, are available, but the detection of absorption signals with these high puwer scurces is difficult due to the inherent noisiness of the high power sources and the lack of sensitive detectors which can operate in a high power environment (McCain, 1966). Furthenmore, many interesting EPR systems 
have linewidths considerably larger than $10 \mathrm{G}$. Since the power necessary to half saturate a line goes up roughly with the square of the linewidth, if $T_{1}=T_{2}$, power requirements escalate rapidly.

It is of course not necessary to half saturate a line to measure its $T_{1}$. Since

$$
\frac{1}{S}=1+\gamma^{2} H_{1}^{2} T_{1} T_{2}=\underset{\substack{H_{1}>0}}{\left[1 j m\left(v^{\prime} / H_{1}\right) /\left(v^{\prime} / H_{1}\right)\right]^{2 / 3}}
$$

where $v^{\prime}$ is the peak to peak amplitude of the derivative of absorption (Poole and Farrach, 1971), a plot of the right hand side of Eq. (3.30) vs $H_{1}^{2}$ would have a slope $r^{2} T_{1} T_{2}$. For transition metal ions the power necessary to make the ratio

$$
\left(\lim _{H_{1} \rightarrow 0} v^{\prime} / H_{1}\right) /\left(v^{\cdot} / H_{1}\right)
$$

measurably different from unity is larger than that available from conventional spectrometers, so this approach would suffer from the previously discussed problems with a high power spectrometer.

Dynamic Nuclear Polarization can be used to detemine the saturation parameter. If the electronic sp in obeys the Bloch equations, at resonance

$$
\left\langle S_{z}\right\rangle=\frac{S_{0}}{1+\gamma_{e}^{2} H_{1}^{2} T_{1 e} T_{2 e}}
$$


Substitution of this relation into Eq. (3.5) yields

$$
E=-\frac{\sigma S_{0} \gamma_{e}^{2} H_{1}^{2} T_{1 e} T_{2 e}}{\rho I_{0}\left(1+{ }^{2}{ }_{e} H_{1}^{2} T_{1 e} T_{2 e}\right)}
$$

A plot of $1 / \mathrm{E}$ vs $1 / \mathrm{H}_{1}^{2}$ would be linear with a slope of $1 / r_{e}^{2} \mathrm{~T}_{1} \mathrm{e}^{\mathrm{T}}$ ?e (Haupt and Muller-Warmuth, 1962; Richards and White, 1964). DNP has been used to measure electron $T_{1}{ }$ 's at 156 (Muller-Warmuth, 1963), and to measure the electron $T_{1}$ of free radicals at $3300 \mathrm{G}$ (Kramer, Muller-Warmuth, and Schindler, 1965).

McCa in and Myers $(1967,1974)$ used DNP to measure $T_{1 e}$ of transition metal ions. Because of the short relaxation times involved and the limited power of the ir spectrometer they found $H_{1}^{2} \gamma^{2} \mathrm{~T}_{1} \mathrm{~T}_{2} \ll 1$. This condition simplifies Eq. (3.32) to

$$
E=-\frac{\sigma S_{0} \gamma_{e}^{2} H_{1}^{2} T_{1 e} T_{2 e}}{\rho I_{0}},
$$

where $H_{1}^{2}$ is proportional to the incident power, $P$, and $I_{D}$ is proportional to the static field, H. Taking into account the possibility of being of $f$-resonance, and the additional complication of hyperfine structure yields

$$
E \propto \frac{p}{p H} \sum_{m} \frac{a_{m}^{T} 1 e m^{\top} 2 e m}{1+\left(\omega-\omega_{e m}\right) T_{2 e}^{2}} \text {. }
$$


Because the ratio $S_{0} / I_{0}$ is large (- 660 in the case of protons), a small and most likely immeasurable saturation of the electron spins of a few hundredths of a percent results in an enhancement of a few percent or more, which is easily measured. Equation $3.33 \mathrm{can}$ be used to obtain the relative $T_{1 e}$ of two species, where one of the species is a standard with a known $T_{l e}$.

The comparison of enhancements of different paramagnetic species is complicated because ofo is in general different for different species. The differences are particularly pronounced at the microwave frequency used in this work. A plot of ofo vs. $T$ at $X$-band $(9 \mathrm{GHz})$ is shown in Fig. 2. This plot assumes rotational modulation of the dipolar interaction as the interaction responsible for ONP. Some species will have DN determined by translational modulation. Fortunately the behavior of o/o can usually be detenined from measurement of nuclear magnetic relaxation times of the solvent. Hauser and Stehlik (1968) discuss a method for doing this, but of ten it is more convenient to fit the nuclear relaxation to a diffusional model and use the correlation times determined from nuclear relaxation measurements in Eqns. (3.13) and (3.14) or (3.16). This is often necessary because nuclear relaxation measurements are not always available at all of the frequencies and temperatures desired. 


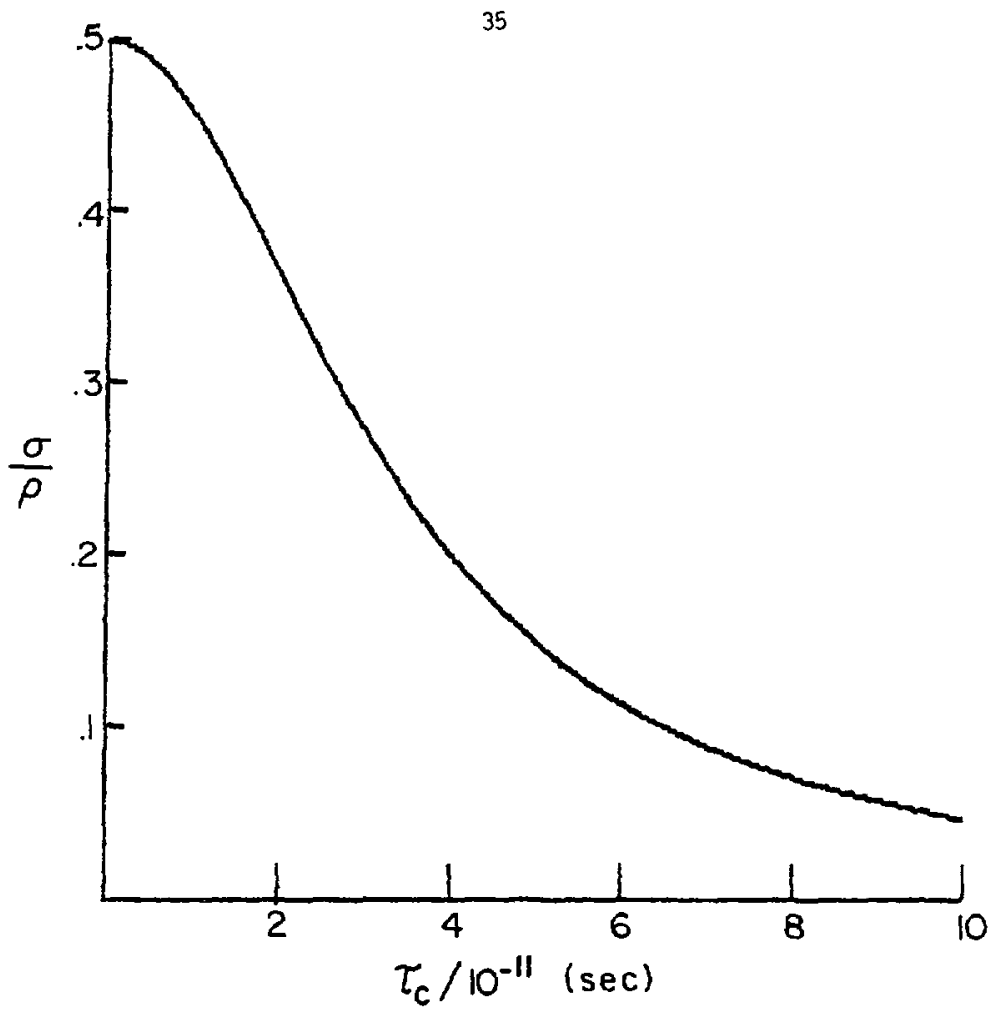

XBL $827-5965$

Fig. 2. o/p calculated from tive dipolar parts of equations 3.13 and 3.14 for $\omega_{e}=5.56 \times 1010 \mathrm{sec}^{-1}$ 
IV. Flow Spectrometer

A. Introduction

A major 1 imitation to the observation of DNP in aqueous soiutions is the large dielectric loss of water at micruwave frequencies. This limitation is particularly severe for transition metal ion solutions. Because of the ir short relaxation times, the level of microwave power. necessary to cause a measurable amount of DNP woulc also cause severe dielectric heating of the solution.

Another problem inherent in the observation of DNP is the inconvenience of irradiating the sample at both the EPR and MMR frequencies. The simplest experimental configuration, which would consist of simply placing the MR coil within the microwave cavity is unsatisfactory because the presence of the coil in the microwave fieid will both lirer the $Q$ of the cavity and shield the sample from the field, resulting in inefficient use of the saturating power. Several solutions to this problem are discussed by Hausser and Stehlik (1968).

McCa in and Myers (1967) solved both the dielectric heating and the coil gecmetry problems by placing the Mat coil just outside the cavity and flowing the solution through the cavity and then into the NMR coil. They were able to observe the DNP of transition metal ions at S-band $(3 \mathrm{GHz})$ without excessive heating of the solutions. An $x$-band $(9 \mathrm{GHz})$ spectrometer based on the same principle is described in the next section.

The use of the flow system adds additional complications to the interpretation of the measured enhancements. Because the solution is 
exposed to the microwave field for a limited time and because it takes time for the solution to reach the MMR coil, the observed enhancements will depend on the spin lattice relaxation time of the nucleus being observed. Also, the microwave field is in general not uniform along the flow path through the cavity.

One can model the build-up of enhancement by combining the definition of enhancement with the Bloch (1946) equations for the $z$ component of the magnetization. We get

$$
\frac{d E}{d t}=-\frac{E-E_{S S}}{T_{1}},
$$

where $E_{S S}$ is the steady-state value of the enhancement. Because the microwave field is non-uniform within the cavity we find that

$$
E_{s S}=k H_{1}^{2}(z)=k H_{1}^{2}(v t) \text {, }
$$

where $v$ is the flow velocity, $t$ is the time spent in the cavity and $k$ is determined from Eq. (3.33). For a narrow sample tube placed along the axis of a cylindrical cavity only the field components in the divection of the cavity axis contribute significantly to $\mathrm{H}_{1}^{2}$ over ine sample. For the $\mathrm{TE}_{011}$, mode

$$
H_{z}=H_{\max } \sin \left(\frac{\pi z}{d}\right),
$$

where $d$ is the length of the cavity (Poole, 1967). For this mode 
$H_{1}^{2}(v t)=H_{\max }^{2} \sin ^{2}\left(\frac{\pi v t}{d}\right)$

The solution of Eq. (4.1) yields

$$
E=\frac{k H_{\max }^{2}\left(1-e^{-\delta /\left(v T_{1}\right)}\right)}{2+\frac{1}{2}\left(\frac{d}{\pi v}\right)^{2} / T_{1}^{2}}
$$

for solution emerging from the cavity. For this field distribution the mean square field over the sample, $\left\langle H_{1}^{2}\right)$, is just half of the maximum field. Defining

$$
E_{A V} \equiv k\left(H_{1}^{2}\right)=\frac{k H_{\text {max }}^{2}}{2},
$$

we have

$$
E\left(\frac{d}{v}\right)=\frac{E_{A V}\left(1-e^{-d / v T_{1}}\right)}{1+\left(\frac{d}{2 \pi v}\right)^{2} / T_{1}^{2}} .
$$

Once the solution leaves the cavity the enhancement is expected to decay to zero exponentially, yielding

$$
E(v, d, a)=\frac{E_{A V}\left(1-e^{\left.-d / v T_{1 n}\right) e^{\left(-a / v T_{l n}\right)}}\right.}{1+\left(\frac{d}{2 \pi v}\right)^{2} / T_{I n}^{2}}
$$


where a is the distance from the cavity to the coil. This expression is plotted as a function of flow velocity in Fig. 3. Note that flowing the solution rapidly samples the average value of the microwave field, and loses a factor of two in enhancement compared to a static sample located at the maximum of the microwave field. The plot in Fig. 3 can be considered as $E$ vs. $v$ for constant $T_{1 n}$ or $E$ vs. $T_{\text {In }}$ for constant $v$. It is obvious that in order to compare the enhancements of two different solutions they should have roughly the same $T_{1 n}$. However, if one operates on the gently sloping portion of the curve small differences in $T_{\text {In }}$ will not have a large effect on the rela$t$ ive enhancements. The plot of measured enhancement versus flow rate in Fig. 4 shows qualitative agreement with this model. The points at the lowest rates probably show too much enhancement because the heating of the solution at those rates was not negligible.

\section{B. Spectrometer}

A simplified block diagram of the flowing sample DNP spectrometer is shown in Fig. 5. The solution being studied is pumped through Tygon tubing to a $1 \mathrm{~mm} i . d$. quartz tube which passes through the axis of the cynlindrical microwave cavity. Also in the flow path are thermometers to measure the temperature of the solution before and after $i t$ passes through the cavity, a flowmeter to measure the flow rate, and a heat exchanger which $c$ an be immersed in a water bath to control the temperature. The solution is pumped through the flow path with a magneíically coupled gear jump, the Paragon model 3316-2. A typical flow rate is $150 \mathrm{cc} / \mathrm{min}$. At this flow rate the temperature 


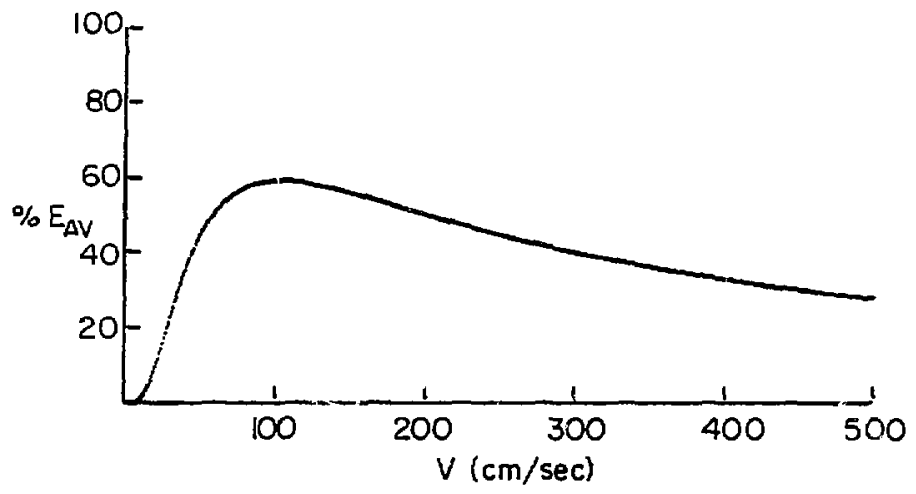

XBL 827-5966

Fig. 3. A plot of Eq. 4.7 vs flow velocity with $d=7 \mathrm{~cm}$, $a=3 \mathrm{~cm}$, and $T_{1 \mathrm{n}}=40 \mathrm{msec}$. 


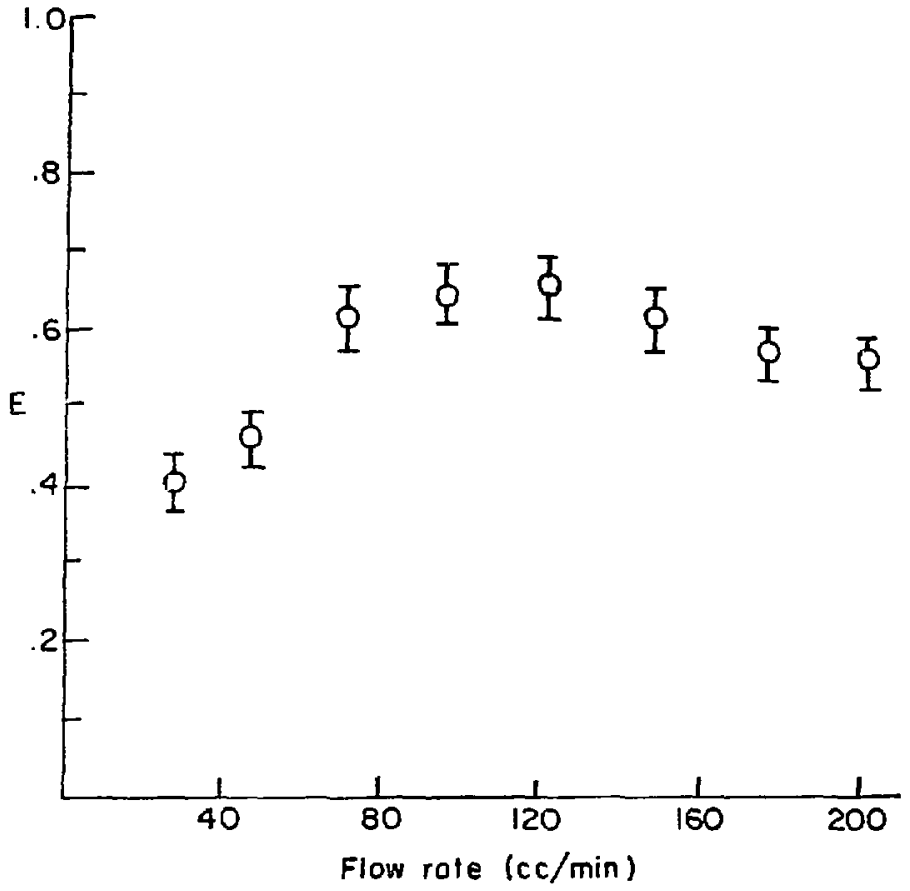

XBL B27-5967

Fig. 4. Enhancement vs flow rate for $0.031 \mathrm{M} \mathrm{voSO}_{4}$. 


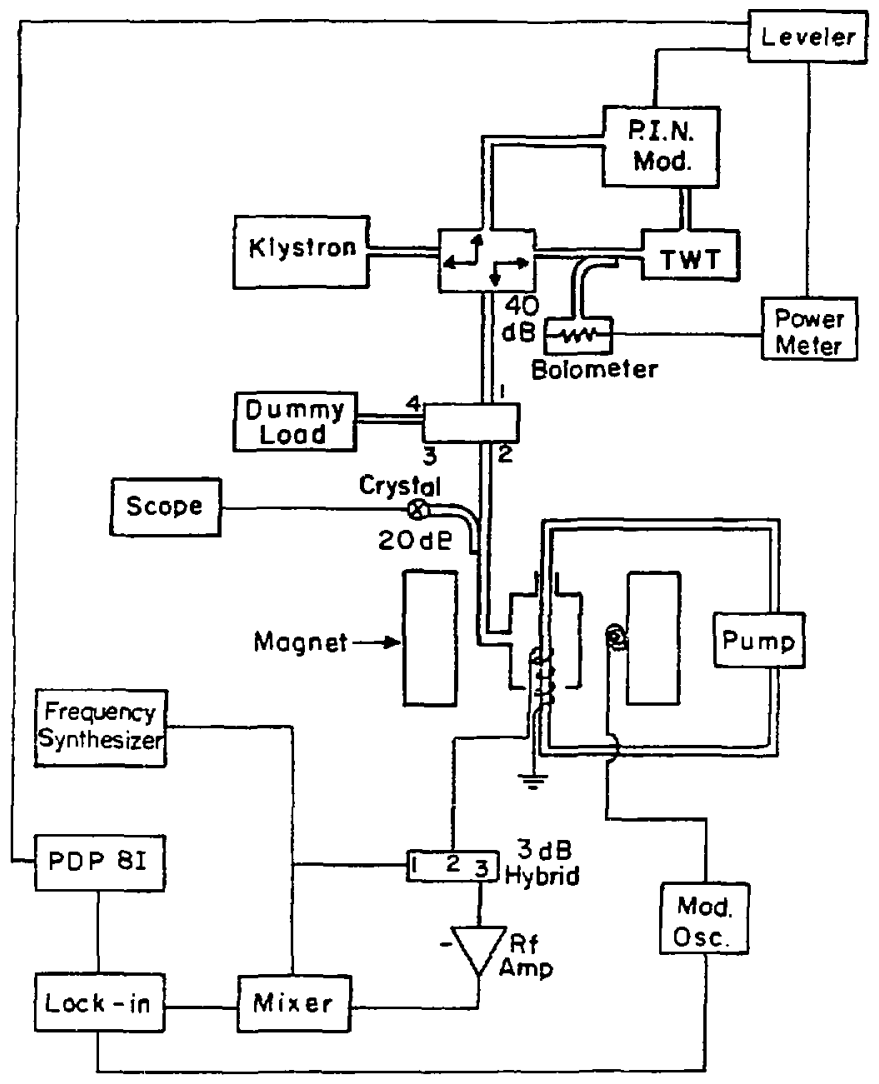

$X B L 827-5968$

Fig. 5. Block diagram of the flowing sample DNP spectrometer. 
rise of the solution upon passing through this cavity is less than $1.5^{\circ} \mathrm{C}$ for $15 \mathrm{~W}$ of incident power.

The microwave cavity is a Varian V -4535 large sample cylindrical cavity. The main virtue of this cavity appears to be its length along the cylindrical axis. Because the enhancement is expected to build up as in Eq. (4.7), the larger the cavity length $d$, is, the more enhancement there will be for a given flow rate and $\left(H_{1}^{2}\right)$. Enhancements in this cavity, with a $6.9 \mathrm{~cm}$ length and a $4.1 \mathrm{~cm}$ diameter were generally a factor of two larger than for a cavity $2.9 \mathrm{~cm}$ in length and $4.9 \mathrm{~cm}$ diameter. This was true even though the smaller cavity would be expected to have a larger value of $\left\langle H_{1}^{2}\right\}$. The larger cavity can be operated in either the $T E_{012}$ or the $T E_{011}$ mode. The resonant frequency of the $T E_{011}$ mode with the sample in place is $9.0 \mathrm{GHZ}$. The loaded $Q$ is approximately 2000 .

The initial source of $X-b$ and radiation is a Varian $V-58 C$ k Tystron. Power from the klystron can be switched to the cavity directly for tuning purposes or amplified by a Varian VA-618M travelling wave tube (TWT). The THT is capable of supplying $20 \mathrm{~W}$ of continuous power. Power from the tube is leveled by controlling the input power with an HP $8735 B$ P.I.N. modulator and the leveling circuit shown in fig. 6 . The klystron is not locked to the cavity. Reflected power is monitored with a cryst The kijstron reflector voltage can be adjusted by hand to keep the .ucrowaves at the cavity resonance frequency. Klystron drift is not a serious problem for measurements on broad EPR lines. 


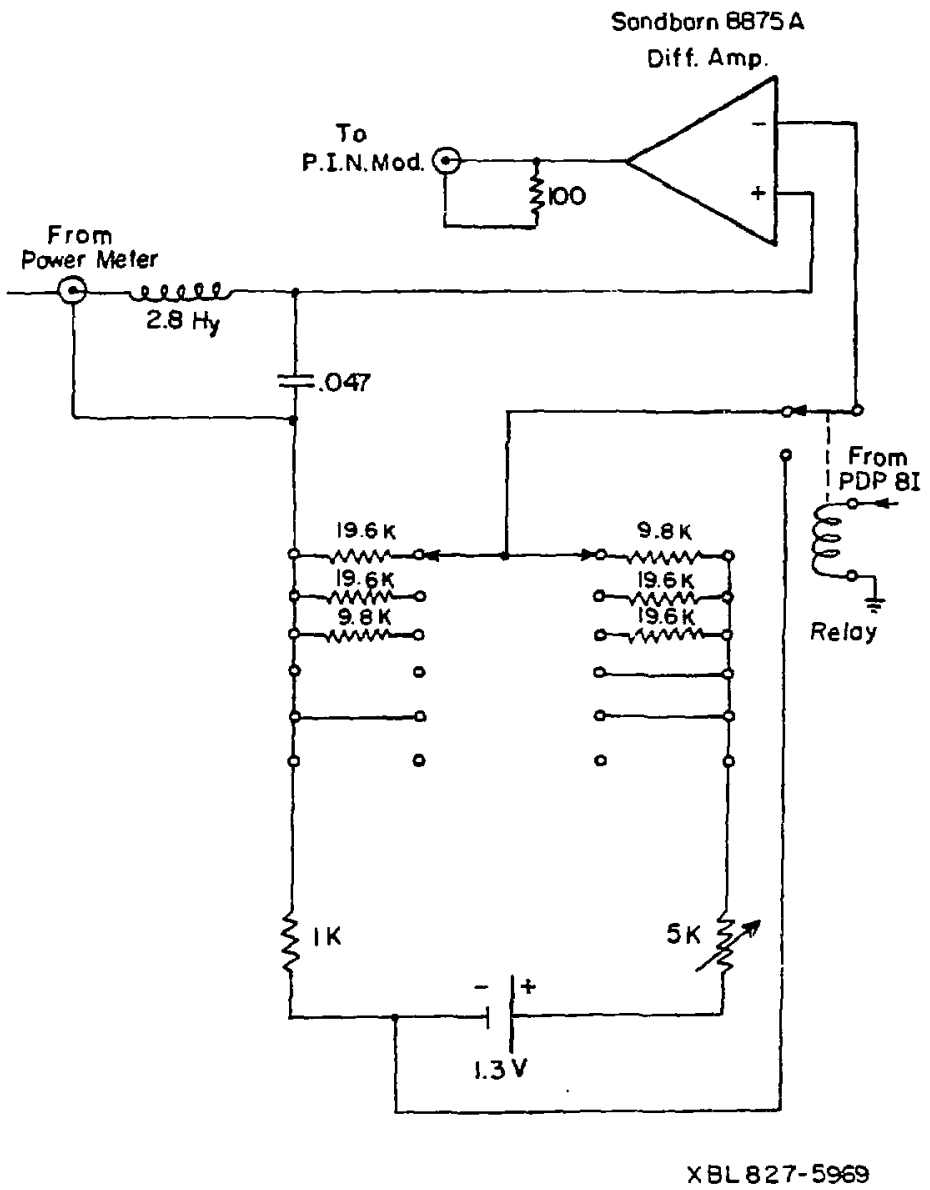

Fig. 6. Leveling circuit. 
The MR spectrometer is a continuous wave field modulated spectrometer. The MMR coil is located in the end cap of the cavity, as close to the interior of the cavity as possible in order to observe the induced polarization before it can decay away. Radio frequency radiation from a General Radio 1164-A frequency synthesizer is applied to the coil through a hybrid tee (Relcom Hybrid $\mathrm{H} 2$ ) and a network of tuning and matching capacitors. The capacitors are adjusted until no reflected power is observed from the third port of the tee when it is terminated with $50 \Omega$. The spectrometer is tunable to operate from $11.5-17 \mathrm{MHZ}$. The frequency of the synthesizer $c$ an be swept by the application of a voltage from a ramp generator (Datatron 2600-102). The AMR signal from the probe is taken from the third port of the tee and pre-amplified by two Watkins-Johnson thin film amplifiers. This setup supplies $30 \mathrm{db}$ of gain. The amplified signal is mixed with some of the original r.f. from the synthesizer in an HP10534 mixer. The magnetic field is modulated at $1 \mathrm{KHZ}$. One of the $1 \mathrm{KHZ}$ sidband signals from the mixer is amplified and phase detected with a Princeton Applied Research 124A lock-in ampl if ier.

Experiments are done under the contral of a PDP-8I computer, which is equipped with the AX08 lab peripheral. The microwaves can be turned on and off by the computer through a solid state relay and the leveling circuit. The ramp generator was modified to begin sweeps on computer command. The output signal from the lock-in amplifier is digitized and the intensities are calculated by the computer.

The magnetic field is supplied by a Pacific Electric Motors 12A-LI-EC, current stabilized electromagnet. The stability of the 
magnet is just barely adequate for these experiments. The amount of field drift makes averaging of individual MR specta impossible, but it is slow enough that intensities can be measured and averaged for several minutes without having to readjust the field. Homogeneity at the MR coil is adjusted by a Varian VK-3532 homogeneity controller.

A typical run would consist of taking several scans of the MMR spectrum, both with the micrawave power on and off. The computer averages the intensities and calculates the enhancements. The enhancement can be printed on a teletype and stored on magnetic tape for later manipulations. Typically, the enhancement as a function of magnetic field over the EPR line, the enhancement spectrum, is desiret1. For each point in the spectrum, before the enhancement can be measured, the Wir frequency must be set, tree probe tuned, and the MR signal found by adjustment of the magnetic field. This can le quite tedious. It takes trom one to several hours to obtain the enhancement spectrum with acceptable signal to noise. Figure 7 shows the enhancement spectrum of the $M_{I}=-1 / 2$ line of $\mathrm{VO}^{2+}$, which was taken in this manner. Each point is the result of averaging 50 AMR spectra. 


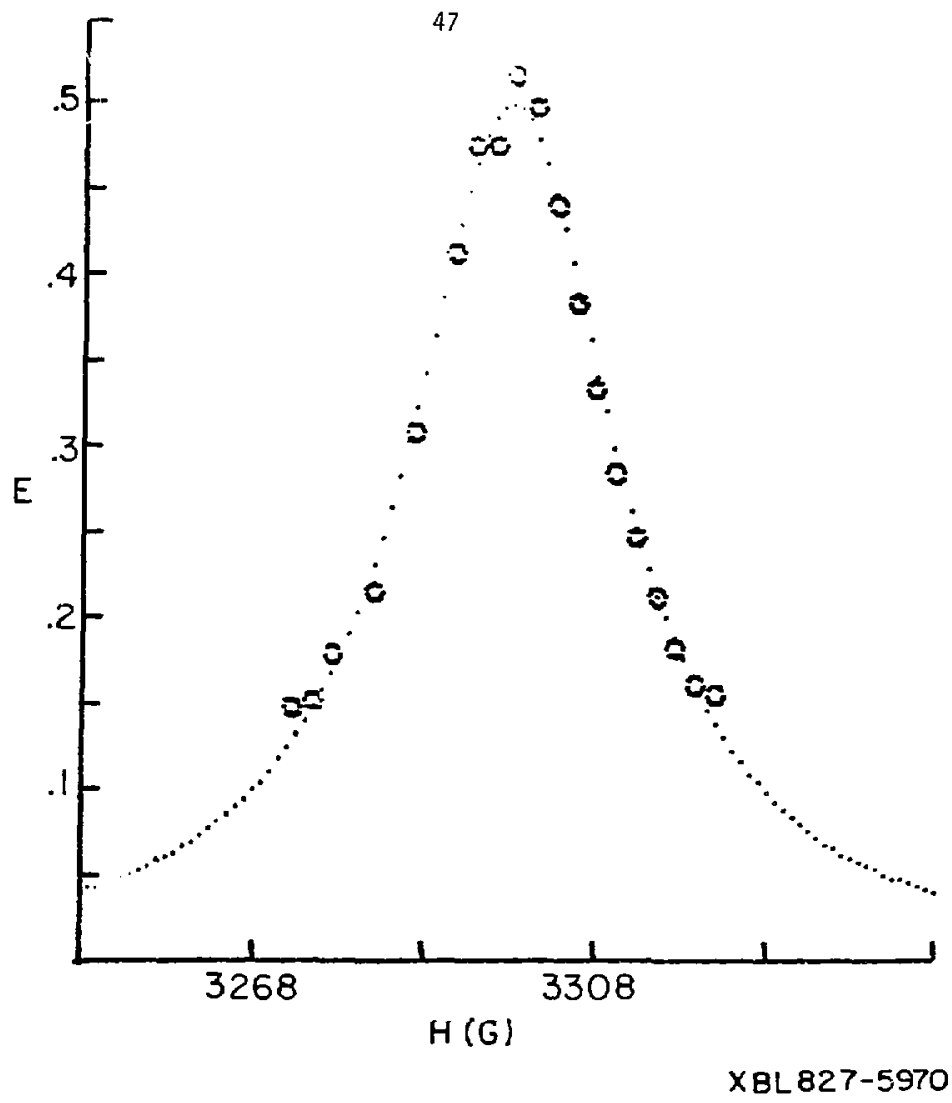

Fig. 7. ONP enhancement spectrum of the $M_{1}=-1 / 2$ line of $\mathrm{V}^{2+}$. 


\section{Flow Spectrometer Results}

\section{A. Vanady1 Ion}

1. Introduction

The ERR spectrum of $V^{2+}$ (vanady 1 ) ion in aqueous solution was first reported by Garif'ianov and Kozyrev (1954) and Pake and Sands (1955). Pake and Sands noted the appearance of eight lines due to hyperfine coupling to the Vanadium-51 nucleus $[I=7 / 2$; natura? abundance 99.8 per-cent) and also cormented that each of the eight lines had a different width. This variation of the widths of the hyperfine lines has been the subject of a significant amount of experimental and theoretical interest.

$\mathrm{Vo}^{2+}$ ion has a single unpaired electron in a $3 \mathrm{~d}$ orbital. Because of the strong influence of the $V-0$ bond there is expected to be a strong tetragonal distortion to the aquated ion, $\mathrm{VO}\left(\mathrm{H}_{2} \mathrm{O}\right)_{5}^{2+}$. This results in a non-degenerate ${ }^{2} B_{2}$ ground state and an EPR spectrum with $l$ ines $n$ arrow enough to be easily observed at room temperature.

The variation of the linewidths with $M_{I}$ suggests a relaxation mechanism involving tumbling modulation of the anisotropic spinHåmiltonian parameters first predicted by McConrall (1956) and then refined by Kivelson (1960). Wilson and Kivelson (1966a) refined the theory even further and applied it to linewidth measurements on the neutral acetylacetonate complex of $\mathrm{vo}^{2+}$ in toluene and chloroform. They were able to explain the linewidths satisfactorily with the tumbling theory and a small, but predictable contribution from a spin-rotation mechanism. 
Rogers and Pake (1960) measured the linewidths of the aqueous $\mathrm{V}^{2+}$ ion at $\mathrm{x}-\mathrm{b}$ and $(9.25 \mathrm{GHz})$ and $\mathrm{K}-\mathrm{b}$ and $(24.3 \mathrm{GHz})$. They were able to fit the observed $x$-band $l$ inewidths to a polynomial in $M_{I}$ and, using a version of the Kivelson (1960) theory, predict the K-band linewidths with moderate accuracy.

McCain and Myers (1967) observed aqueous $\mathrm{VO}^{2+}$ at S-band (3 GHz) and $X-b$ and. They also measured the anisotropic spin-Hamiltonian parameters $g_{\|}, g_{1}, A_{\|}$, and $A_{1}$ of $v 0^{2^{+}}$in a perchloric acid glass to make a more direct use of the theory. The anisotropic parameters of the aquated ion cannot be determined from measurements on the liquid solution. They chose to measure the anisotropic parameters in perchloric acid glass to take advantage of the non-complexing properties of the perchlorate ion. It was hoped that the environment of the $\mathrm{vo}^{2+}$ ion in the glass did not differ significantly from that of tie aquated ion in liquid solution. They used the measured anisotropic parameters in Eqs. (2.1)-(2.5). Assuming a tumbling correlation time of $3.5 \times 10^{-11} \mathrm{sec}$ at $30^{\circ} \mathrm{C}$, they found that the combination of the Kivelson theory and spin-rotation underestimated the $M_{I}$ independent component of the 1 inewidth by $3.1 \mathrm{G}$ out of $12.7 \mathrm{G}$ at $\mathrm{X}$-band and $5.9 \mathrm{G}$ out of $21.3 \mathrm{G}$ at $5-b$ and. They felt that perhaps the difference between the residuat widths could be due to terms in the ineory proportional to $(a / \omega)^{2}$ which were discarded by Kivelson. These tenms would be much more important at S-band. Since the lines at S-band are considerably wider than those at $x-b$ and, and since parti:l overlap of the lines would make accurate determination of the linewidths more difficuit, the $x-b$ and data should be considered more reliable. 
Angerman and Jordan (1971), and Chang (1971) measured the linewidths of aqueous $\mathrm{VO}^{2+}$ ion as a function of temperature at $x-b$ and. Angerman and Jordan found a residual width which decreased witr: increasing temperature. They used the experimentally detemined $\gamma$ parameter to estimate the correlation time. This correlation time was then used in Eqs. (2.1)-(2.5) to calculate the theoretical linewidth. Chang's results were qualitatively similar, and they showed a residual width which decreased with increasing temperature, although the residual width was smaller with a less pronounced temperature dependence. Chang determined his linewidths by fitting the observed spectra to a lineshape function composed of the sum of derivatives of Lorentzians. This method is expected to avoid errors due to partial overlap of the lines. Angerman and Jordan measured the peak to peak separations directly from their spectra. Perhaps this explains the difference between the two sets of data, especially at low temperatures where overlap is more severe.

At $20^{\circ} \mathrm{C}$, Chang found that $2.2 \mathrm{G}$ of the 1 inewidtr was unexplained by tumbling theory plus spin-rotation. Lewis and Morgan (1968) suggested that the residual width was due to unresolved proton superhyperf ine coupling. Chang measured spectra in $D_{2} O$ and found residual widths similar to the nomal aqueous system. If the residual width were due to superhyperf ine it should have been considerably reduced in $\mathrm{D}_{2} \mathrm{O}$. 
Reuben and Fiat (1969) detemined the $\mathrm{vo}^{2+}$-proton superhyperfine constant from the chemicai shift of the proton NMSi spectrum. They determined a superhyperfine constant of $1.1 \mathrm{G}$. Ianuzzi, Kubiak, and Rieger (1976) measured the linewidths of $\mathrm{Vo}\left(\mathrm{H}_{2} \mathrm{O}\right)_{5}^{2+}$ in $1 \mathrm{M} \mathrm{Na}\left(\mathrm{ClO}_{4}\right)_{2}$ solution as a function of temperature. They also did computer simulations to determine the effect of proton superhyperf ine on the observed linewidths. They found that a $1 \mathrm{G}$ superhyperf ine contribution from eight equivalent protons would only contribute $0.3 \mathrm{G}$ to the average linewidths. When they corrected for this superhyperf ine interaction this left them with a residual linewidth of $3.0 \mathrm{G}$. They also measured iinewidths of $\mathrm{VOCl}_{2}$ solutions in $\mathrm{D}_{2} \mathrm{O}$ and $\mathrm{H}_{2} \mathrm{O}$, finding a $0.4 \mathrm{G}$ larger resicual width for the protonated species, in close agreement to the ir expected excess of 0.36 .

Some of the residual widths observed seem to depend on the concentrations and types of anions used in the measurements. Yet, in the dilute cloride solutions used by Ianuzzi, Kubiak and Rieger, and the dilute perchlorate solutions exariined by Chang, there remains a residual width which $c$ annot be accounted for by the presence of excessive concentrations of anions or unresolved proton superhyperfine coupling.

MCCain and Myers (1967) used ONP to measure the relative $\mathrm{T}_{1 \mathrm{e}}$ of the eight $\mathrm{V}^{2+}$ hyperf ine lines and they compared them to the $\mathrm{T}_{1 \mathrm{e}}$ calculated from the nonsecular terms of the theory derived by Wilson and Kivelson (1966a). These terms yield

$$
\frac{1}{T_{1}}=\alpha_{1}+\beta_{1} M_{I}+r_{1} M_{1}^{2}+\sigma_{1} M_{I}^{3},
$$


with,

$$
\begin{aligned}
& \frac{a_{1}}{\tau_{R}}=\frac{1}{15}\left(\Delta Y B_{0}\right)^{2} u+\frac{1}{40} I(i+1) b^{2} u\left(1+f\left(\frac{a}{\omega_{0}}\right)\right) \\
& +\frac{3}{20} I(I+1) b^{2} u\left(1-f\left(\frac{a}{\omega_{0}}\right)\right)-\frac{1}{30} b \Delta Y B_{0} I(I+1)\left(\frac{a}{\omega_{0}}\right) u \\
& \frac{B_{1}}{\tau_{R}}=\frac{1}{5} b \Delta \gamma B_{0} u-\frac{2}{15}\left(\Delta \gamma B_{0}\right)^{2}\left(\frac{a}{\omega_{0}}\right) u(1+f) \\
& \quad-b^{2} I(I+1) u\left[\frac{7}{20}\left(\frac{a}{\omega_{0}}\right) f+\frac{3}{20}\left(\frac{a}{\omega_{0}}\right)\right] \\
& \quad+\frac{1}{20} b^{2}[2 I(I+1)-1]\left(\frac{a}{\omega_{0}}\right) u \\
& \frac{Y}{\tau_{R}}=-\frac{1}{40} b^{2} u-b \Delta Y B_{0} u\left[\frac{2}{15}\left(\frac{a}{\omega_{0}}\right)+\left(\frac{2}{5}\right) f\left(\frac{a}{\omega_{0}}\right)+\frac{3}{20} b^{2} u f\left(\frac{a}{\omega_{0}}\right)\right] \\
& \delta / \tau_{R}=\frac{1}{20} b^{2} u\left(\frac{a}{\omega_{0}}\right)(1+f)
\end{aligned}
$$

where the meaning of the variables in the expressions are given following Eq. (2.5). These expressions assume axial symmetry of the paramagnetic complex. In the ir analysis, MeCain and Myers assumed all of the residual linewidth was a $T_{1}$ process. When they assumed that $\alpha_{1}$ (measured) $=\alpha$ (theoretical), the measured values of $\gamma_{1}$ and $\delta_{1}$ agreed we?l with the theory. However, there was a large discrepancy in $B_{1}$. There are of course no grounds for expecting $x_{1}$ (measured) to be the 
same as the theoretical $\alpha_{1}$. A discrepancy in the a's would most likely show up as a discrepancy in the a's when this comparison method is used. As in the case of the linewidth measurements the difference between theory and experiment was ascribed to the neglect of terms in $\left(a / \omega_{0}\right)^{2}$ in the theory.

There are many reasons to measure the DNP of $\mathrm{V0}^{2+}$ ion at $\mathrm{X}$-band. The use of DNP to measure absolute $T_{1 e}$ requires a standard with a known $\mathrm{T}_{10}$. Because the linewidths of the $10^{2+} E P R$ spectrum are well explained by theory (with the exception of a small residual width), it may be possible to compare other ions with $\mathrm{vo}^{2+}$. The theoretical value of $T_{1 e}$ could serve as the absolute guidepost. The terms in $\left(a / \omega_{0}\right)^{2}$ which were neglected in the theory are less important at $x$-b and than at 5-band. Measurements of the relative $T_{1 e}$ values of the eight $v^{2+}$ hyperf ine 1 ines would serve to reinforce confidence in the theory. Since at $x$-bani $\omega_{e}{ }^{\top} c \geq 1$, the non-secular contributions to the 1 inewidth are smaller than the secular contributions. If the residual width were a $T_{1}$ process it should contribute a larger fraction to $T_{1 e}$. It would have a large effect on the relative $T_{l e}$ 's and should be easy to detect. A superhyperf ine contribution would not affect $T_{1 e}$, so relative $T_{l e}$ measurements could shed some light on the residual width question for $\mathrm{VO}\left(\mathrm{H}_{2} \mathrm{O}\right)_{5}^{2^{+}}$.

\section{Experimental Methods}

VO $\left(\mathrm{ClO}_{4}\right)_{2}$ solutions were prepared from $\mathrm{VOSO}_{4}$ (Eastman) by the ion exchange method described by Chang (1971). Solutions were anaiyzed 
for $\mathrm{V}^{2+}$ by monitoring the absorbance at $765 \mathrm{~nm}$. The molar extinction coefficient $\varepsilon$, was taken as 16.98 (Santini, Hazel, and McNabb, 1952).

DNP measurements were made with the apparatus described in Chapter IV. The microwave power level was $10 \mathrm{~W}$. The flow rate was $150 \mathrm{cc} / \mathrm{min}$. The temperature was not controlled, but it did not deviate from the room temperature, $23^{\circ} \mathrm{C}$, by more than $1^{\circ} \mathrm{C}$ during the course of the experiment.

3. Results and Discussion

Figure a shuits the DNP spectra of the $M_{I}=-7 / 21$ ine and $M_{I}=$ $7 / 2$ line of $\mathrm{VO}^{2+}$. Spectra of this sort were taken for all eight hyperfine lines. This was done primarily to accurately determine the 1 inecenters, because a second-order perturbation treatment does not predict the linecenters of the outer lines correctly. The enhancement data for each line were fitted to a function of the form

$$
E=\frac{E_{0} H_{0}}{H\left(I+\left(H-H_{0}\right)^{2} / \delta^{2}\right)} \text {, }
$$

where $E_{0}$ is the enhancement at 1 ine centers, $H_{0}$ is the linecenter and $\delta$ is the half-width at half height. The fitted half-widths were always slightly larger than the equivalent widths measured from the EPR spectrum. This was most likely due to overlap from adjacent lines. Once the linecenters were determined more data were taken for each 1 ine at the linecanter field. The uncertainty in each of the measured enhancements was less than \pm .005 . 


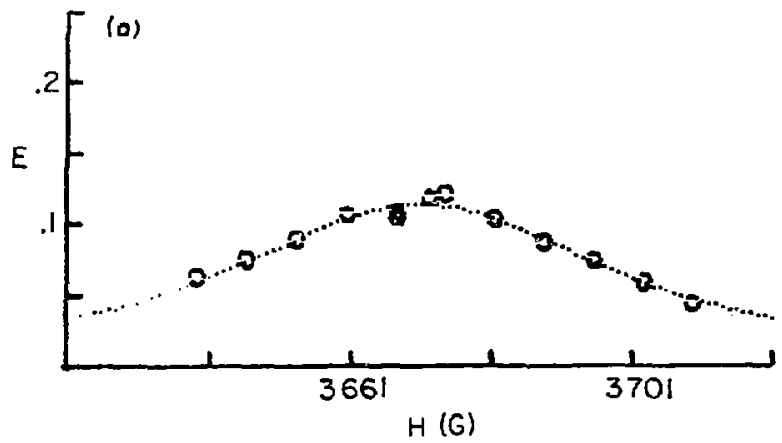

Fig. 8a. The DNP enhancement spectrum of the $M_{I}=7 / 2$
line of $V 0^{2+}$

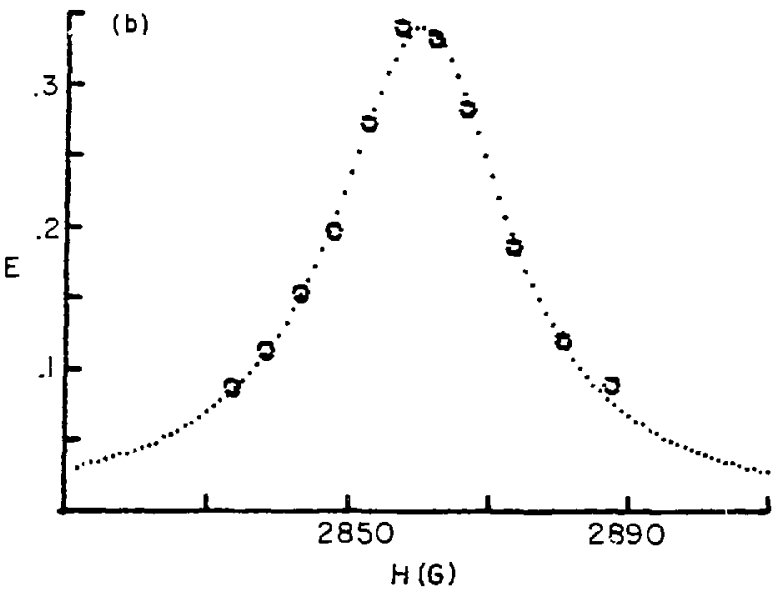

XBL $827-597$ I

Fig. 8b. The enhancement spectrum of the $M_{I}=-7 / 21$ ine of $\mathrm{V}^{2+}$. Both spectra in this igure were taken with $10 \mathrm{~W}$ of microwave power. 
The measured enhancements were corrected for overlap by solving eight equations in eight unknowns of the form

$$
E_{M_{I}} \text { (measured) }=\sum_{M_{I}=-\frac{7}{2}}^{\frac{7}{2}} \frac{E_{M_{I} \text { (actual) }}\left(1+\frac{\left(H-H_{\left.M_{l}\right)^{2}}^{2}\right.}{\delta_{M_{I}}^{2}}\right)}{{ }^{2}}
$$

where ${ }^{s} M$ and $H_{M}^{0}$ are the half-width and linecenter of the $M_{I}$ 'th hyperf ine $l$ ine, and $H$ is the field of the measured enhancement, $E_{M}$ (measured). The uniziowns are the $E_{M}$ (actual)'s. The overlap correction: are small and can be calculated nearly as accurately by only considering the effect of lines adjacent to the line of interest.

Numbers proportional to $\mathrm{T}_{1 \mathrm{e}}$ can be obtained by multiplying the overlap corrected enhancement by the linewidth and linecenter of the line. The numbers are then nomalized with respect to the line having the 1 argest $\mathrm{T}_{10}$. These ratios are given in Table $I$. The linewidths used in Table I are interpolated from EPR measurements by Chang (1971). The linewidths from EPR measurements are presumed to be more accurate than those abtained from the DNP fits.

The calculated values of $T_{1 e}$ shown in Table 1 are detenined from Eqs. (5.1)-(5.4) and (2.8) using spin-Hamiltonian parameters determined by chang. The correlation time used in these calculations was $4.6 \times 10^{-11} \mathrm{sec}$. It was calculated using Eq. (2.6) and the hydrodynamic radius determined by Chang. The residual width was 
Table: I. DNP Parameters for $\mathrm{VO}^{2+}$

\begin{tabular}{ccccc}
\hline$M_{I}$ & $H_{0}(G)$ & $4 H(G)$ & $T_{1}$ (Measured) & $T_{1}$ (Calculated) \\
\hline$-7 / 2$ & 2860.4 & 16.48 & 0.78 & 1.00 \\
$-5 / 2$ & 2963.7 & 12.98 & 0.92 & 0.93 \\
$-3 / 2$ & 3071.3 & 11.55 & 0.87 & 0.86 \\
$-1 / 2$ & 3182.5 & 11.92 & 0.84 & 0.80 \\
$1 / 2$ & 3299.1 & 13.83 & 0.81 & 0.75 \\
$3 / 2$ & 3419.2 & 17.36 & 0.80 & 0.71 \\
$5 / 2$ & 3583.6 & 22.31 & 0.79 & 0.68 \\
$7 / 2$ & 3671.3 & 28.99 & 0.78 & 0.66 \\
\hline
\end{tabular}


included as a contribution to $T_{1 e}$ in the calculation made here. The residual width measured by Chang at $23^{\circ} \mathrm{C}$ would contribute $2.7 \times 10^{7} \mathrm{sec}^{-1}$ to $1 / T_{1 \mathrm{e}}$ if $i$ ts contribution to $T_{1 \mathrm{e}}$ and $T_{2 \mathrm{e}}$ were equal. The calculated contribution to $1 / \mathrm{T}_{1 \mathrm{e}}$ from spin-rotation is $1.5 \times 10^{7} \mathrm{sec}^{-1}$. The calculated contribution from tumbling to $1 / \mathrm{T}_{\mathrm{le}}$ for the $M_{I}=-7 / 21$ ine is $1.9 \times 10^{7} \mathrm{sec}^{-1}$. If the residual width does contribute equally to $T_{1 e}$ and $T_{2 e}$, then $i$ ts contribution to the total $\mathrm{T}_{1 \mathrm{e}}$ would be quite significant.

Comparison of the measured ani calculated $T_{1 e}$ ratios shows reasonable qualitative agreement, since $T_{1 e}$ is seen to decrease monotonically with increasing $M_{I}$ for both the measured and calculated values. However, one can see that the spread of the measured $T_{1 e}$ ratios is less than that given by the theory. Since the residua) width contributed equally to the calculated $T_{1 e}$ value for each $M_{I}$, diminishing its contribution would anly make the quantitative agreement between the measured and calculated ratios worse. This disagreement is larger than expected from the experimental uncertainty and seems to be systematic, since the measured $T_{1 e}$ ratios appear consistently low. This is similar to the results reported by McCain and Myers (1967). They also had a smaller spread of the $T_{l e}$ ratios for the experimentally determined numbers. This may point to a flaw in the tumbling theory, but there are also complications in the interpretation of the experimental results.

The experimental interpretation is complicated by cross relaxation among the $v^{2+}$ EPR nyperf ine lines. The tumbling modulation of $\mathrm{g}$ 
and $A$ anisotropy leads to terms in the relaxation proportional to $S_{ \pm} I_{z}, S_{ \pm} I_{ \pm}$and $S_{ \pm} I_{\mp}$, where I refers to the spin of the Vanadium-51 nucleus. These terms cause a leakage of polarization out of an irradiated line. There is in fact not a single $T_{1}$ for each line, but there are several. A steady state saturation experiment carinot measure these relaxation times separately. What is actually measured is a quantity called the saturation parameter, which is a function of all the processes which resist saturation of an irradiated line. The DNP experiment is somewhat different. It measures $\left\langle S_{z}\right\rangle-S_{0}$. Because of cross relaxation, the irradiation of one set of spins as represented by a hyperf ine line can cause a polarization change in a non-irradiated line, making $\left\langle S_{z}\right\rangle-S_{0}$ non-zero for the nonirradiated spins. The DNP enhancement is proportional to all of the electron spin polarization. The assignment of relaxation times, or more precisely, saturation parameters, to individual lines on the basis of ONP results requires a more careful consideration of the cross relaxation process.

Freed (1965, 1972) considered saturation and double resonance experiments in the presence of cross relaxation. Figure $9 a$ shows a schematic level diagram for an electron coupled to a single nucleis of spin 1/2. The possible electron spin relaxation transitions are indicated by the arrows. Note that $W_{N}$ on $j y$ involves a nuclear sp in flip, $W_{e}$ only an electron spin $f l i p$, and $W_{x}$, and $W_{x}$ involve flips of both. If the 2 to 1 transition is saturated, and the absorption of the 3-4 transition is measured using low power, Freed (1972) finds that the percentage reduction of the absorption signal, $R$ is given by 
60

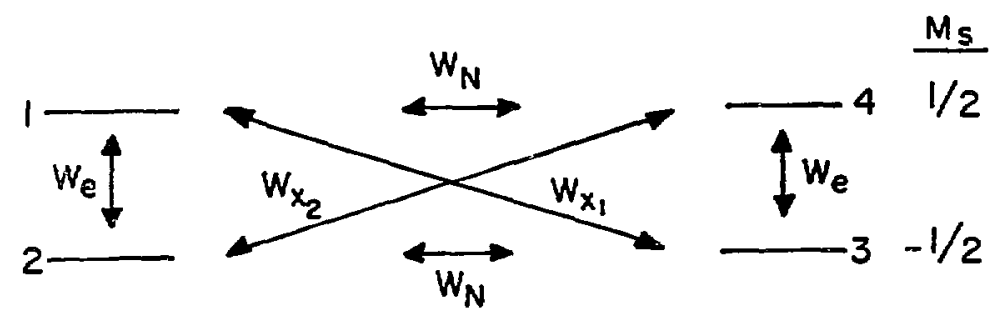

(a)

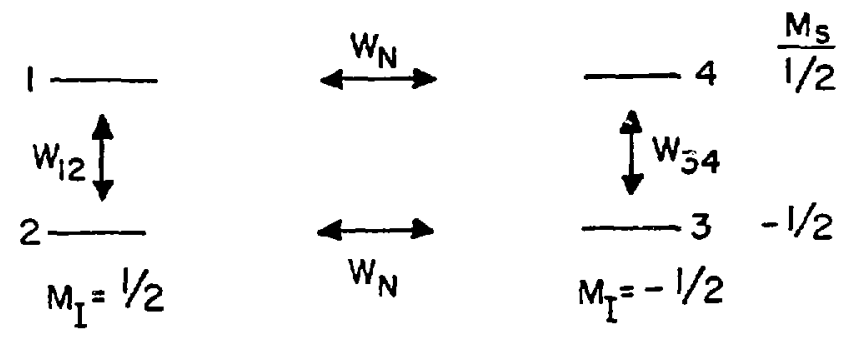

(b)

XBL 827-5972

Fig. 9. 


$$
R=\frac{100\left(w_{N}^{2}-w_{x_{1}} w_{x_{2}}\right)}{w_{e}\left(2 W_{N}+w_{x_{1}}+w_{x_{2}}\right)+\left(w_{N}+w_{x_{1}}\right)\left(w_{N}+w_{x_{2}}\right)}
$$

If $W_{N} \gg W_{x_{1}}, w_{x_{2}}$, then saturating the 2 to 1 transition also saturates the 3-4 transition, resulting in a decrease in signal intensity. If $W_{x_{1}}, W_{x_{2}} \gg W_{N}$ saturating the 1-2 transition results in an increase of signal in the 3-4 transition.

The rates $w_{N}, w_{x_{1}}$ and $w_{x_{2}}$ can be calculated for $\mathrm{Vo}^{2+}$ from the theory (Wilson and Kivelson, 1966a, P. 164). At $X$-band we have $\omega_{e}^{2} \tau_{R}^{2}=7$. The rate, $w_{N}$, which only requires a nuclear spin flip, is about eight times larger than $W_{x_{1}}$ and $W_{x_{2}}$, which are proportional to $1 /\left(1+\omega_{e}^{2} \tau^{2}\right)$. Thus saturation of one $v 0^{2+}$ line will lead to satura-

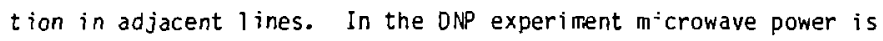
limited and only partial saturation is possible. It is necessary to consider the effect of partial saturation on total $\left\langle S_{2}\right\rangle-S_{0}$.

Consider the four level system in Fig. 9b, which now neglects $w_{x_{1}}$ and $w_{x_{2}}$, but has a different $w_{e}$ for each hyperfine transition. It can be shown (Freed, 1972) that

$$
\left(\left\langle S_{z}\right\rangle-S_{0}\right)_{\text {tota }}=\frac{-S_{0}\left(\Omega_{p}+\Omega_{o p}\right) T_{2 p} d^{2}}{2\left(1+d^{2} T_{2 p} \Omega_{p}\right)},
$$

when one of the transitions is partially saturated at resonance by an oscillating field of magnitude $H_{1}$. Here $d=(1 / 2)_{r_{e}} H_{1}, \Omega_{p}$ is the saturation parameter of the pumped resonance, $\Omega_{o p}$, is a cross-saturation 
parameter and $T_{2 p}$ is the spin-spin relaxation time. In the case of Fig. 9b,

$$
\Omega_{0}=\frac{2\left(w_{N}+2 w_{34}\right)}{2 W_{12} W_{34}+w_{N}\left(w_{12}+w_{34}\right)} \text {, }
$$

and

$$
\Omega_{o p}=\frac{2 W_{N}}{2\left(W_{12} W_{34}\right)+W_{N}\left(W_{12}+W_{34}\right)} .
$$

Because of the limited power in our DNP experiment $d^{2} T_{2 p} p_{p}<1$ so

$$
\left.\left(\left\langle S_{z}\right\rangle-S_{0}\right)_{\text {tota }}\right)=\frac{-S_{0}\left(\Omega_{p}+s_{\partial p}\right) T_{2 p} d^{2}}{2} .
$$

If $W_{N}=0$ (no cross relaxation), then

$$
\left\langle S_{2}\right\rangle-S_{0}=\frac{-S_{0}}{W_{12}} T_{2 p} d^{2}
$$

This reduces to the familiar result

$$
\left\langle\left\langle S_{z}\right\rangle-S_{0}\right\rangle_{\text {total }}=\frac{-S_{0} \gamma^{2} H_{1}^{2} T_{1 p}{ }^{\top} 2 p}{2},
$$

if we made the identification $T_{1 p}=1 / 2 \mathrm{~W}_{12}$. If we now allow cross reiaxation, $w_{N} \neq 0$, but make $w_{12}=w_{34}$, then 


$$
\left(\left\langle\dot{S}_{z}\right\rangle-S_{0}\right)_{\text {tota } 1}=\frac{-S_{0} 2\left(2 W_{N}+2 W_{12}\right) T_{2 p} d^{2}}{4 W_{12}\left(W_{12}+W_{N}\right)}=\frac{-S_{0} T_{2 p} d^{2}}{W_{12}}
$$

This is the same result as if there were no cross relaxation. When the saturating power is limited, the opening of a new pathway for saturatior, by cross relaxation does not necessarily result in more saturation being observed. In this case, where $w_{12}=W_{34}$, the same total saturation is observed, only it is shared between the two spin systems. As $W_{N}$ increases, the amount of saturation in the spin systerm which is not being pumped increases, but the total amount of saturation in the two spin systems remains the same. A final interesting limiting case is that of $W_{N} \gg W_{34}, W_{12}$. Here we have

$$
\left(\left\langle S_{z}\right\rangle-S_{0}\right)_{\text {total }}=\frac{-S_{0} 2 T_{2 p} d^{2}}{\left(w_{12}+w_{34}\right)}
$$

In this limit cross rejaxation leads to the measurement of an apparent spin lattice relaxation rate which is an average of the rates of the two transitions involved.

The $\mathrm{V}^{2^{+}}$case with eight 1 ines is of course more complicated than the four leve? system, but the effect of cross relaxation on the experimental results can be understood qualitatively on the basis of this model. Cross relaxation makes the nidasured enhancements reflect a weighted average of the spin lattice relaxation rates of all the lines. The weighting of the average depends on the size of $w_{N}$ compared to the spin-lattice rates. Clearly if $W_{N}$ were totally 
dominant, the same apparent spin-lattice relaxation rate would be measured no matter which line was saturated. Our measurements do not support this result. Using the pseudo-secular terms of the tumbling theory (Wilson and Kivelson 1966, p. 164), $W_{N}$ can be calculated. For the $M_{1}=1 / 2$ line $W_{N}=1 \times 10^{8}$. If the residual width and spiriratation effects are included, $W_{e}$ for this line is $8 \times 10^{7}$. In this case cross relaxation is significant, but not overly dominant. Only averaging of relaxation rates with adjacent lines would oe significant. $W_{N}$ becomes even less significant far the outer lines of the $\mathrm{vo}^{2+}$ spectrum. The net result is that the spread of the observed $T_{1 e}$ 's should be smaller than is predicted by theory. This is qualitatively wrat is observed.

Cross relaxation should not hinder the use of $\mathrm{VO}^{2+}$ as a DMP standard too severely, since the spin-lattice relaxation rates of adjacent lines are very similar. The results of these relative reldxation measurements $y$ : enouth conf jdence in the theory ti make our use of $\mathrm{VO}^{2+}$ as a DNP standard quite reasonable.

\section{Copper Ethylenediamine}

1. Introduction

Another system for which the EPR 1 inewidths are reasonably well understood is the ethylenediamine complex of copper, $\mathrm{Cu}\left(\mathrm{en}, 2\left(\mathrm{H}_{2} \mathrm{O}\right)_{2}^{2^{+}}\right.$. DNP measurements on this ion should serve as a good test of the experimental technique. In particular, these measurements should test the usefulness of $\mathrm{V}^{2+}$ as a $T_{1 e}$ standard. 
$\mathrm{Cu}(\mathrm{en})_{2}\left(\mathrm{H}_{2} \mathrm{O}\right)_{2}^{2^{+}}$is a $\mathrm{d}^{9}, \mathrm{~S}=1 / 2$ ion. The abundant isotopes of copper, ${ }^{63} \mathrm{Cu}$ (69 percent natural abundance), and ${ }^{65} \mathrm{Cu}$ ( 31 percent natural abundance) both nave nuclear sp in $I=3 / 2$. The gyromagnetic ratios of the two isotopes are sufficiently similar that the difference in the hyperfine coupling constants between ${ }^{63} \mathrm{Cu}$ and ${ }^{65} \mathrm{Cu}$ can be neglected for the interpretation of our $\mathrm{Cu}(\mathrm{en})_{2}\left(\mathrm{H}_{2} \mathrm{O}\right)_{2}^{2+}$ spectra. Lewis, Alei, and Morgan (1966b) measured the EPR spectra of $\mathrm{Cu}(\mathrm{en})_{2}\left(\mathrm{H}_{2} \mathrm{O}\right)_{2}^{2+}$ from $-10^{\circ} \mathrm{C}$ to $100^{\circ} \mathrm{C}$. The ${ }^{63} \mathrm{Cu}$ hyperf ine coupling constant is $-87.5 \mathrm{G}$. The spectrum appears as a partially resolved quartet over this temperature range. The individual hyperf ine lines have different widths, suggesting a contribution to the linewidth from the tumbing mechanism proposed by McConnell (1956). The g values differ significantly from the free electron value, so spin-rotation should also contribute substantially to the relaxation. There is also a nitrogen-14 superhyperfine coupling of $11.6 \mathrm{G}$ which cannot be neglected in the interpretation of the spectrum.

Because of the partially resolved nature of the ${ }^{63} \mathrm{Cu}$ hyperfine ines and the existance of the ${ }^{14} \mathrm{~N}$ coupling it is not possible to measure the linewidths directly from the spectrum. Lewis, Alei and Morgan extracted the linewidths by comparing the experimental spectra with computer simulations. They alsc measured the anisotropic spinHamiltanian parameters for the complex in frozen glass. They found that the temperature dependence of the difference between the width of the $M_{I}=-3 / 2$ line and the $M_{I}=3 / 2$ line could be adequately explained by the tumbling theory if a correlation time of $1.7 \times 10^{-11} \mathrm{sec}$ at 
$25^{\circ} \mathrm{C}$ was assumed. After the contribution to the linewidth from tumbling was subtracted, they found that the remaining width could be accounted for by spin-rotation. The application of Eq. (2.8) using their measured valuas of $g_{\|}=2.209$ and $g_{\perp}=2.047$ yielded a value for $\tau_{R}$ of $1.4 \times 10^{-11} \mathrm{sec}$. The temperature dependence of this remaining width was found to be somewhat less than the $T / 7$ dependence predicted by Eqs. (2.6) and (2.8). These correlation times estimated from tumbling and spin rotation were in fair agreement with the correlation time determined from the frequency dependence of proton MR reiaxation rates, $2 \times 10^{-11} \mathrm{sec}$ (Morgan, Murphy, and Cox, 1959).

\section{Experimenta] Methods}

Solutions of $\mathrm{Cu}(\mathrm{en})_{2}\left(\mathrm{H}_{2} \mathrm{O}\right)_{2}^{2+}$ were prepared by dissolving weighed amounts of $\mathrm{Cu}(e n)_{3} \mathrm{SO}_{4}$ (Alfa-Ventron) in distilled water. Both the tirst and second association constants for $\mathrm{Cu}^{2+}$ with ethylenediamine are large $\left(\log k_{1}=10.72, \log k_{2}=9.31\right)$, while the third is small ( $\log$ $k_{3}=-0.90$ ) (Bjerrum and Nielson, 1948), so only the $\mathrm{Cu}(e n)_{2}\left(\mathrm{H}_{2} \mathrm{O}\right)_{2}^{2+}$ species is expected to be present in solution.

DNP of $\mathrm{Cu}(\mathrm{en}){ }_{2}\left(\mathrm{H}_{2} \mathrm{O}\right)_{2}^{2+}$ was observed in the flawing sample spectrometer described previously. Enhancements were compared with measurements on separate solutions of $\mathrm{vo}\left(\mathrm{C}_{4}\right)_{2}$ at the same temperature and flow rate. Concentrations were adjusted to give approximately the same nuclear spin-lattice relaxation times according to measurements in the literature (Morgan, Murphy and Cox, 1959; Hausser and Laukien (1959)). 


\section{Results and Discussion}

DNP enhancement spectra were detemined for $\mathrm{Cu}(\mathrm{en})_{2}\left(\mathrm{H}_{2} \mathrm{O}\right)_{2}^{2+}$ at 3 , 23, 41 and $59^{\circ} \mathrm{C}$. Figures 10 and 11 show these spectra. In order to make use of the data, linecer:sr enhancements must be extracted. This was done by fitting each spectrum to $;$ f'dnction of the form

$$
E(H)=\sum_{I=-3 / 2}^{3 / 2} \sum_{j=-4}^{4} \frac{g(j) T_{I}}{\left(1+\frac{\left(H-H_{0 I}-j A\right)^{2}}{\delta_{I}^{2}}\right)}
$$

The summation is over the four ${ }^{63} \mathrm{Cu}$ hyperf ine lines which are each in turn split into nine superhyperfine lines by the scalar interaction with four equivalent ${ }^{14} \mathrm{~N}$ nuclei. $H$ is the magnetic field, and $\delta_{I}$ is the halfwidth at half height of the Ith ${ }^{63} \mathrm{Cu}$ line. $\mathrm{H}_{\mathrm{OI}}$ is the linecenter of the Ith hyperf ine line and $A$ is the ${ }^{14} \mathrm{~N}$ superhyperfine coupling constant. All of these parameters are available or calculable from information given by Lewis, Alei and Morgan (1966b). The factor $g(j)$ reflects the intensity of the $j$ th superhyperf ine $l$ ine. The relative intensities for $j=-4$ to 4 are $1 / 81,4 / 81,10 / 81,16 / 81$, $19 / 81,16 / 8 i, 10 / 81,4 / 81,1 / 81 . T_{1}$ is the number proportional to $T_{1 e}$ of the Ith hyperfine line. $T_{I}$ is detemined by linear least squares. The fitted functions are the dotted lines in the figures. The fitted values of $T_{I}$ are turned into enhancements by dividing by $H_{0} \delta_{I}$. Enhancements ranged from 0.19 to 0.06 . Uncertainties in these values estimated from the standard deviation of the $f i t$ and the inverse normal equation matrix (Shoemaker, Garland and Steinfeld, 1974) 
68

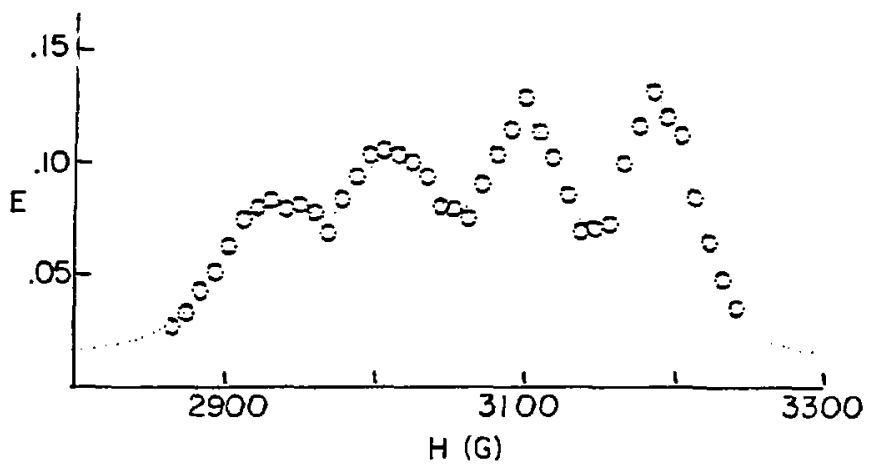

Fig. 10a. DNP spectrum of $\mathrm{Cu}(\mathrm{en})_{2}\left(\mathrm{H}_{2} \mathrm{O}\right)_{2}^{2+}$ at $3^{\circ} \mathrm{C}$.

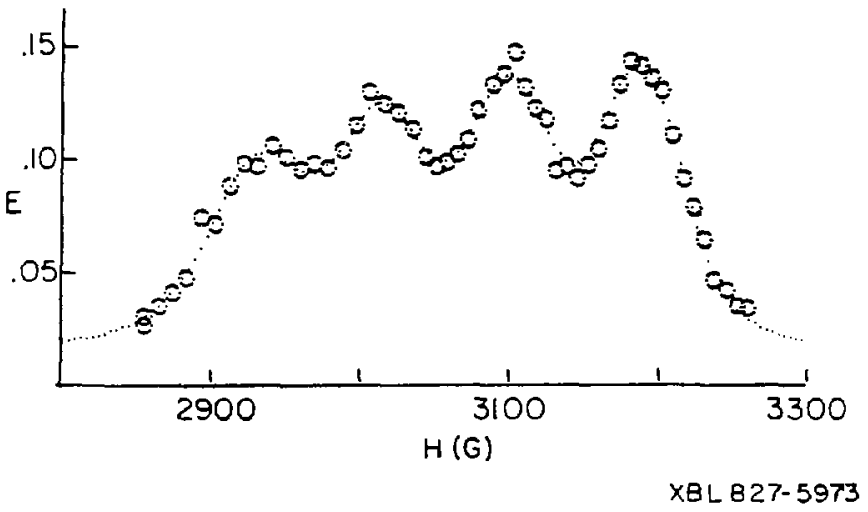

Fig. 10b. DNP spectrum of $\mathrm{Cu}(\mathrm{en})_{2}\left(\mathrm{H}_{2} \mathrm{O}\right)_{2}^{2+}$ at $23^{\circ} \mathrm{C}$. 


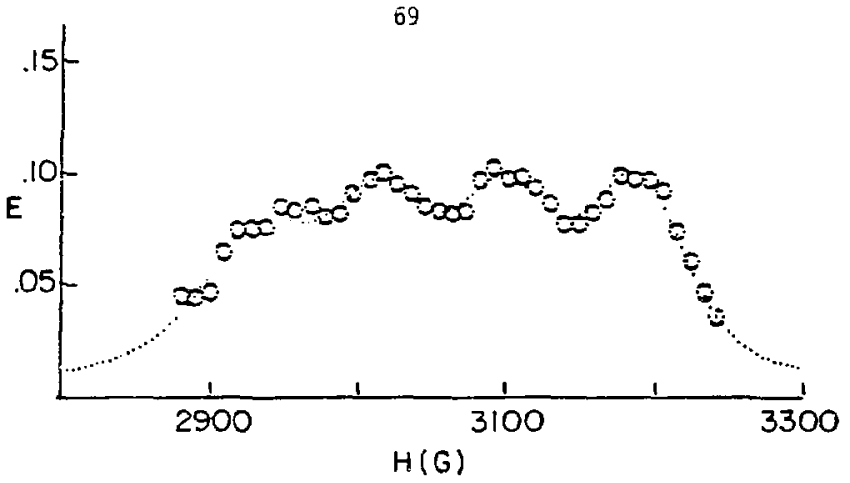

Fig. 1la. DNP spectrum of $\mathrm{Cu}(e \mathrm{en})_{2}\left(\mathrm{H}_{2} \mathrm{O}\right)_{2}^{2+}$ at $23^{\circ} \mathrm{C}$.

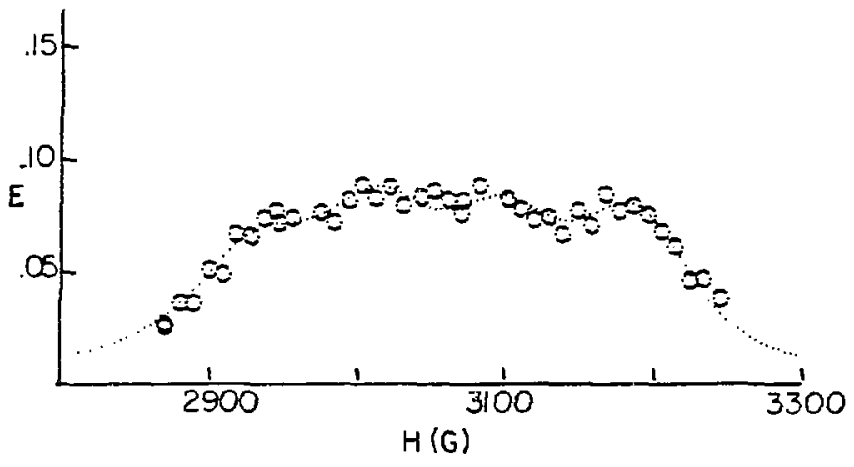

XBL827-5974

Fig. 11b. DNP spectrum of Cu(en $)_{2}\left(\mathrm{H}_{2} \mathrm{O}\right)_{2}^{2+}$ at $59^{\circ} \mathrm{C}$. 
ranged from 0.007 for the largest, enhancements to 0.004 for the smallest. Absolute $T_{1 e}$ 's were calculated from the relation

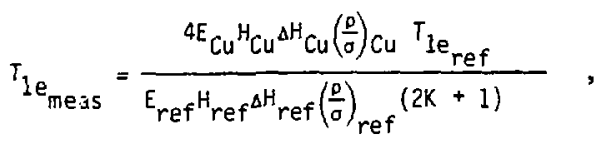

where $\mathrm{E}_{\mathrm{Cu}}, \mathrm{H}_{\mathrm{Cu}}$ and ${ }^{\Delta \mathrm{H}_{\mathrm{Cu}}}$ are the 1 inecenter enhancement, linecenter, and linewidth of the $63 \mathrm{Cu}$ hyperf ine line. $\mathrm{K}$ is the nuclear spin of the reference species. $E_{\text {ref }}, H_{\text {ref }}$ and $\Delta H_{\text {ref }}$ are the enhancement, linecenter and linewidth of the reference species. When $\mathrm{vo}^{2+}$ is used as a reference $T_{1}$ ref is calculated from theory using Eqs. (5.1)-(5.5) and (2.8), and spin-Haniltonian parameters determined by Chang (1971). The parameters 0 and $p$ were calculated for each species using Eqs. (3.13) and (3.14). The room temperature correlation time for $\mathrm{vo}^{2+}$, which was determined by Chang (1971) from EPR linewidth measurements, $4.6 \times 10^{-11} \mathrm{sec}$, was used in Eqs. (3.13) and (3.14). The value for $\mathrm{Cu}(e n)_{2}\left(\mathrm{H}_{2} \mathrm{O}\right)_{2}^{2+}$ determined from NMR relaxation measurements, $2 \times$ $10^{-1 I} \mathrm{sec}$, was used. The correlation times were assumed to vary with viscosity and temperature according to Eq. $(2,6)$.

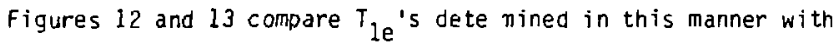
the $T_{2 e}$ measured by Lewis, Alei and Morgan (1968). At room temperature and above $T_{1 e}$ is very nearly equal :o $T_{2 e}$, with essentially the same temperature dependence. This is reasonable since spinrotation is expected to dominate the relaxation at these temperatures. Also $\tau_{R}$ for the tumbling interaction is short enough that the tumbling contribution to $T_{l e}$ is close to its limiting value of $7 / 6 T_{2}$. 


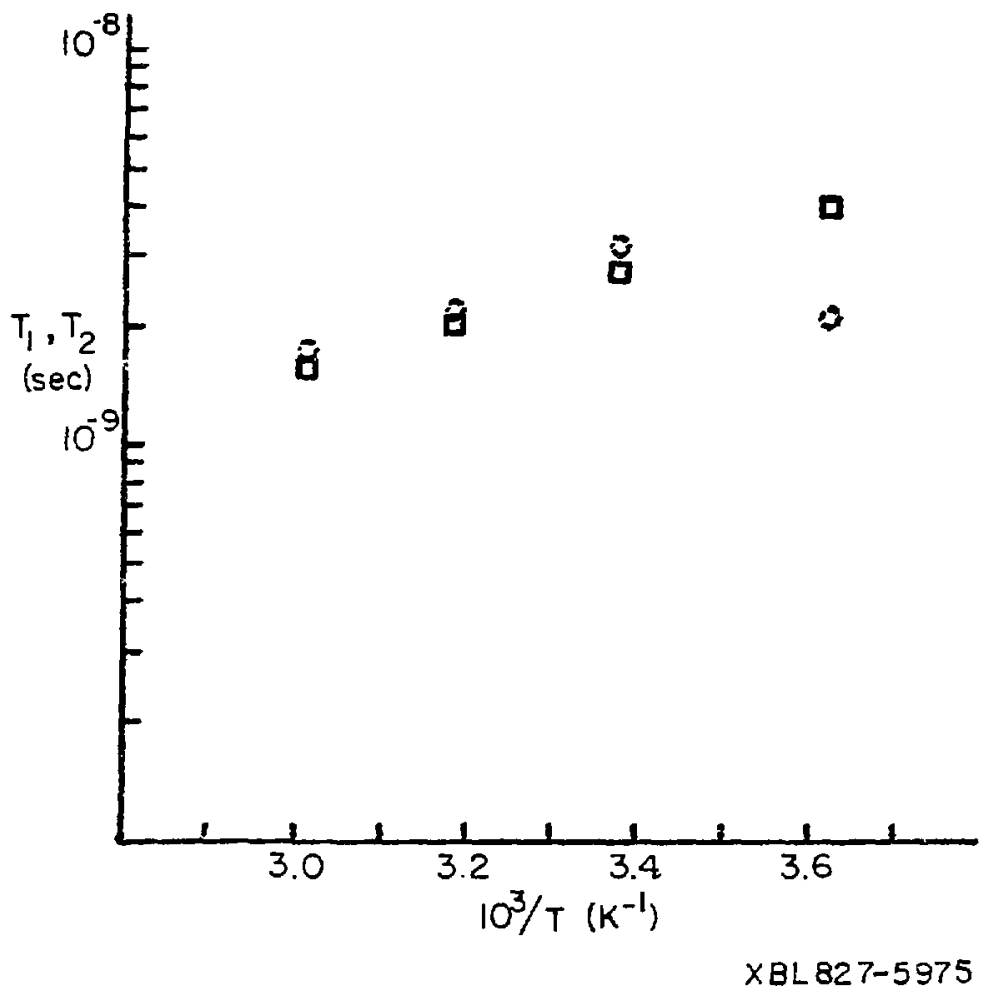

Fig. 12. $T_{1}$ and $T_{2}$ for the $M_{I}=3 / 21$ ine of $\mathrm{Cu}(\text { en })_{2}\left(\mathrm{H}_{2} \mathrm{O}\right)_{2}^{2+}$. Circles-T 1 determined using $\mathrm{VO}^{2}+$ as a standard. Squares $-T_{2}$ from 7 inewidth measurements of Lewis, Alei and Morgan (1966b). 


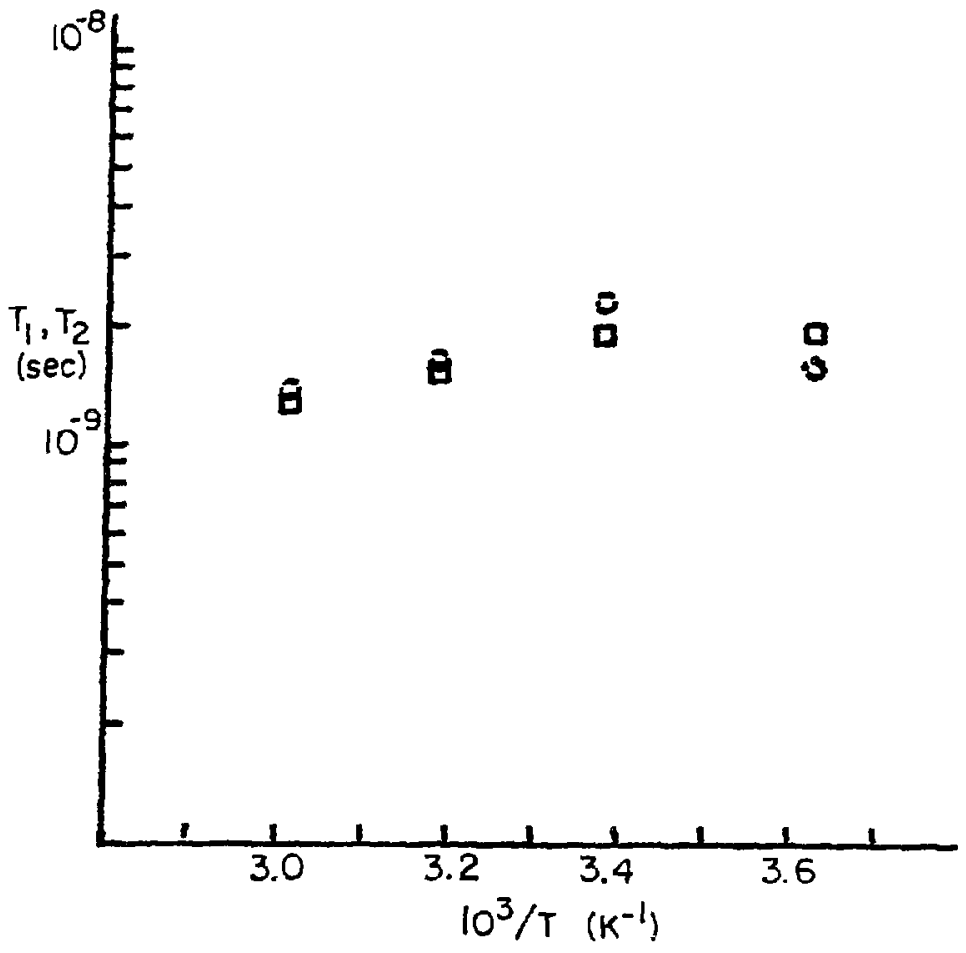

$\times B L 827-5976$

Fig. 13. $T_{1}$ and $T_{2}$ for the $M_{I}=-3 / 2$ line of Cu(en $)_{2}\left(\mathrm{H}_{2} \mathrm{O}\right)_{2}^{2+}$ Circles-T deternined using $\mathrm{V}^{2+}$ as a standard. Squares-T2 from linewidth measurements of Lewis, Alei and Morgan (1966b). 
The measurements at $3^{\circ} \mathrm{C}$ are dissappointing since this is where $T_{1 \mathrm{e}}$ is expected to differ from $T_{2 e}$, especially for the $M_{I}=-3 / 2$ line. For both the $M_{I}=-3 / 2$ and $M_{1}=3 / 2$ line the measured $T_{1 e}$ is less than $\mathrm{T}_{2 \mathrm{e}}$ by much more than experimental uncertainty. This is of course impossible. This discrepancy is surely due to a systematic error. It is partially due to improper matching of the nuclear spin-lattice relaxation times of the $\mathrm{VO}^{2+}$ and $\mathrm{Cu}\left(\mathrm{en}_{2}\left(\mathrm{H}_{2} \mathrm{O}\right)_{2}^{2+}\right.$ solutions, but it is unlikely that this is the only source of this large error. As a check, $\mathrm{Cr}\langle\mathrm{CN})_{6}^{3-}$ ion was also tried as a standard. The relaxation of $\mathrm{Cr}(\mathrm{CN})_{6}^{3-}$ is discussed in detail 1 ater in this work. For $\mathrm{Cr}\langle\mathrm{CN})_{6}^{3-}$ it proves reasonable to assume $\mathrm{T}_{1 \mathrm{e}}=\mathrm{T}_{2 \mathrm{e}}$ at $\mathrm{X}$-band. Linewidth measurements for $\mathrm{Cr}(\mathrm{CN})_{6}^{3-}$ were made by Hayes (1961). The $\mathrm{T}_{1 \mathrm{e}}$ 's using $\mathrm{Cr}(\mathrm{CN})_{6}^{3-}$ as a standard are compared with the $T_{2 e}$ 's in Figs. 14 and 15 . These measurements compare favorably with those using $\mathrm{VO}^{2+}$ as a standard at room temperature and above. These $3^{\circ}$ measurements are more in line with theoretical predictions and the linewidth data of Lewis, Alei and Morgan. Within experimental error $\mathrm{T}_{1 \mathrm{l}}=\mathrm{T}_{2 \mathrm{e}}$ for the $M_{I}=3 / 2$ line, while $T_{1 e}$ is clearly greater than $T_{2 e}$ by more than the experimental uncertainly for the $M_{1}=-3 / 2$ line. At $3^{\circ}$ the tumbling mechanism contributes much more to $T_{2 e}$ than to $T_{1 e}$ for the $M_{I}=-3 / 2$ line.

Figure 16 shows a plot of $1 / T_{1 e} v s T / n$, after $1 / T$ le has been corrected for tumbling. If spin-rotation were the only remaining relaxation mechanism, this plist should extrapolate to zero at $\mathrm{T} / \mathrm{n}=$ 0 . This is not the case. If the slope of the line drawn through the 


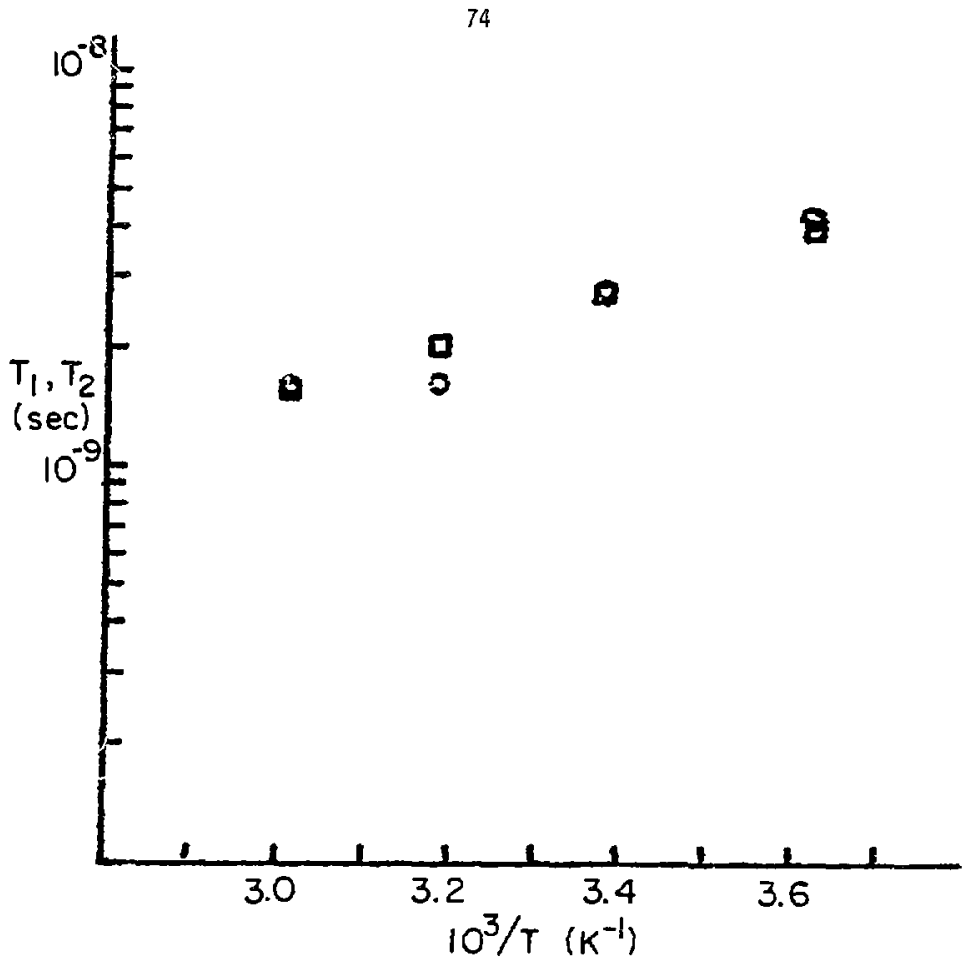

XEL 827-5977

Fig. 14. $T_{1}$ and $T_{2}$ for the $M_{I}=3 / 2$ line of $\mathrm{Cu}(\mathrm{en})_{2}\left(\mathrm{H}_{2} \mathrm{O}\right)_{2}^{2+}$. itircles- $\mathrm{T}_{1}$ determined using $\mathrm{Cr}(\mathrm{CN}){ }_{6}^{3-}$ as a standard. Squares- $\mathrm{T}_{2}$ as in Fig. 12. 


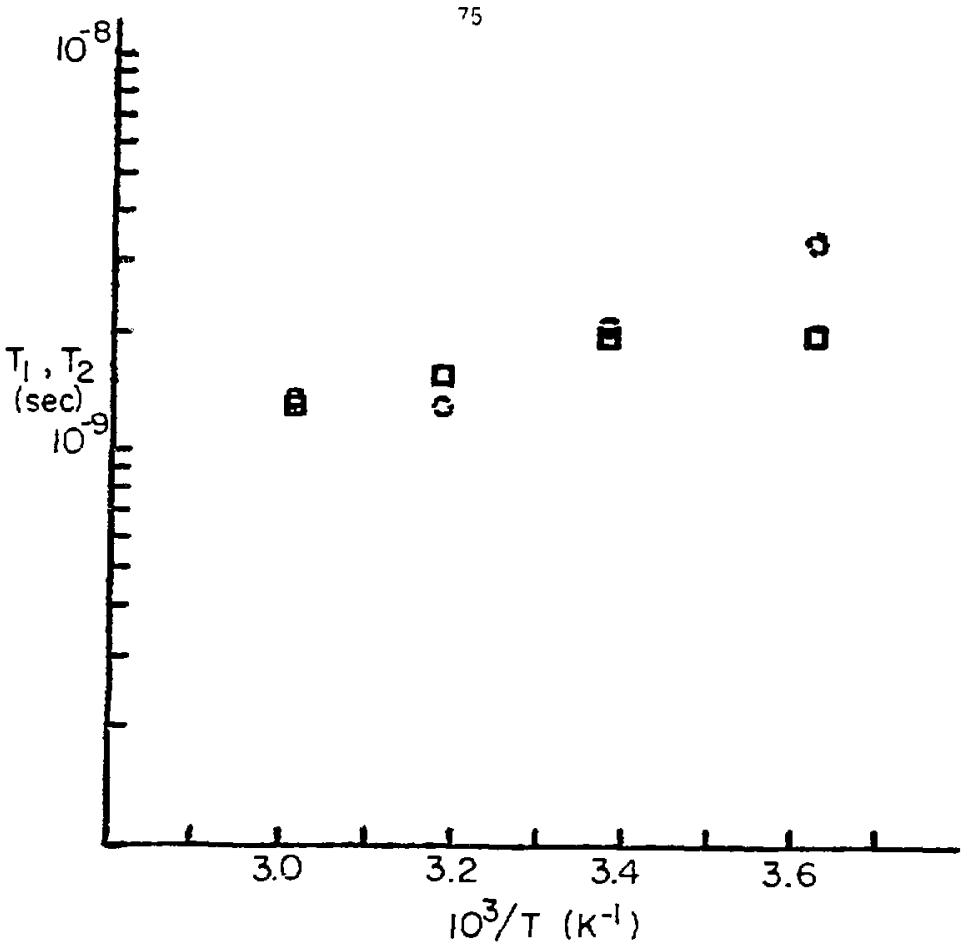

$X B L 827-5978$

Fig. 15. $\mathrm{T}_{1}$ and $\mathrm{T}_{2}$ for the $\mathrm{H}_{1}=-3 / 2$ 1 ine of $\mathrm{Cu}(\mathrm{en})_{2}\left(\mathrm{H}_{2} \mathrm{O}\right)_{2}^{2+}$. Circles-T, determined using $\mathrm{Cr}(\mathrm{CN}) 3-$ as a standard. Squares-T2 as in Fig. 13. 


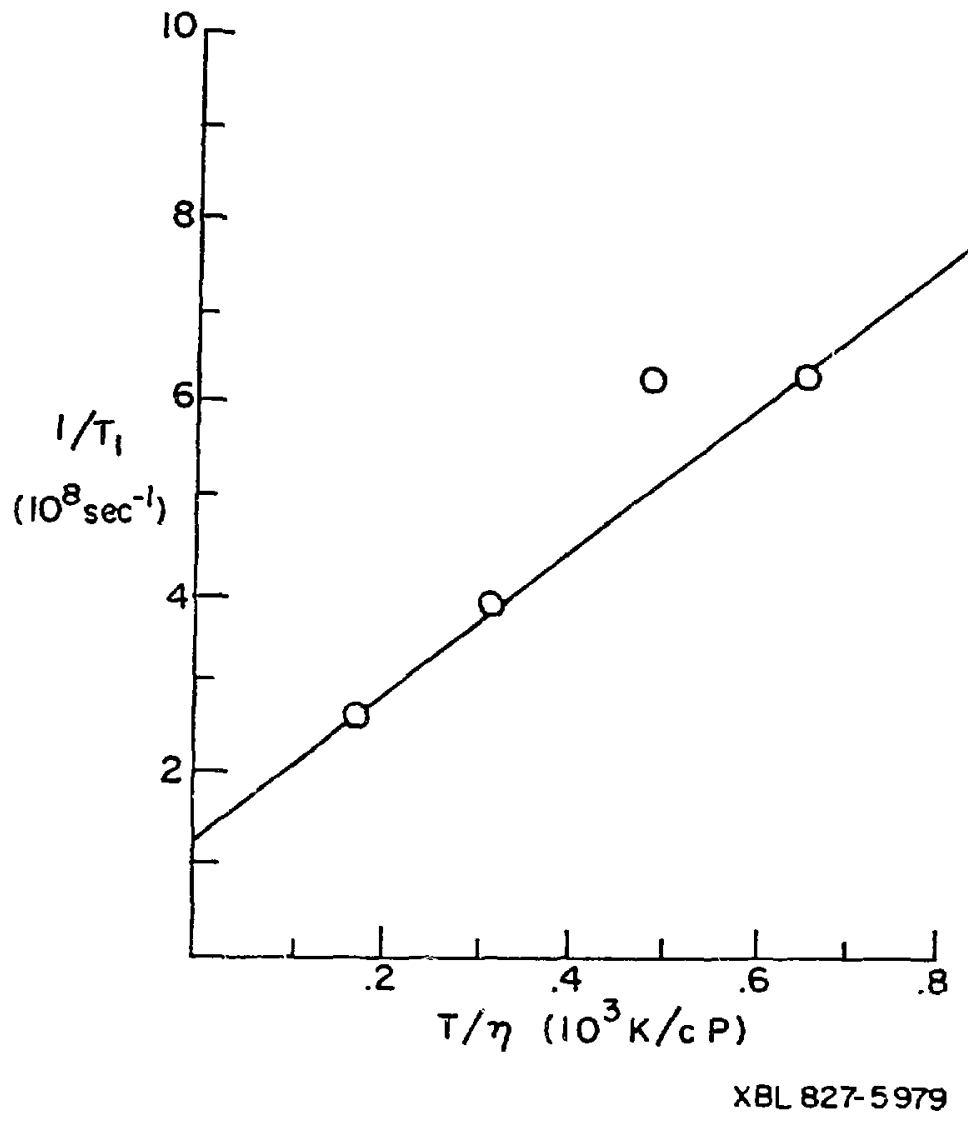

Fig. 16. $1 / T_{1}$ vs $\mathrm{T} / \mathrm{T}$ for $\mathrm{Cu}(\mathrm{en})_{2}\left(\mathrm{H}_{2} \mathrm{O}\right)_{2}^{2+}$. 
points is used to calculate a hydrodynamic radius the resulting tumbling correlation time at $25^{\circ} \mathrm{C}$ is found to be $2.1 \times 10^{-11} \mathrm{sec}$. This agrees better with the correlation time determined from proton relaxation measurements (Morgan, Murphy and Cox, 1959) than if all of the relaxation were considered to be from sp in rotation. Obtaining the hydrodynamic radius in this manner is probably not correct, however. The non-zero intercept indicates that there is another relaxation mechanism in operation besides spin-rotation. Surely this mechanism is not temperature independent, so a proper determination of the hydrodynamic radius wouls. require subtracting the effects of this mechanism first. Lewis, Alei and Morgan did not consider any other relaxation inechanisms for this ion, but in similar work on

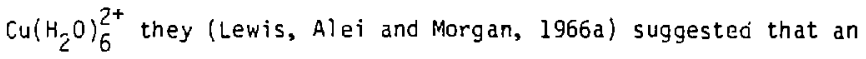
additional mechanism cauld be a Van Vleck Raman relaxation process. Calculations by kivelson (1966) however, indicate that this mechanism should be nealigible in liquids. Elucidation of the additional mechanism or mechanisms might be possi "e with the aid of more precise linewidth measurements.

The results of the ONP measurements, are in reasonable agreement with what would be predicted from theory and the linewidth measurements. The agreement is not, however, as good as would be expected considering the precision with which the DNP enhancements were measured. It toes appear that roughiy one significant figure $c$ an be obtained from DNP $\mathrm{T}_{1 e}$ measurements. The agreement can be improved by making small corrections in the correlation times used in the o/p 
correlations. It seems that the o/p correction is a large contributor to the uncertainty in the $\mathrm{T}_{1 \mathrm{e}}$ values determined from DNP measurements.

C. Manganous Ion

1. Introduction

Manganous ion, $\mathrm{Mn}^{2+}$ is a (3d $)^{5}$ ion. In a weak octahedral crystal field the ground state is the totally symmetric ${ }^{6} A_{1 g}$ state. Because of the absence of low lying orbitally excited states ${ }^{6}{ }_{19}$ ions usually have observable EPR spectra in solution. Tinkham, weinstein, and Kip (1951) were the first to observe the EPR spectrum of $\mathrm{Mn}_{\left(\mathrm{H}_{2} \mathrm{O}\right)_{6}^{2+}}$ in sulutions dilute enough to resolve the $\operatorname{six}^{55} \mathrm{Mn}(\mathrm{I}=5 / 2$, 100 percent ajundant) hyperf ine lines. The six lines have different widths. At first this would suggest a McConnell (1956) tumbling contribution to the 1 inewidth. However, both the $g$ and $A$ tensors are isotropic for the ${ }^{6} \mathrm{~A}_{1 \mathrm{~g}}$ state. This rules out such a tumbling mechanism for this ion.

The spectrum of the $S=5 / 2, I=5 / 2$ ion should consist of thirty transitions allowed by the $\Delta M_{S}=1, \Delta M_{1}=0$ selection rule. The Hamiltonian for the system in solution is $\pi=g B H S=+A S I$. To $f$ irst-wiler the energy of each of the five $\Delta M_{S}=1$ iransitions is $\mathrm{gBH}+\mathrm{AM}_{\mathrm{I}}$, which is independent of $\mathrm{M}_{S}$. Thus each hyperfine line would consist of a sum of five lines at the same field, one for each $\Delta M_{s}=1$ transition. In second and higher orders, however, the hyperfine interaction $S p l i t s$ apart the $\Delta M_{S}=1$ transitions. Each of the six $\Delta M_{I}=0$ hyperfine lines consists of five $\Delta M_{S}=1$ transitions which are split by the higher order hyperfine interaction. They are un- 
resolved because the splitting is less than the linewidths of the $l$ ines. The amount of $s p l i t t i n g$ depends on $M_{I}$, therefore each of the hyperfine lines experiences a different amount of inhomogeneous broadening. This situation tends to complicate both the experimental and theoretical interpretation of the relaxation of $\mathrm{Mn}\left(\mathrm{H}_{2} \mathrm{O}\right)_{6}^{2+}$.

Most models of relaxation for $\mathrm{Mn}^{2+}$ in solution consider the dominant mechanism to be fluctuations in an instantaneous quadratic zero-field splitting. Most other mechanisms can be neglected. Tumbling has already been discounted. The $\mathrm{g}$ value for $\mathrm{Mr}^{2+}$ is very close to the free electron value, so spin-rotation is not likely as a source of relaxation. The octahederal $5=5 / 2$ ions have quartic zero-f ield terms in their spin Hamiltonian which are given by (Coster and Statz, 1959)

$$
\mathscr{C H}=\frac{a}{6}\left[s_{a}^{4}+s_{b}^{4}+s_{c}^{4}-\frac{1}{5} s(s+1)\left(3 s^{2}+3 s-1\right)\right] .
$$

$S_{z}, S_{b}$, and $S_{C}$ represent sp in operators in a molecular coordinate system. The fine structure parameter, $a$, is a function of the crystal field potential. Rotational modulation of this Hamiltonian can induce relaxation, but Luckhurst (1972) has calculated that its contribution should be negligible for $\mathrm{Mn}^{2+}$. This leaves fluctuations of the quadratic zero-field splitting as the most likely mechanism of relaxation.

Much of the theoretical and experimental work pertaining to $\mathrm{Mn}^{2+}$ has been reviewed by Burlamacchi et al. (1978). A faw examples of 
previous work will be discussed here to provide a proper background for the discussion of ONP measurements on $\mathrm{Mn}\left(\mathrm{H}_{2} \mathrm{O}\right)_{6}^{2+}$.

Garrett and Morgan (1966) used Redfield (1957) theory to calculate 1 inewidths for $\mathrm{Mn}^{2+}$. They assumed that relaxation was due to instantaneous fluctua:ions in the quadratic zero-field splitting. They calculated relaxation coefficients $R_{K K^{\prime} \lambda \lambda}$, according to Eq. (2.12). They neglected of $f$-diagonal elements in the relaxation matrix. This treatment led to the conclusion that there were three distinct Tle's and three $\mathrm{T}_{2 \mathrm{e}} \mathrm{s}^{\mathrm{s}}$ for $\mathrm{Mn}^{2^{+}}$, even in the case of extreme narrowing. They found that in the $c$ ase of extreme narrowing

$$
\begin{aligned}
& {\left[\frac{1}{T_{2}}\right]_{3 / 2,5 / 2}=\left[\frac{1}{T_{2}}\right]_{-5 / 2,-3 / 2}=2.14 \mathrm{Z}(0)} \\
& {\left[\frac{1}{T_{2}}\right]_{1 / 2,3 / 2}=\left[\frac{1}{T_{2}}\right]_{-3 / 2,-1 / 2}=1.892(0)} \\
& {\left[\frac{1}{T_{2}}\right]_{-1 / 2,1 / 2}=1.53 z(0)}
\end{aligned}
$$

where

$$
Z(0)=\frac{32}{5}\left\langle D^{2}\right\rangle
$$

and where $\left\langle D^{2}\right\rangle$ is the average of the square of the ZFS tensor. Using Tine positions calculated from perturbation theory (Hurd, Sachs, and Hershberger, 1954) and the relative linewidth information, they were able to simulate the experimental lineshape with moderate success. 
Levanan and Luz (1968) found that it was not possible to fit the most inhomogeneously broadened lines of $\mathrm{Mn}^{2+}$ using the methods of Garrett and Morgan. They felt that this was due to the separated line approximation used by Garrett and Morgan in developing the ir theory. Rubinstein, Barum and Luz (1971) did the relaxation calculation assuming that each hyperfine Iine consisted of five superimposed lines. Under these conditions it is permissible to diagonalize the relaxation matrix formed from Eq. (2.12). They found that only a single $T_{1 e}$ and $T_{2 e}$ were important over a wide range of $\omega_{T e^{*}}$ From a consideration of proton spin-spin and spin-lattice relaxation times measured from 10-100 $\mathrm{MHz}, T_{1 \mathrm{e}}$ could be determined and $\mathrm{s}^{2}$ and could be estimated. The parameter a is given by Eq. (2.17). They found $\tau=3.2 \times 10^{-12} \mathrm{~s}$ at $30^{\circ} \mathrm{C}$ and $\Delta=0.014 \mathrm{~cm}^{-1}$. However, when these numbers were used to calculate the $x$-band linewidth, the value calculated was found to underestimate the linewidth by a factor of two.

Luckhurst and Pedulli (1971) felt that the failure of Garrett and Morgan and Rubinstein, Barum and Luz to properly predict lineshapes of $\mathrm{Mr}^{2+}$ spectra was due to improper treatment of the overlapping 1 ines. since the five transitions of each $\mathrm{Mn}^{2+}$ hyperfine line were neither superimposed nor well separated, they felt ari intermediate treatment of the problem was necessary. Luckhurst and Pedulli used a more general theory of magnetic resonance 1 ineshapes (Binch, 1968) to simulate $\mathrm{Mn}^{2+}$ spectra. This method involves calculation of the lineshape function, $L(\omega)$, where

$$
L(\omega) \quad-\operatorname{Re}\left[\stackrel{\mathrm{S}}{\left.\mathrm{M}_{0}^{-1} \mathrm{~g}\right]}\right]
$$


$\xi_{-}$is the vector composed of matrix elements $\left\langle\left. k\right|_{S} \mid K^{\prime}\right\rangle$, of $S_{-}$and $\sigma$ is given by

$$
\underline{c}=\left\langle K\left|S_{-}\right| K^{\prime}\right\rangle\left(0_{K K}-o_{K} K^{\prime}\right)
$$

where ${ } K_{K}-\rho_{K^{\prime} K^{\prime}}$ are the difference in the diagoral elements of the density matrix for the system. The matrix $M_{0}$ is given by

$$
M_{O_{K K^{\prime}, \lambda \lambda}}=i\left(\omega_{K K^{\prime}}-\omega\right) \delta_{K \lambda} \delta_{K^{\prime} \lambda}+R_{K K^{\prime}, \lambda \lambda^{\prime}},
$$

where $R_{K K^{\prime}, \therefore \lambda}$ is an element of the relaxation matrix calculated from Eq. (2.12) and $\omega_{K K_{1}}$ is the resonant frequency for the transition from $k^{\prime} \rightarrow k$. When all the $\omega_{k K} \cdot$ 's are equal this method is equivalent to that employed by Rubinstein, Borum and Luz (1971). When the lines are well separated it reduces to the method used by Garrett and Morgan (1966). Luckhurst and Pedulli caiculated the relaxation matrix for fluctuations in the quadratic term of the zerorifield splitting. They were able to simulate the spectrum of $\mathrm{Mn}_{2}\left(\mathrm{H}_{2} \mathrm{O}\right)_{6}^{2+}$ at $90^{\circ} \mathrm{C}$ with excellent agreement using $\Delta=690 \mathrm{MHz}$ and $\tau=1.18 \times 10^{-12} \mathrm{~s}$. They also determined the resonant frequencies by numerical diagonalization of the static Hamiltonian matrix, which probably aided in the excellent agreement between experiment and simulation. They felt that this agreement confirmed that the major relaxation process for $\mathrm{Mn}^{2+}$ is fluctuation in the quadratic zero-field splitting terms. 
Levanan and Luz (1968) found that it was not possible to fit the most inhomogeneously broadened lines of $\mathrm{Mn}^{2+}$ using the methods of Garrett and Morgan. They felt that this was due to the separated line approximation used by Garrett and Morgan in developing their theory. Rubinstein, Barum and Luz (1971) did the relaxation calculation assuming that each hyperfine line consisted of five superimposed lines. Under these conditions $i t$ is permissible to diagonalize the relaxation matrix formed from Eq. (2.12). They found that only a single $i_{\text {le }}$ and $T_{2 e}$ were important over a wice range of $\omega_{\tau c^{*}}$. From a consideration of proton spin-spin and spin-lattice relaxation times measured from 10-100 $\mathrm{MHz}$, Tle could be determined and $\Delta^{2}$ and could be estimated. The parameter $\Delta$ is given by Eq. $\left\{2.17\right.$ ). They found $\tau=3.2 \times 10^{-12} \mathrm{~s}$ at $30^{\circ} \mathrm{C}$ and $\Delta=0.014 \mathrm{~cm}^{-1}$. However, when these numbers were used to calculate the $x$-band linewidth, the value calculated was found to underestimate the linewidth by a factor of two.

Luckhurst and Pedulli (1971) felt that the failure of Garrett and Morgan and Rubinstein, Barum and Luz to properly predict line:iapes of $\mathrm{Mn}^{2+}$ spectra was due to improper treatment of the overlapping 1 ines. Since the five transitions of each $\mathrm{Mn}^{2+}$ hyperfine line were neither superimposed nor well separated, they felt an intermediate treatment of the problem was necessary. Luckhurst and Pedulli used a more general theory of magnetic resonance 1 ineshapes (Binch, 1968) to simulate $\mathrm{Mn}^{2+}$ spectra. This method involves calculation of the lineshape function, $L(w)$, where

$$
L(\omega) \quad-\operatorname{Re}\left[\stackrel{S}{M}_{\rightarrow}^{-1} g\right]
$$


$S_{-}$is the vector composed of matrix elements $\left\langle\left.\kappa\right|_{S_{-}-} \mid \kappa^{\prime}\right\rangle$, of $S_{-}$and 0 is given by

$$
\underline{a}=\left\langle k\left|S_{-}\right| k^{\prime}\right\rangle\left(\rho_{K \kappa}-\rho_{K^{\prime} k^{\prime}}\right)
$$

where $\rho_{K K}-P_{K^{\prime} K^{\prime}}$ are the difference in the diagunal elements of the density matrix for the system. The matrix $M_{0}$ is given by

$$
M_{O_{K K^{\prime}, \lambda \lambda}}=i\left(\omega_{K K^{\prime}}-\omega\right) \delta_{K \lambda} \delta_{K^{\prime} \lambda}+R_{K K^{\prime}, \lambda \lambda^{\prime}},
$$

where $R_{K K^{\prime}, \lambda \lambda}$ is an element of the relaxation matrix calculated from Eq. (2.12) and $w_{K K 1}$ is the resonant frequency for ths transition from $\kappa^{\prime} \rightarrow \kappa$. When all the $\omega_{\kappa \kappa} I^{\prime} s$ are equal this method is equivalent to that employed by Rubinstein, Borum and Luz (1971). When the Tines are well separated it reduces to the method used by Garrett and Morgan (1966). Luckhurst and Pedulli calculated the relaxation matrix for fluctuations in the quadratic term of the zero-field splitting. They were able to simulate the spectrum of $\mathrm{Mn}\left(\mathrm{H}_{2} \mathrm{O}\right)_{6}^{2+}$ at $90^{\circ} \mathrm{C}$ with excellent agreement using $\Delta=690 \mathrm{MHz}$ and $\mathrm{\tau}=1.18 \times 10^{-12} \mathrm{~s}$. They also determined the resonant frequencies by numerical diagonalization of the static Hamiltonian matrix, which probably aided in the excellent agreement between experiment and simulation. They felt that this agreement conf irmed that the major relaxation process for $\mathrm{Mn}^{2+}$ is fluctuation in the quadratic zero-field splitting terms. 
$\mathrm{Mn}^{2+}$ ion was also the subject of early DNP work. Measurements in a field of $5 \mathrm{G}$ on an aqueous solistion of $\mathrm{MnCl}_{2}$ (Codrington and Bloembergen, 1958) showed an increase in the intensity of the proton MR signal when the electronic transition was saturated. This was an indication of the dominance of the scalar contribution to the spin lattice relaxation time of the protons at 5 G. Kul'beda (1964) measured the temperature dependence of the DNP of aqueous $\mathrm{Mn}^{2+}$ at $80 \mathrm{G}$. He found that the sign of the enhanceme th changed at $60^{\circ} \mathrm{C}$, indicating a switch in the importance of the electran-nuclear coupling from dipolar te scalar.

At the magnetic field used in this work, UNP is dominated by the Iipoliar interaction. Proper treatment of DNP data should lead to reasonable estimates of $\mathrm{T}_{\mathrm{le}}$ for $\mathrm{Mn}_{n}\left(\mathrm{H}_{2} \mathrm{O}\right)_{6}^{2+}$. Since previous work on the lineshape of $\mathrm{Mn}^{2+}$ seems to conf irm that the dominant mechanism of relaxation is fluctuations in the quadratic zero-fielo splitting, meascirements of $T_{l e}$ are not likely to contribute a great deal more to tha understanding of the mechanism. However, $T_{1}$ measurements should conf im that relaxation occurs through a mechanism with a correlation time short enough that $\omega \tau_{c} \ll 1$. If $r_{c}$ is $-10^{-12} \sec$ then at $x-b$ and with $\omega_{e} \sim 6 \times 10^{10}$ it should be found that $T_{1 e}=T_{2 e}$. The DNP results should also be interesting as a direct measurement of $T_{\text {le }}$ to compare with the results of NMR relaxacion measurements on aqueous $\mathrm{Mn}^{2+}$ solutions.

\section{Experimental}

So lutions of $\mathrm{Mn}^{2+}$ ions were prepared from $\mathrm{Mn}\left(\mathrm{ClO}_{4}\right)_{2} \cdot 6 \mathrm{H}_{2} \mathrm{O}$ (G. Frederic Smith). The perchlarate salt was used to avoid large 
anion effects on the $\mathrm{Mn}^{2+}$ relaxation (Hayes and Myers, 1964). The concentration was adjusted aciording to proton $\mathrm{T}_{1}$ measurements (Morgan and Nolle, 1959) to insure that nuclear $T_{1}$ values of the $\mathrm{Mn}^{2+}$ solution were approximately the same as the nuclear $T_{1}$ values of the $\mathrm{VO}^{2+}$ reference solution. DNP measurements were made in the flowing sample spectrometer described earlier with $15 \mathrm{~W}$ of incident microwave power.

\section{Results and Discussion}

Linecenter enhancements were measured for the $M_{I}=1 / 21$ ine of $\mathrm{Mn}\left(\mathrm{H}_{2} \mathrm{O}\right)_{6}^{2^{+}}$at $3,23,41$, and $59^{\circ} \mathrm{C}$. These enhancements are $i$ isted in Táble il. The vaiues given have been corrected for overlap of adjacent hyperfine lines. The values of $T_{\text {je }}$ calculated from these enhancements are also given in Tade 11. The calculation was made according to Eq. (5.17) with the proper correction factor for the number of $\mathrm{Mn}^{2+}$ hyperfine lines. The correlation time for the o/p corrections were based on the value from proton NMR relaxation measurements (Morgan and Nolle, 1959), $3.1 \times 10^{-11} \mathrm{sec}$ at $27^{\circ} \mathrm{C}$. Linewidths for $\mathrm{Mn}^{2+}$ were interpolated from measurements by Hayes (1961). This treatment of the data assumes that the $M_{I}=1 / 2$ line is a single lorentzian, which the preceding discussion made clear is not quite the case. Howtier, for this line perturbation theory predicts that the largest separation between linecenters of the unresolved lines is $5.4 \mathrm{G}$. Even at $59^{\circ} \mathrm{C}$ the peak-to-peak linewidth is $12.2 \mathrm{G}$, , more than twice the splitting. At lower temperatures the linewidths 2 e larger. There is little to be gained from simulating lineshapes of enhancement 
Table 11. DNP Results for $\mathrm{Mn}^{2+}$

\begin{tabular}{llll}
\hline$T^{\circ} \mathrm{C}$ & $\mathrm{E}$ & $\Delta \mathrm{H}_{\mathrm{pp}}^{\mathrm{a}}(\mathrm{G})$ & $\mathrm{T}_{\mathrm{le}}^{\mathrm{b}}(\mathrm{sec})$ \\
\hline $3^{\circ}$ & 0.049 & 35 & $1.7 \times 10^{-9}$ \\
23 & 0.136 & 22.2 & $2.8 \times 10^{-9}$ \\
41 & 0.464 & 16.6 & $5.0 \times 10^{-9}$ \\
59 & 0.909 & 12.2 & $5.9 \times 10^{-9}$ \\
\hline a From Hayes (1961) & & \\
b From DNP (this work) & &
\end{tabular}


spectra at these temperatures. The experimental precision does not permit the assignment of separate $T_{l e}$ 's for lines which are this poorly resolved. The treatment of the $M_{I}=1 / 21$ ine as a single line is the best that can be done with the data at hand.

The calculated $\mathrm{T}_{1 e}$ values are compared in Fig. 17 with $\mathrm{T}_{2 e}$ values which were calculated from the 7 inewidth under the same assumption of a single lorentzian line. The valtes of $\mathrm{T}_{1 e}$ and $\mathrm{T}_{2 e}$ agree reasonably well with each other. This is not surprising since they were determined using the same assumptions, and at $X-b$ and $\omega \tau=0.03$ for $t=3.2 \times 10^{-12} \mathrm{sec}$ (Rubinstein, Barum, and Luz, 1971).

The measured $T_{1}$ at $23^{\circ}$, which is $2.8 \times 10^{\circ} \mathrm{sec}$, does not agree well with the value, $9.4 \times 10^{-9} \mathrm{sec}$ determined by Rubinstein, Barum and Luz (1971) from proton relaxation data at $30^{\circ} \mathrm{C}$. There is reasonable agreement between the value measured from DNP and the value determined from best fits to temperature dependent relaxation data for protons, $3.5 \times 10^{-9} \mathrm{sec}$ (Bi vembergen and Morgan, 1961) and ${ }^{17} 0$, $3.8 \times 10^{-9} \sec$ (Swift and Connick, 1962). The value determined by Rubinstein et al. is based on a measurement of the scalar coupling constant between the electrons and protons from the chemical shift of the protons in $\mathrm{Mn}^{2+}$ solution (Luz and Shulman, 1965). The value for the superhyperfine coupling constant which they used, $6.2 \times 10^{5} \mathrm{~Hz}$, is less than the values determined from best fits to temperature dependences of proton relaxation in $\mathrm{Mn}^{2+}$ solution $\left(1 \times 10^{6} \mathrm{~Hz}\right.$ by Bloembergen and Morgan, 1961 and $0.70 \pm 0.03 \times 10^{6} \mathrm{~Hz}$ by Sprinz, 1967). Electron-nuclear double resonance measurements on crystals of $\mathrm{La}_{2}(\mathrm{Mg}, \mathrm{Mn})_{3}\left(\mathrm{NO}_{3}\right)_{12} \cdot 24 \mathrm{H}_{2} \mathrm{O}$ (Van Ormondt, et al. 1969), which contains 


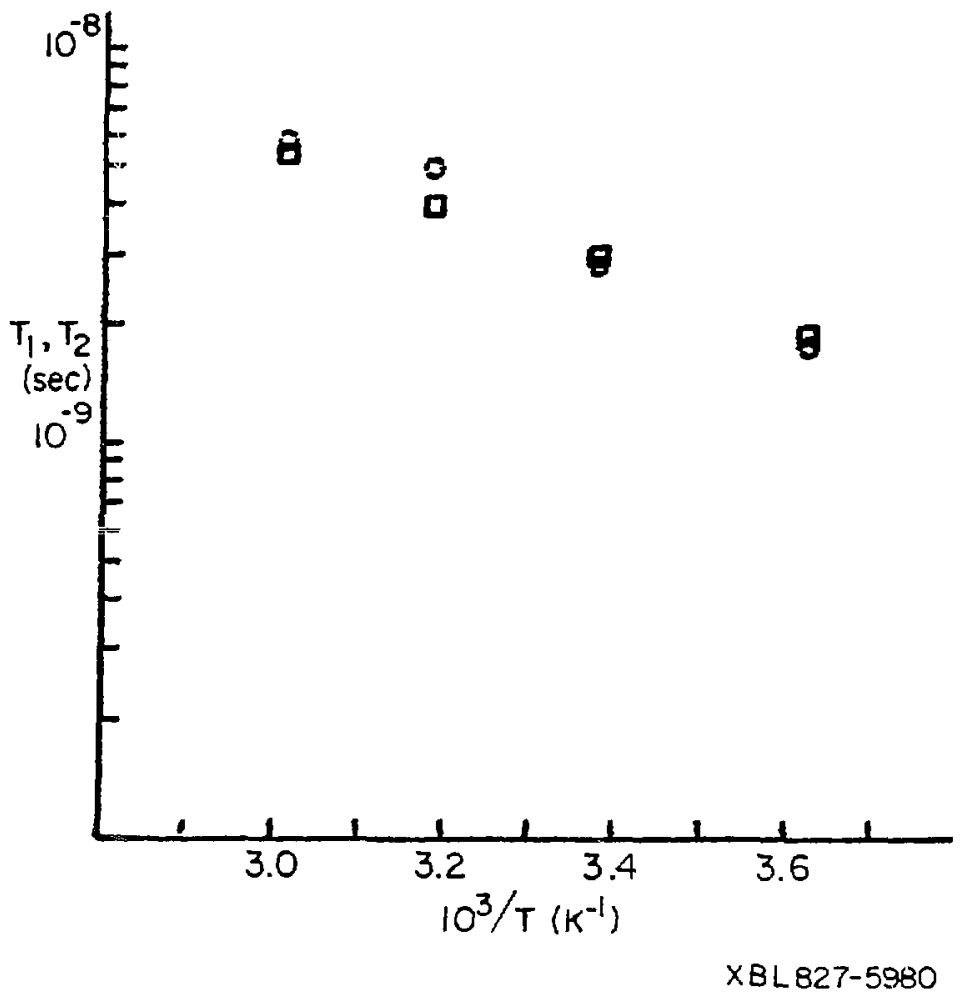

Fig. 17. $T_{1}$ and $T_{2}$ for $\mathrm{Mn}^{2+}$. Circies-T, from ONP; Scuares-T2 from linewidth measurements (Hayes, 1961). 
the $\operatorname{Mn}\left(\mathrm{H}_{2} \mathrm{O}\right)_{0}^{2+}$ entity, give a superhyperf ine coupling constant of $0.80 \times 10^{6} \mathrm{~Hz}$. T $T_{1}$ was determined by Rubinitein et al. from the expression

$$
\frac{1}{T_{2 c}}=\frac{A^{2} S(S+1) r_{e}}{3}
$$

where $T_{2 c}$ is the proton spin-sp in relaxation time corrected for the dipolar contribution. $A$ is the electron-proton superhyperf ine coupling constant and $S$ is the total electronic spin. The electron spin-exchange correlation time, $\tau_{e}$ is given by

$$
\frac{1}{\tau_{e}}=\frac{1}{T_{h}}+\frac{1}{T_{l e}} \text {, }
$$

where $\tau_{h}$ is the lifetime of a proton in the first coordination sphere of $\mathrm{Mn}^{2+}$. The measured value of $\mathrm{T}_{1 \mathrm{l}}$ determined from this method viries with $A^{-2}$. If the value of $A$ were $0.80 \times 10^{6} \mathrm{~Hz}$ then the value of $T_{l e}$ calculated in this manner would be $4.0 \times 10^{-9} \mathrm{sec}$, which is much closer to the value determined from direct 1 inewidth measurements and the ONP measurements of this work. Thus, if allowance for possible uncertainty in the value of $A$ is made, the values of $T_{1 e}$ determined from MR measurements do not differ too significantly from those using DNP and treating the $M_{I}=1 / 2$ line as a single 1 ine. It appears that the more sophisticated lineshape treatment employed by Luckhurst and Pedulli (1971) is not necessary except at high temperatures where the inhomgenities contribute a significant portion to the linewidth of the $M_{I}=1 / 2$ line. 


\section{Moving Sample Spectrometer}

\section{A. Introduction}

The flowing samp to ONP spectrometer described earlier has a number of drawbacks to its operation. Most importantly, the flow system requires 150-200 ml of solution, which makes sample preparation inconvenient at best and impossible for expensive chemicais or isotopically substituted material. Al so, tne requirement that enhancements $c$ an only be compared between solutions of approximately the same $\mathrm{T}_{\text {In }}$ makes sample preparation and analysis important and occasionally makes it necessary to extrapolate $T_{\text {In }}$ information from theory when measured $v$ alues at the desired frequency and temperature are not available from the literature. Finally, the resolution of the NMR spectrometer is limited by the jifetime of the flowing sample in',e coil. This is important if DNP on solvents with more than one type of proton is to be observed.

These drawbacks can be overcome by changing to a moving samplc spectrometer. The MMR coil is still located outside of the microwave cavity, but in this case after DNP is induced by a pulse of microwave power, the entire sample tube is moved by a pulse of compressed air. Polarized nuclei from within the cavity are moved to the NMR coil rapidly enjugh to allow observation of the enhancement before it can decay away. Less than $0.2 \mathrm{ml}$ of solution is required for this experimenta! arrangement, allowing DNP experiments on a wider range of materials. The details of the moving sample spectrometer are described in this section. 


\section{B. Samois Arrangement}

A uiagram of the sample arrangement is shown in fig. 18. The samples are sealed in standard melting point tubes of $1.5-1.8 \mathrm{~mm} 0 . d$. Sealing can be accomplished a number of ways. If $h i g h$ temperatures are not reached a simple paraffin plug is acceptable. For higher temperature work tubes $c$ an be sealed by inserting a nylon plug which is coated with epoxy. Tubes can also be sealed by inserting a low viscosity potting resin, Ecoseal W-19 (Emerson and Cuming Inc., Technical Bulletin 2-2-7) with a syringe. All of the stuling methods leave the exterior of the sample tube unchanged in order to allow unimpeded motion of the tube. The sample tube fits inside of a $3 \mathrm{~mm}$ o.d. standard glass tube which runs through a cylindrical $T E_{011}$ $x$-band microwave cavity. This tube arrangement is surrounded by a quartz Dewar through which a temperature controlled gas can be flowed. Temperature is controlled by the feedback system shown in Fig. 19. Temperature $c$ an be monitored by a thermocouple placed down the dewar near the sample. The outer glass tube is sealed to an aluminum tube which is threaded to accept a screw containing the upper. stop wire. The lower stop wire is supported by the tee which also serves as the inlet for the compressed air. The MM coil is wrapped around the $3 \mathrm{~mm}$ tube just outside the microwave cavity. The stops are positioned to enable the zortion of the sample from the center of the cavity to move inside of the MMR coil when the corpressed air is turned an. Sirice the import ant part of the microweve magnetic field distribution is given by Eq. (4.3), $H_{1}^{2}$ is the largest at the center of the cavity. Errors in positioning the stops would result in less 


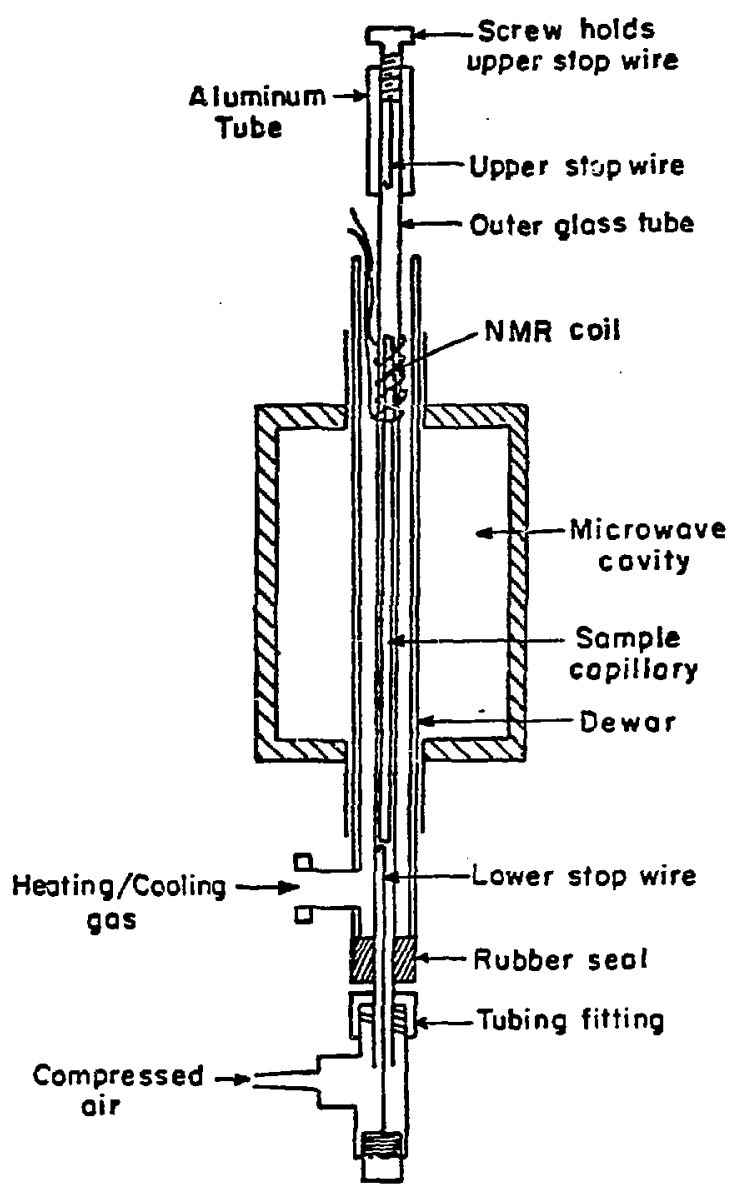

$X B<827-5981$

Fig. 18. The cavity arrangennent for the moving sample tube spectrometer. 
92
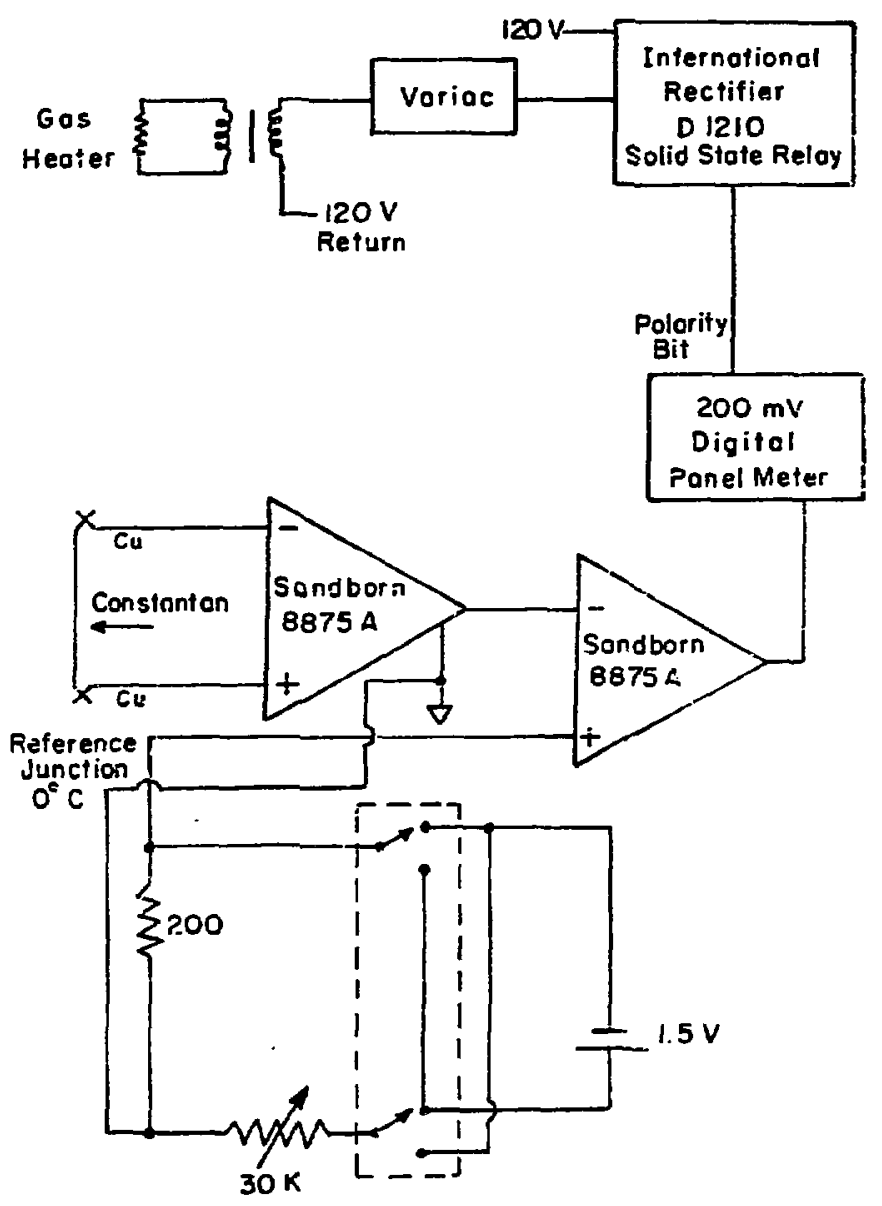

XBL $827-5982$

Fig. 19. A simple temperature controller. 
than optimum enhancements. After the NMR spectrum is acquired, the compressed $a$ ir is turned off and the sample tube is allowed to drop by gravity.

The compressed air is controlled by a small commercial 120V, $60 \mathrm{iz}$ two way solenoid value from Allied Control Company. The valve is opened and closed on computer command through an International Rectifier 01210 solid state relay. The valve vents to the room when it is off, allowing the sample to orop to the lower stop when the compressed air is turned off. The sample tube reaches the upper stop within $40 \mathrm{~ms}$ after the move command is given, when the driving air pressure is 35 psig. About one half of the $40 \mathrm{~ms}$ is due to switching delay in the relay and solenoid, and a pressure propagation delay from the solenoid valve to the sample. The rest of the $40 \mathrm{~ms}$ is spent in the actual motion of the sample tube.

\section{Microwave P!wwer}

The microwave power system is essentially the same as the one used for the flowing sample spectrometer. The V-58C klystron provides drive for the Varian $V A-618 M$ traveling wave tube. The microwaves are pulsed on and off under computer control by the Hewlett-Packard $8735 \mathrm{~B}$ PIN modulator. The microwave frequency and power level can be adjusted by observing incident and reflected power with crystals while the microwaves are pulsed on and off at a low duty cycle to avoid heating the sample excessiveiy.

The cavity is a right circular cylinder of length $2.9 \mathrm{~cm}$ and dianteter $4.9 \mathrm{~cm}$. These dimensions allow a $9 \mathrm{~cm}$ capillary to rest with solution simultaneously in the microwave cavity and the MR coil. 
This makes simultaneous tuning of the microwave and MR system possible without having to move the sample with the compressed air.

\section{MRR Spectrometer}

The requirement that the polarization be measured as soon as possible after the microwaves are turned off makes a pulse spectrometer necessary for this experimental arrangement. The block diagram of the spectrometer constructed for this purpose is shown in Fig. 20.

Radio frequency pulses are generated by switching the output from a General Radio GR-1164A synthesizer using two mini-circuits SRA-1 double-balanced mixers in series. The switching pulses are provided from an Intercontinental Instruments $P G-2$ pulse generator on computer comand. The r.f. pulses are amplified by a 8oonton 230 power amplifier. The receiving coil is made of 10 turns of \#28 wire wrapped around the $3 \mathrm{~mm}$ tube. A set of tuning and matching capacitors allows operation from 1I-17 MHz. The pre-amp is protected by a set of crossed ING14B diodes to ground following a quarter wavelength cable. The pre-amp is isolated from the transmitter by 5 pairs of IN $914 \mathrm{~B}$ crossed diodes in series. The radio frequency amplification of the signal is provided by two Watkins-Johnson thin film amplifiers, followed by a Motorola MHA 320 and an Anzac AM-105. This collection delivers $61 \mathrm{~dB}$ of gain. The signal is mixed with some of the r.f. from the synthesizer in an Hewlett-Packard $10534 \mathrm{~A}$ mixer. The signiel from the mixer is amplified by a LHOO44C to give a few volts of signal with a bandwidth of about $1 \mathrm{KHz}$. Free induction decays are acquired by an Analog Devices ADC1002 12 bit analog to digital converter, which is interf aced to a PDP-8I computer. 


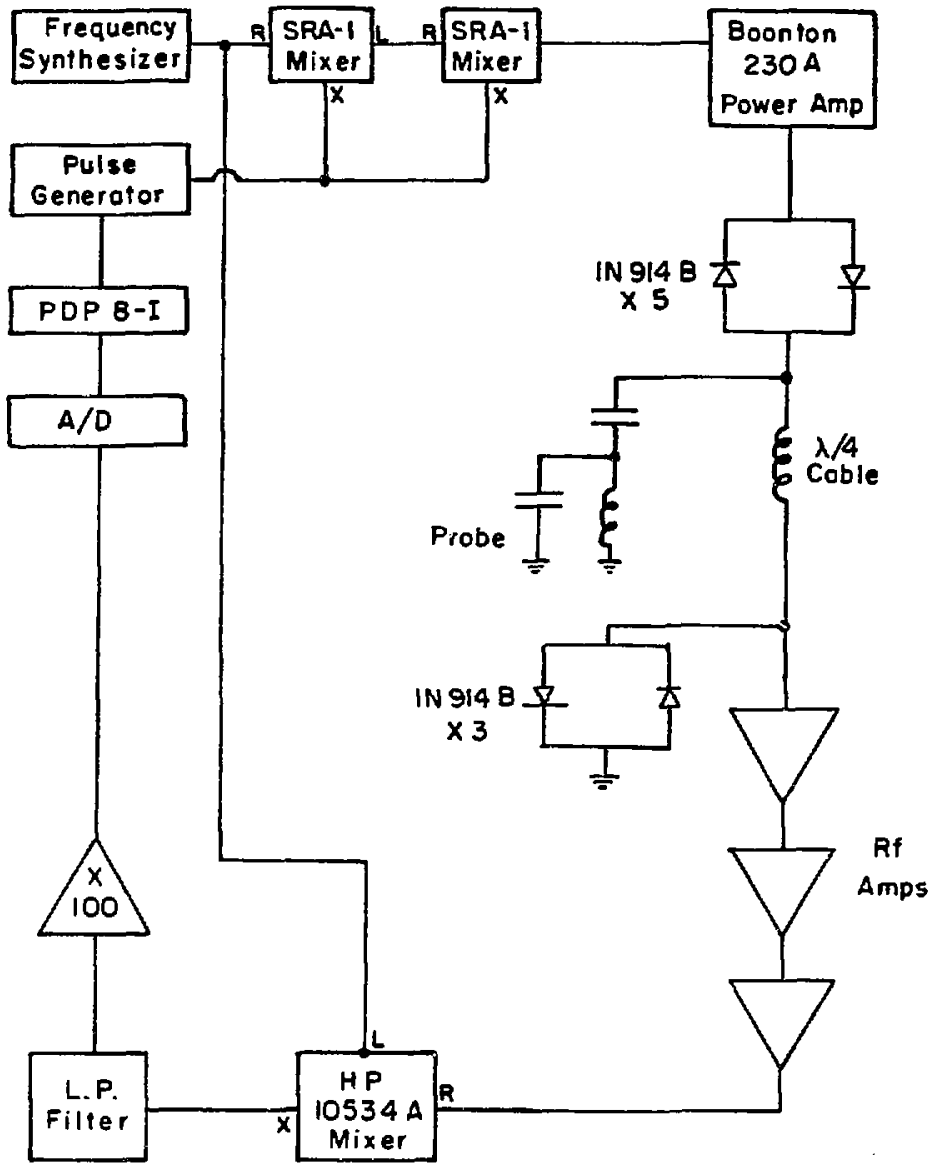

XBL $827-5983$

Fig. 20. Block diagram of the pulse NHR spectrometer used in moving sample tibe DNP measurements. 
The Fourier transforms of the free induction decays are desired to discriminate between signals from non-equivalent nuclei and to preserve phase information in order to tell if enhancements are positive or negative. Sof tware was written to support a commercially available Fast Fourier Transform subroutine (Digita) Equipment Corporation Users Society, 8-143) for the POP-81. Transformed spectra are displayed on a Tektronix 611 storage oscilloscope. The computer is used to calculate and average intensities as was dune in the case of the $\mathrm{CW}$ spectrometer described previously.

The magnet $i:$ field is supplied by the same equipment as the $\mathrm{CW}$ spectrometer. The instability of the magnetic field precludes the averaging of free induction decays, but it is small enough to allow averaging of intensities of successive transformed spectra.

\section{E. Experimental Timing}

Timing for a DNP experiment is controlled by the PDP 81 . The timing for a typical experiment is shown in Fig. 21.

First, the microwaves are turned on. Because it is at least. $20 \mathrm{~ms}$ after the move command is given before the sample begins to move, the move command is given $20 \mathrm{~ms}$ before the microwaves are turned off. A delay of at least $20 \mathrm{~ms}$ more is taken to allow the sample to travel to the upper position. After this delay the radio frequency pulse is applied and the free induction decay is acquired.

Af ter the MMR is acquired the air is turned off and the sampie falls back to its initial position. The computer then computes the Fourier Transfom and signal intensities, and displays the spectrum, This cycle is repeated wi th the microwaves alternately on and off. 


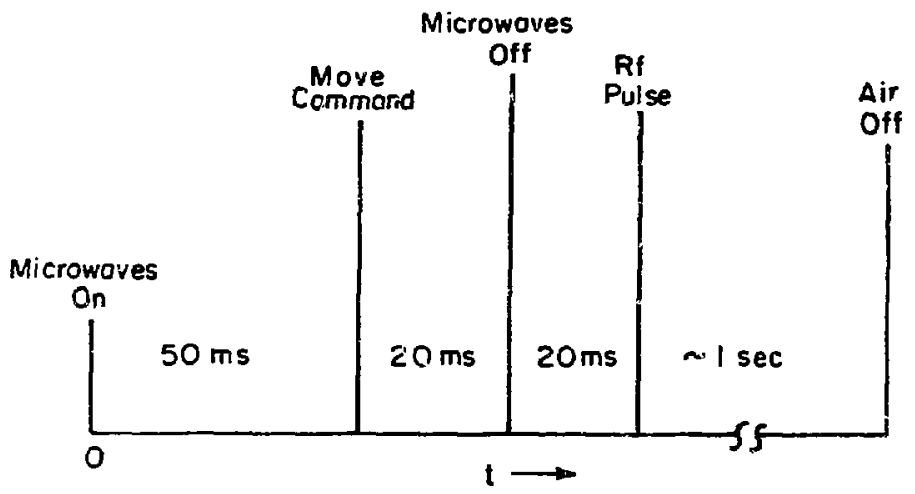

XBL827.5984

Fig. 21. Timing diagram for a typical moving sample tube DNP experiment. 
The microwave pulse length is limited to control the temperature rise, but a $70 \mathrm{~ms}$ pulse still results in approximately $1.5^{\circ} \mathrm{C}$ of temperature rise. The computing time between cycles (3-4 sec to transform and phase 1000 points) allows for some thermal recovery of the sample, but successive cycles are usually limited to five to prevent a lange cumulative temperature rise.

Using the definition of specific enhancement given in Eq. (3.3), the enhancement observed by this technique is

$$
E_{\text {observed }}=E_{\infty}\left(1-e^{-t_{m} / T} \ln \right) e^{-t_{d} T \ln } \text {, }
$$

where $E_{\infty}$ is the enhancement for an inf initely long microwave pulse with no delay before MR measurement, $t_{m}$ is the length of the microwave pulse and $t_{d}$ is the delay before observation of the NMR. Since the length of the microwave pulse is limited by heating effects and the delay is limitad by the transit time of the sampie, $T_{1 n}$ must be adjusted favorably for enhancement to be observed. Usually $\mathrm{T}_{\text {in }}$ can be adjusted by changing the concentration of the paramagnetic species. For $t_{m}=70 \mathrm{~ms}$ and $t_{d}=20 \mathrm{~ms}$ a $T_{1 n}$ of $45 \mathrm{~ms}$ is optimum. By holding the microwave oulse length constant and varying the delay time, $T_{\text {In }}$ may be measured, since then

$$
E_{\text {nbserved }}=E_{n o \text { delay }} e^{-t_{d} / T_{1 n}}
$$


An example of such a measurement is shown in Fig. 22a. When $T_{\text {In }}$ is known, Eq. $(6,1)$ can be used to calculate $E$. Thus as long as enhancement $c$ an be observed with sufficient signal to noise to measure $T_{1 n}$, matching $T_{1 n}$ 's between samples is not necessary, and neither are extrapolations of $T_{\text {In }}$ 's from the literature. Alternatively, the delay time could be held constant and the microwave pulse length varied, to give

$$
E_{\text {observed }}=E_{\text {delay }}\left(1-e^{-t_{m} / T_{1 n}}\right)
$$

as shown in Fig. 22b, but this is less practial since the data from long pulse lengths are likely to be inaccurate from heating effects. F. Estimates of $\left\langle\mathrm{H}_{1}^{2}\right\rangle_{s}$

One of the primary reasons to have a moving sample spectrometer is to keep the MR coil outside of the microwave cavity in order to maximize $\left\langle\mathrm{H}_{1}^{2}\right\rangle_{\mathrm{s}}$. It is interesting to make some estimates of $\left\langle H_{1}^{2}\right\rangle_{s}$ for this experimental arrangement.

Poole (1967) gives expressions for $\left\langle H_{1}^{2}\right\rangle_{s}$ as a function of a number of experimental parameters. Ass:ming that the on $y$ effect of placing the samp:e in the cavity is to lower the quality factor, Q, of the cavity, Poole finds

$$
\left\langle H_{1}^{2}\right\rangle_{S}=2 \times 10^{-3} P_{\omega} Q_{L} n\left(V_{\omega} / V_{L}\right)
$$

where $P_{w}$ is the incident power in watts and $Q_{L}$ is the quality factor of the loaded cavity. $v_{\omega}$ is the volume of the section of 


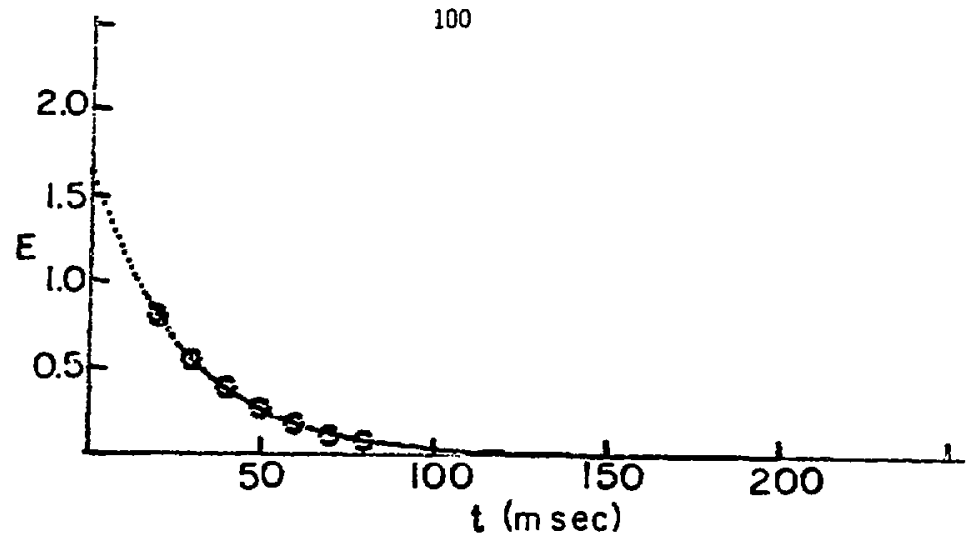

Fig. 22a. Enhancement vs delay time for $0.05 \mathrm{M} \mathrm{Cr}(\mathrm{CN})_{6}^{3-}$. The dotted 7 ine is a fit to $E=E_{0} e^{-\left(t_{d} / T \ln \right)}$ with $\mathrm{T}_{1 n}=27.1 \pm 0.5 \mathrm{msec}$.

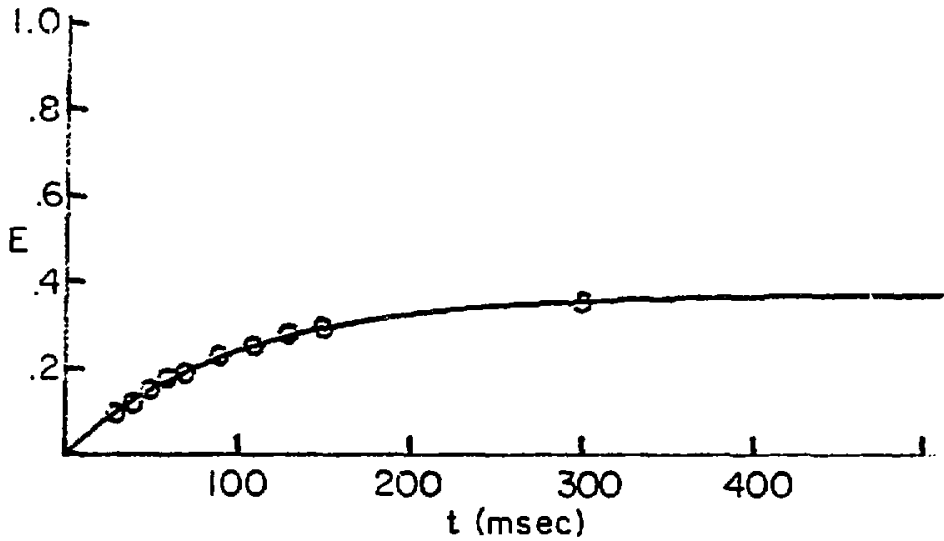

XBL $B 27-5985$

Fig. 22b. Enhancement vs microwave pulse length for methyl protons of $\mathrm{CH}_{3} \mathrm{OD}$ doped with Cu2t. The dotted line is a fit of the form $E=E_{\infty}\left(1-e^{-\left(t_{m} / T \ln \right)}\right)$. 
waveguide one guide wavelength long, and $v_{s}$ is the volume of the sample. The filling factor., is defined

$$
\eta=-\frac{\int_{\text {sample }} H_{1}^{2} d V}{\int_{\text {cavity }} H_{1}^{2} d V}
$$

For this cavity and sample geometry

$$
n=\frac{12.33}{1+(0.82 a / d)^{2}} \frac{V_{s}}{V_{c}},
$$

where $a$ is the cavity radius ard $d$ is its length. $v_{c}$ is the volume of the cavity. $Q_{L}$ for a sample at room temperature is approximately 800. $V_{\omega}$ is $10.6 \mathrm{~cm}^{3}$ at $9.1 \mathrm{GHz}$. The effective volume of the sample is that portion which the coil would enclose. Since the length of the coil is $0.5 \mathrm{~cm}, v_{\mathrm{s}}=8.8 \times 10^{-3} \mathrm{~cm}^{3}$. Insertion of these values and the cavity dimension into Eqs. (6.2) and (5.3) yields $\left\langle\mathrm{H}_{1}^{2}\right\rangle_{\mathrm{s}}=38 \mathrm{G}^{2}$ for $14 \mathrm{~W}$ of incident power wnich is critically coupled to the cavity.

Figure 23 shows an enhancement spectrum of a $<9 \times 10^{-3} \mathrm{M}$ solution of peroxylamine disulphonate dianion in 10 percent $\mathrm{Na}_{2} \mathrm{CO}_{3}$ solution which was taken with $14 \mathrm{~W}$ of power. Peroxylamine disulphonate gives rise to a well known EPR triplet with relativey narrow linewidths. The peak to peak derivative linewidth of this solution was measured to be $0.5 \mathrm{G}$ on a Varian $Y 4502 \times$-Band EPR spectrometer. The width of the 


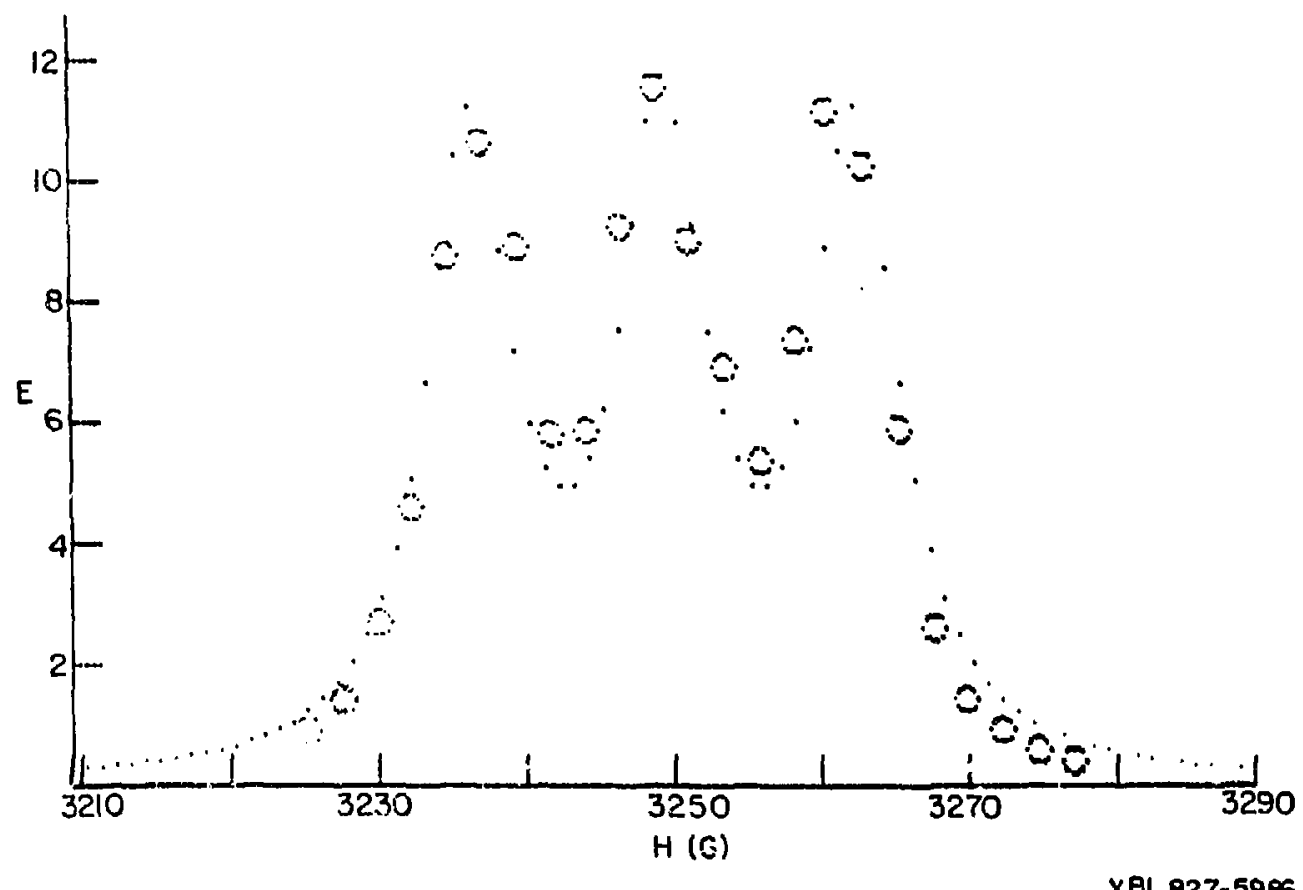

Fig. 23. The enhancement spectrum of peroxylamine disulphonate dicinion taken with $14 \mathrm{~W}$ of microwave power. The dotted line is a simulation according to Eq. (5.4) with $\gamma^{2} H_{1}^{2} T_{1} T_{2}=65$ 
lines in the enhancement spectrum are considerably larger because of the high degree of saturation. According to Eq. (3.32)

$$
E=-\frac{\sigma_{0}}{\rho S_{0}} \sum_{I=-1}^{1} \frac{\gamma_{e}^{2} H_{1}^{2} T_{1 e}{ }^{\top} 2 e}{1+\left(\omega-\omega_{I}\right) T_{2 e}^{2}+\gamma_{e}^{2} H_{1}^{2} T_{1 e} T_{2 e}}
$$

Using the value of $T_{2 e}$ from the low power EPR spectrum the data can be fitted 20 a reasonable extent with $\gamma_{e}^{2} H_{1}^{2} T_{1 e} T_{2 e}=65$. If it is assumed $T_{1 e}=T_{2 e}$ an upper bound for $\left\langle H_{1}^{2}\right\rangle_{s}$ can be calculated to be $12.4 \mathrm{G}^{2}$. This compares to $\left\langle\mathrm{H}_{1}^{2}\right\rangle_{\mathrm{s}}=0.16 \mathrm{G}^{2}$ for $6 \mathrm{~W}$ of incident power for an $X-b$ and DNP spectrometer described by Kramer, Mueller-Warmouth and Schindler (1965), which had the MR coil iriside of the cavity. The agreement between the calculated and measured value of $\left\langle\mathrm{H}_{1}^{2}\right\rangle_{s}$ is about as good as can be expected considering that the theory negiected the effects of the sample on the field and the fact that the experiment was not done under conditions of a perfect match of the cavity to the waveguide.

If we accept the measured estimate of $\left\langle\mathrm{H}_{1}^{2}\right\rangle_{\mathrm{s}}=12.4 \mathrm{G}$, and 0.01 as the smallest enhancement which can be reliably measured, then it is possible to calculate the minimum possible $T_{1 e}$ which can be detectied with this apparatus. Using Eq. (3.33) and assurning $T_{1 e}=T_{2 e}$ and $\sigma / p=1 / 2$, we find that the minimum vetectable $T_{\text {le }}$ would be $9 x$ $10^{-11}$ sec, In practice this value should be increased by a factor of two, because with standard experimental values of $t_{\mathrm{m}}=70 \mathrm{~ms}$, $t_{d}=20 \mathrm{~ms}$ and $T_{1 n}=45 \mathrm{~ms}, \mathrm{Eq}$. (6.1) gives $E_{\text {(observed) }} \approx E / 2$. 
VII. Moving Sample Tube Results

\section{A. Hexacyano-Chromium (III) Ion}

\section{Introduction}

Hexacyano-chromium (III) ion, $\operatorname{Cr}(\mathrm{CN})_{6}^{3-}$, is one of the many cubic complexes of $\mathrm{Cr}^{3^{+}}$which has an easily observable EPR spectrum at room temperature in aqueous solution. Of these complexes, $\mathrm{Cr}\left(\mathrm{H}_{2} \mathrm{O}\right)_{6}^{3+}$ and $\mathrm{Cr}(\mathrm{CN})_{6}^{3-}$ have received the most experimental attention.

Avvarakumov et al. (1960) observed the ERR spectrum of $\mathrm{Cr}\left(\mathrm{H}_{2} \mathrm{O}\right)_{6}^{3+}$ as a function of temperature. They found a single 1 ine of $220 \mathrm{G}$ at room temperature. Similar results were found by Hayes (1961). Botin workers found that the 1 inewidth decreased with increasing temperature. Hayes also measured the linewidth of $\mathrm{Cr}(\mathrm{CN})_{6}^{3-}$ as a function of temperature at both $X$-band $(9 \mathrm{GHz})$ and $\mathrm{S}$-band $(3 \mathrm{GHz})$. The room temperature width of the $\mathrm{Cr}(\mathrm{CN})_{6}^{3-} 1$ ine was $25 \mathrm{G}$ at $\mathrm{x}$-band. Since $\mathrm{ONP}$ enhancements are proportional to the $\mathrm{T}_{1} \mathrm{~T}_{2 e}$ product, the entancement from $\mathrm{Cr}(\mathrm{CN})_{6}^{3-}$ would be nearly two orders of magnitude larger than that from $\mathrm{Cr}\left(\mathrm{H}_{2} \mathrm{O}\right)_{6}^{3+}$ if the relative $T_{1 e}$ of the two ions were similar to the relative $T_{2 e}$. Indeed, the enhancement expected from a line the width of the $\mathrm{Cr}\left(\mathrm{H}_{2} \mathrm{O}\right)_{6}^{3+}$ line begins to approach the limits of detectability with the present DNP apparatus. Consequently DNP measurements were made only on $\mathrm{Cr}(\mathrm{CN})_{6}^{3-}$.

The electronic spin relaxation for $\mathrm{Cr}(\mathrm{CN})_{6}^{3-}$ and all of the cubic $\mathrm{Cr}^{3+}$ complexes has generally been attributed to fluctuations in the quadratic zero-field splitting. For $S=3 / 2$ ions such as these, this mechanism yields the results of Eqs. (3.13)-(3.16) for the 
appropriate relaxation times. In the 1 imit $\omega^{2} \tau_{C}^{2} \ll 1$ these equations lead to a single lorentzian line with a single $T_{1 e}$. When $\omega^{2} \tau_{c}^{2} \gg 1$, Eq. (2.13) predicts that one of the transitions will broaden away. In th is $1 \mathrm{imit}$ the apparent intensity should reduce to $2 / 5$ of its value when $\omega^{2} \tau_{c}^{2} \ll 1$, as the broadened 1 ine disappears into the noise. Experimenta? work on both $\mathrm{Cr}(\mathrm{CN})_{6}^{3-}$ and $\mathrm{Cr}\left(\mathrm{H}_{2} \mathrm{O}\right)_{6}^{3+}$ shows similarity in their relaxation behavior which is consistent with the above mechanism. Hayes found that the linewidths of $\mathrm{Cr}(\mathrm{CN})_{6}^{3-}$ did not change significantly on going from S-band to $x$-band. He also found that over the temperature $r$ ange of $7-60^{\circ} \mathrm{C}$ the decrease in linewidth with temperature could be $\mathrm{fit}$ with an activation energy of $2.6 \mathrm{kcal} / \mathrm{mole}$. The S-b and linewidth of $\mathrm{Cr}\left(\mathrm{H}_{2} \mathrm{O}\right)_{6}^{3^{+}}, 188 \mathrm{G}$ at $26^{\circ} \mathrm{C}$ ( $\mathrm{MCCa}$ in, 1966) is in only moderate agreement with the room temperature $x-b$ and measurements of Kozyrev (1960) and Hayes (1961), al though this difference may be due to different anions used by the various workers. McCain used $\mathrm{Cr}\left(\mathrm{CiO}_{4}\right)_{3}$. The room temperature $X$-band width of $\mathrm{Cr}\left(\mathrm{ClO}_{4}\right)_{3}$ solutions measured by Levonon, Charbinsky and Luz (1970) was $160 \mathrm{G}$. These workers also found that the decrease of the linewidth with temperature for $\mathrm{Cr}\left(\mathrm{H}_{2} \mathrm{O}\right)_{6}^{3+}$ was consistent with an activation energy of about $2 \mathrm{kcal} / \mathrm{mole}$. The small change in the linewidths of both ions on going from S-band to $X-b$ and indicates $\tau_{c}<10^{-11} \mathrm{sec}$. This is consistent with a mechanism involving fluctuations in the second coordination sphere which would affect the instantaneous zero-field splitting.

Levanon, Charbinsky and Luz extended linewith measurements on $\mathrm{Cr}^{3+}$ complexes to $\mathrm{Q}-\mathrm{b}$ and $(35 \mathrm{GHz})$. The linewidth of $\mathrm{Cr}\left(\mathrm{H}_{2} \mathrm{O}\right)_{6}^{3+}$ changed 
from $160 \mathrm{G}$ at $X-b$ and to $72 \mathrm{G}$ at $Q$ band. This allowed them to use Eqs. (2.13) and (2.14) to estimate $\Delta$ and $\tau_{c}$. They found $\tau_{c}=4.9 \times$ $10^{-12} \mathrm{sec}$ and $\Delta=0.087 \mathrm{~cm}^{-1}$. This value of $\Delta$ is of the same order of values of $\Delta$ found in solid complexes of $\mathrm{Cr}^{3+}$ (MeGarvey, 1966). They also added glycerol to solutions of $\mathrm{Cr}\left(\mathrm{H}_{2} \mathrm{O}\right)_{6}^{2+}$ with the intent of increasing viscosities and correlation times. They found that the apparent intensity decreased with increasing mole fraction of glycerol until it reached a liniting value of $2 / 5$ of its original intensity. This is as predicted by Eqs. (2.13) and (2.14). Similar measurements on $\mathrm{Cr}(\mathrm{CN})_{6}^{3-}$ proved somewhat different. On going from $x$-band to $Q-b$ and the linewidth went from 236 to $25 \mathrm{G}$, which is essentially no change. This implies $\tau_{c}<1 \times 10^{-12} \mathrm{sec}$. They felt the relaxation of $\mathrm{Cr}(\mathrm{CN})_{6}^{3-}$ must be controlled by weaker and more rapid fluctuations than are operative in $\mathrm{Cr}\left(\mathrm{H}_{2} \mathrm{O}\right)_{6}^{3+}$.

If $\tau_{c}<1 \times 10^{-12} \mathrm{sec}$ then surely $T_{1 e}=T_{2 e}$ at $x$-band for $\mathrm{Cr}(\mathrm{CN})_{6}^{3-}$. For this reason $\mathrm{Cr}(\mathrm{CN})_{6}^{3-}$ ion is attractive as a $T_{1 e}$ standard. The abundant isotope of chromium, ${ }^{52} \mathrm{Cr}$, has zero nuclear sp in and 90.5 percent natural abundance. ${ }^{53} \mathrm{Cr}(I=3 / 2)$ is only 9.5 abundant. The hyperfine structure from ${ }^{53} \mathrm{Cr}$ is not resolved in $\mathrm{Cr}(\mathrm{CN}){ }_{6}^{3-}$. Its contribution to the DNP can be neglected for most purposes. Hence, in $\mathrm{Cr}(\mathrm{CN})_{6}^{3-}$ there exists a possible DNP standard which is not complicated by fine-st)weture or hyperfine structure. While $T_{10}$ for $\operatorname{Cr}(\mathrm{CN})_{6}^{3-}$ cannot be calculated from measured spin Hamiltonian parameters in the sense that the $\mathrm{VO}^{2+} \mathrm{T}_{1 e}$ can, $\mathrm{T}_{2 e}$ for $\operatorname{Cr}(\mathrm{CN})_{6}^{3-}$ is availaole from an EPR linewidth measurement. It is likely that $T_{1 e}$ can be determined for 
$\mathrm{Cr}$ (CN) ${ }_{6}^{3-}$ more accurately from linewidth measurements than $\mathrm{T}_{1 \mathrm{e}}$ can be calculated from theory for $\mathrm{VO}^{2+}$. ONP measurements on $\mathrm{Cr}(\mathrm{CN})_{6}^{3-}$ should serve as a test of the cansistency of the method.

\section{Experimental}

Solutions containing $\mathrm{Cr}(\mathrm{CN})_{6}^{3-}$ were made by dissolving weighed amounts of $\mathrm{K}_{3} \mathrm{Cr}(\mathrm{CN})_{6}$ (Pfaltz and Bauer) in distilled water. DNP enhancements were measured in the moving sample tube spectrometer described earlier. At each temperature the enhancement of a $y^{2+}$ standard was also measured. Initially $\mathrm{VOSO}_{4}$ solutions were used. Later enhancement measurements of $\mathrm{VOSO}_{4}$ solutions vs $\mathrm{VO}\left(\mathrm{ClO}_{4}\right)_{2}$ solutions showed significant differences between perchlorate and sulfate solutions. Enhancements were renormalized to allow comparison of $\mathrm{Cr}(\mathrm{CN})_{6}^{3-}$ data with $\mathrm{vO}\left(\mathrm{ClO}_{4}\right)_{2}$ data.

Solutions for mixed solvent work were made by first preparing a solution of the paramagnetic ion in $\mathrm{H}_{2} \mathrm{O}$ or $\mathrm{D}_{2} \mathrm{O}$, then diluting with the other solvent to the desired volume. Reagent grade di-methyl-sulfoxide (OMSO), acetonitrile, and methanol were used in mixed solvent studies. Linewidths for the mixed solvent studies were measured with a Varian $V-4502$ EPR spectrometer on samples in standard capillary tubes.

3. Results and Discussion

$T_{\text {le }}$ values for $\mathrm{Cr}(\mathrm{CN})_{6}^{3-}$ were calculated from meassured erihancements according to an equation similar to Eq. (5.17), with the proper correction for the absence of hyperfine lines. linewidths for $\operatorname{Cr}(\mathrm{CN})_{6}^{3-}$ were taken from measurements by Hayes (1961). 
The $\sigma / p$ corrections prove interesting for this ion. If no $\sigma / p$ correction is made, the resulting values of $\mathrm{T}_{1 \mathrm{e}}$ are plotted in Fig. 24. It is found that generally $T_{1 e} \neq T_{2 e}$ and that the temperature dependence of $T_{1 e}$ is somewhat different from $T_{2 e^{*}}$. This is contrary to the expectations of theory. Clearly a a/p correction must be necessary. However, assumption of a rotational modulation of the dipolar interaction to make the o/p correction for $\operatorname{Cr}(\mathrm{CN})_{6}^{3-}$ would require an unreasonably short rotational correlation time for $\mathrm{Cr}(\mathrm{CN})_{6}^{3-}$ in order to make the data approach theoretical expectations.

Guenther and Pfeifer (1964) found that the frequency dependence of proton spin-lattice relaxation in aqueous solutions of $\mathrm{Cr}(\mathrm{CN})_{6}^{3-}$ could be best fit by translational modulation of the dipolar interaction. Using the best $f$ it value of $\tau_{d}$ from their measurements, $\tau_{d}\left(21^{\circ} \mathrm{C}\right)=$ $3.3 \times 10^{-11} \mathrm{sec}$, and Eqs. (3.16) and (3.17), a proper o/p correction leads to the $T_{l e}$ values plotted in Fig. 25 . With this correction, the experimentally measured $T_{1 e}$ 's and the theoretical expectation that $T_{1 e}=T_{2 e}$ are in reasonable agreement.

The idea that the interaction between the electrons of $\mathrm{Cr}(\mathrm{CN})_{6}^{3-}$ and the solvent protons is governed by translational motion is not too surprising. Since the paramagnetic center is completely surrounded by $\mathrm{CN}^{-}$ligands, the only interactions possible between the solvent and paramagnetic ion are through the second and higher coordination spheres. It is reasonable to expect that the lifetime of a proton in the second coordination sphere of the chromium ion is less than the correlation time for the second sphere complex to tumble as a whole, 


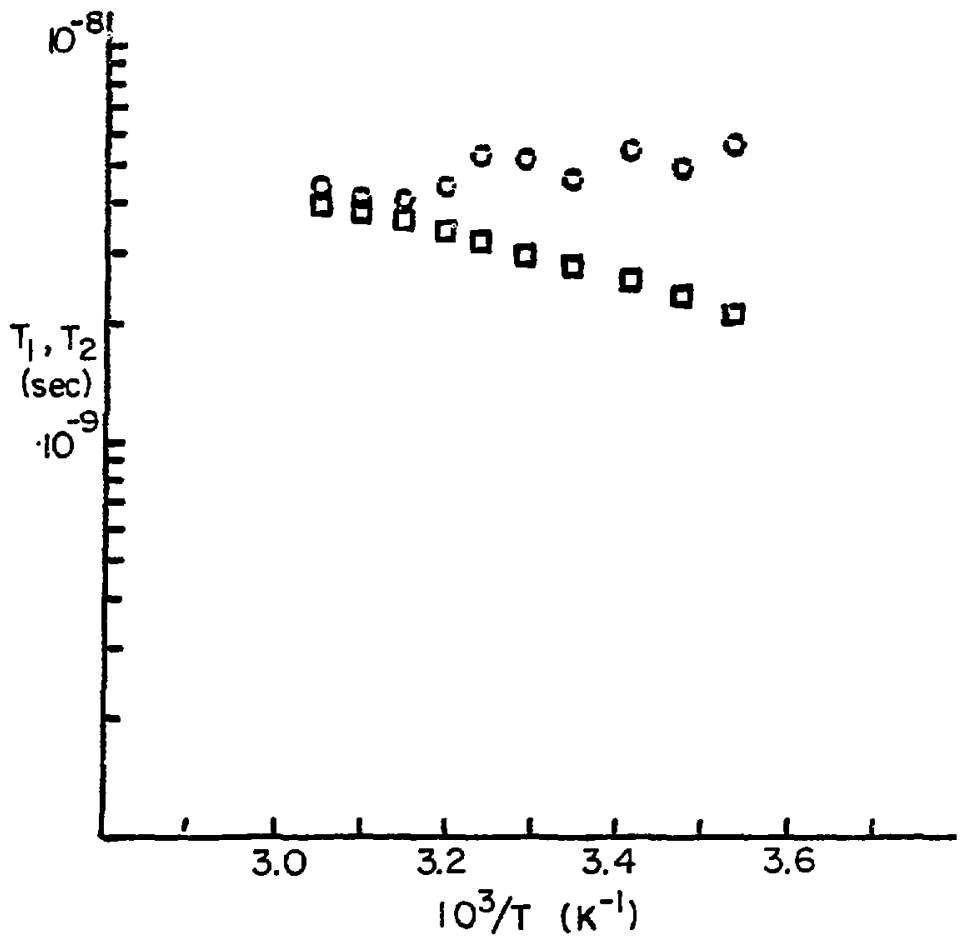

XBL $827-5987$

Fig. 24. $T_{1}$ and $T_{2}$ for $\operatorname{Cr}(C N)_{6}^{3-}$. Circles-T $T_{1}$ from DNP with no $\sigma / \rho$ correction. Squares-T2 from 1 inewidth measurements (Hayes, 1961). 
especially since there is no hydrogen bonding to the first sphere ligands.

The experiments on mixed solvent solutions were initially done merely to demonstrate that DNP could be done with this apparatus on systems with more than one proton resonance. These data by no means represent a complete study, but do yield some interesting qualitative information.

ONP measurements were compared between solutions of $0.055 \mathrm{~m}$ $\mathrm{Cr}(\mathrm{CN})_{6}^{3-}$ in $2: 1$ by volume mixtures of acetonitri le and $\mathrm{H}_{2} \mathrm{O}$, and $1: 1$ mixtures of acetonitrile and $\mathrm{D}_{2} \mathrm{O}$. The measurements vere made at room temperature. The proton signals from water and acetonitrile were not completely resolved, so enhancement information from this solution was obtained from curvefitting. The curvefitting process was somewhat time consuming, so only a few spectra were averaged, resulting in poor precision. The problem was particutarly severe for the acetonitrile protons because the signal from then was less intense than the $\mathrm{H}_{2} \mathrm{O}$ protons due to the smaller concentration of $\mathrm{CH}_{3} \mathrm{CN}$. The solution. containing $\mathrm{D}_{2} \mathrm{O}$ was examined to obtain more precise information on the acetonitrile protons. The resulting proton $T_{1}$ values were $T_{1}\left(\mathrm{CH}_{3} \mathrm{CN}\right)=25 \pm 3 \mathrm{msec}, T_{1}\left(\mathrm{H}_{2} \mathrm{O}\right)=33 \pm 8 \mathrm{msec}$. The enhancements were $E_{\mathrm{CH}_{3} \mathrm{CN}}=1.3: 0.2, E_{\mathrm{H}_{2} \mathrm{O}}=1.3 \pm 0.5$. The data quoted for $\mathrm{CH}_{3} \mathrm{CN}$ were obtained from the mixed solution with $\mathrm{O}_{2} \mathrm{O}$. The degree of interaction with the $\operatorname{Cr}(\mathrm{CN})_{6}^{3-}$ ion seems to be roughiy the same for both the acetonitrile and water protons. This is consistent with the interaction being a second sphere process, with roughly equal participation of 
$\mathrm{CH}_{3} \mathrm{CN}$ and $\mathrm{H}_{2} \mathrm{O}$ in the second coordination sphere. (Strictiy speaking, the measured $T_{1}$ and enhancement of the acetonitrile protons should be smaller than the $\mathrm{H}_{2} \mathrm{O}$ measurements because of the slight ly increased viscosity of the $\mathrm{D}_{2} \mathrm{O}$ mixture. The precision of the measurements was not gre at enough to discern this aifference.) Measurements on $0.02 \mathrm{M}$ $\mathrm{vO}^{2+}$ in a $1: 1$ by volume mixture of acetonitrile and $\mathrm{H}_{2} \mathrm{O}$ yielded strikingly different results. It was found that $T_{1}\left(\mathrm{CH}_{3} \mathrm{CN}\right)=730 \mathrm{msec}$ and $T_{1}\left(\mathrm{H}_{2} \mathrm{O}\right)=45 \mathrm{msec}$. This is clearly illustrative of the difference between a second sphere interaction and a first sphere interaction. $y^{2+}$ apparently preferentialiy forms complexes with $\mathrm{H}_{2} \mathrm{O}$. Because of the long $T_{1 n}$ of the acetonitrile protons and the need to keep microwave pulse lengths to -100 msec to avoid heating the sample excessively, accurate extrapolation of enhancement data to infinite pulse lengths was difficult. The extrapolated values were $\mathrm{E}_{\mathrm{CH}_{3} \mathrm{CN}}=3.1 \pm 1.7$ and $E_{\mathrm{H}_{2} \mathrm{O}}=1.2 \neq 0.1$. Since $E_{\mathrm{H}_{2} \mathrm{O}}$ is determined by rotational modulation and $\mathrm{E}_{\mathrm{CH}_{3} \mathrm{CN}}$ is presumably determined by translation, it is reasonable to expect the enhancements to differ, although a quantitative comparison is not possible because of the large uncertainty in the data. Qualitatively, because of the difference in $0 / \rho$, translational modulation would be expected to produce larger enhancements than rotational modulation at room temperature.

The results of EPR linewidth measurements on $\mathrm{Cr}(\mathrm{CN})_{6}^{3-}$ ion in mixed solvent solutions are shown in Table III. All of the mixed solvents show increased 1 inewidths over the pure $\mathrm{H}_{2} \mathrm{O}$ system. This is certainly not a viscosity effect, at least in a macroscopic sense, since $\mathrm{CH}_{3} \mathrm{CN}$ 
and $\mathrm{CH}_{3} \mathrm{OH}$ are less viscous than water. One possible explanation is that the mixed solvent system has a less symmetric second coordination sphere around $\mathrm{Cr}(\mathrm{CN})_{6}^{3-}$. This would result in a larger instantaneous zerofield splitting and more efficient relaxation. It is difficult to say what the effect of a mixed solvent system would be on the correlation time. These measurements at least support the assertion that fluccuations in the second coordination sphere are important for electron spin relaxation in $\mathrm{Cr}(\mathrm{CN})_{6}^{3-}$.

The DNP measurements on $\mathrm{Cr}(\mathrm{CN})_{6}^{3-}$ in pure water indicate that it is reasonable to assume $T_{1 e}=T_{2 e}$ at $x-b$ and. It should indeed be possible to use $\mathrm{Cr}(\mathrm{CN})_{6}^{3-}$ as a standard for $\mathrm{T}_{1 \mathrm{e}}$ measurements. In some respects it seems that the more interesting experiment would have been to use $\mathrm{Cr}(\mathrm{CN})_{6}^{3-}$ as a standard for measurements of $\mathrm{T}_{\text {le }}$ for each of the eight $\mathrm{vo}^{2+}$ lines as a function of temperature. In any case, these measurements show that the theoretical calculation of $\mathrm{T}_{1 \mathrm{e}}$ for $\mathrm{vo}^{2+}$ from measured spin-Hamiltonian parameters, and the theoretical prediction that $\mathrm{T}_{1 \mathrm{le}}=\mathrm{T}_{2 e}$ for $\mathrm{Cr}(\mathrm{CN})_{6}^{3-}$ at $\mathrm{X}$-band are consisterit. B. $\mathrm{Cu}\left(\mathrm{H}_{2} \mathrm{O}\right)_{6}^{2+}$

1. Introduction

Hexa-aquo copper (II) ion, $\mathrm{Cu}\left(\mathrm{H}_{2} \mathrm{O}\right)_{6}^{2+}$, is a $3 d^{9}$ ion. In an octahedral crystal field the ground state would be the orbitally degenerate ${ }^{2} E_{g}$. The Jahn-Teller theorm (Jahn and Teller, 1937) requires that the complex distort, in order to break this orbital degeneracy. A tetragonal distortion, an elongation along the $z$ axis, yieids an orbitally non-degenerate ${ }^{2}{ }{ }_{1 g}$ ground state. This corresponds to a 
Table III. Room temperature $\left(23^{\circ} \mathrm{C}\right)$ Linewidths of $\mathrm{Cr}(\mathrm{CN})^{3-}$.

\begin{tabular}{cc} 
Mixture & $\Delta \mathrm{H}_{\mathrm{pp}}(\mathrm{G})$ \\
\hline Pure $\mathrm{H}_{2} \mathrm{O}$ & 23 \\
$1: 1$ acetonitrile $/ \mathrm{H}_{2} \mathrm{O}$ & 46 \\
$1: 1$ acetonitrile $/ \mathrm{O}_{2} \mathrm{O}$ & 50 \\
$1: 1 \mathrm{CH}_{3} \mathrm{OH} / \mathrm{H}_{2} \mathrm{O}$ & 52 \\
$1: 1 \mathrm{OMSO}_{2} \mathrm{O}$ & 106 \\
\hline
\end{tabular}


positive hole, and an unpaired electron in a $3 d x^{2}-y^{2}$ orbital. The ${ }^{2} \mathrm{~A}_{1 \mathrm{~g}}$ state, corresponding to the unpaired electron being in the $3 \mathrm{~d}_{z}{ }^{2}$ orbital, would be expected to be a low-lying excited state for small tetragonal distortions. The ${ }^{2} \mathrm{~A}_{1 g}$ state, however, is not connected to the ${ }^{2}{ }_{1 g}$ ground state by spin-orbit coupling. This makes rapid spin1 attice relaxation by the Van Vleck direct or Raman processes unlikely (Kivelson, 1966). Consequently, the EPR spectrum of $\mathrm{Cu}\left(\mathrm{H}_{2} \mathrm{O}\right)_{6}^{2+}$ is observable at room temperature in crystals and in solution.

Early observation of the EPR spectrum of $\mathrm{Cu}\left(\mathrm{H}_{2} \mathrm{O}\right)_{6}^{2+}$ at room temperature by Kozyrev (1955) revealed a single line with a peak-to-peak linewidth of abnut 150 gauss. This was somewhat surprising, since both abundant isotopes of copper, ${ }^{63} \mathrm{Cu}$ and ${ }^{65} \mathrm{Cu}$, have their nuclear spin, $1=3 / 2$. If the hyperf ine coupling constant were 1 arge compared to the linewidth one would expect to see a quartet. Kozyrev initially attributed the lack of hyperfine structure to the formation of aggregations of $\mathrm{Cu}^{2+}$ ions in which the hyperf ine coupling was presumably exchanged away. This assertion was based on observations with rather concentrated solutions. When it became clear that the EPR spectrum was essentially the same in more dilute solutions, where the formation of aggregations was unlikely, this explanation had to be discarded. A more likely explanation was that the hyperfine lines were simply much broader than the separation between them. McGarvey (1957) suggested that the broadening was due to a combination of slow tumbling as predicted by McConnell's (1956) theory and an interaction with a low lying excited state. 
It should be noted that some complexes of $\mathrm{Cu}^{2+}$ do have EPR spectra with resolvable ${ }^{63} \mathrm{Cu}$ hyperf ine structure in aqueous sulution at rooni temperature. The ethylendiamine complex considered earlier in this work is one such example. The $\mathrm{Cu}(\mathrm{en})_{2}\left(\mathrm{H}_{2} \mathrm{O}\right)_{2}^{2+}$ complex not only has narrower lines than the hexaquo complex, but also has a larger ${ }^{63} \mathrm{Cu}$ hyperf ine coupling constant. Hayes (1961) was able to ooserve some hyperfine structure for $\mathrm{Cu}_{(}\left(\mathrm{H}_{2} \mathrm{O}_{6}^{2+}\right.$ near $0^{\circ} \mathrm{C}$. He est imated that the hyperfine coupling constant was between -31 and -38 gauss. A more precise determinat,ion by Chang (1971) using least squares fitting of the spectrum yielded a value of $-34.5 \mathrm{G}$ for the isotropic coupling constant near room temperature. The isotropic ${ }^{63} \mathrm{Cu}$ coupling corstant for $\mathrm{Cu}(\mathrm{en})_{2}\left(\mathrm{H}_{2} \mathrm{O}\right)_{2}^{2+}$ determined by Lewis, Alei and Morgan (1966b) was $-85.5 \mathrm{G}$. This is quite a significant difference. Part of the difference can be explained by an indirect dipolar (pseudo-contact) interaction. This can be shown with a simple calculation. McGarvey (1967) gives a simple expression for the hyperfine coupling constant,

$$
A=P(-\kappa+(g-2.0023))
$$

where $P \propto\left\langle 1 / r^{3}\right\rangle$ for the orbital containing the unpaired electron and $g$ is the isotropic $g$ factor. The parameter $\alpha$ gives rise to the true Fermi-contact interaction and depends on the amount of polarization of $s$ electrons induced by the presence of the unpaired d electron. For transition metal ions $k$ is usually positive (McGarvey, 1966). The tem involving the $g$ factor describes the psuedo-contact interaction. 
A reasonable value for $P$ is about $400 \mathrm{~cm}^{-1}$ (McGarvey, 1967). If values of $A$ and $g$ measured from frozen glasses of $\mathrm{Cu}\left(\mathrm{H}_{2} \mathrm{O}\right)_{6}^{2^{+}}$(Chang, 1971) and $\mathrm{Cu}(\mathrm{en})_{2}\left(\mathrm{H}_{2} \mathrm{O}\right)_{2}^{2+}$ (Lewis, Alci and Morgan, 1966b) are used, and $P$ is assumed to be the same for both complexes, then the values of $k$ calculated for $\mathrm{Cu}\left(\mathrm{H}_{2} \mathrm{O}\right)_{6}^{2+}$ and $\mathrm{Cu}(e)_{2}{ }_{2}\left(\mathrm{H}_{2} \mathrm{O}\right)_{6}^{2+}$ are 0.31 and 0.32 respectively. Thus the pseudo-contact interaction seems to make up most of the difference between the hyperfine ccupling constants. However, the ${ }^{63} \mathrm{Cu}$ hyperfine coupling which was observed for $\mathrm{Cu}\left(\mathrm{H}_{2} \mathrm{O}\right)_{6}^{2+}$ in the glassy state is $-46.9 \mathrm{G}$, which is of considerably larger magnitude than the value observed in solution near room temparature. The hyperfine coupling constant for $\mathrm{Cu}(\mathrm{en})_{2}\left(\mathrm{H}_{2} \mathrm{O}\right)_{2}^{2+}$ observed in the glassy state, $0.0086 \mathrm{~cm}^{-1}$, is not significantly different from the value observed in solution, $0.0084 \mathrm{~cm}^{-1}$. Furthermore, Chang (1971) noticed that the hyperfine constant for $\mathrm{Cu}_{2}\left(\mathrm{H}_{2} \mathrm{O}\right)_{6}^{2+}$ in solution decreased significantly with increasing temperature. Wilson (1975) observed a similar temperature dependence of the ${ }^{63} \mathrm{Cu}$ hyperfine coupling constant in the $\mathrm{Cu}\left(\mathrm{NH}_{3}\right)_{6}^{2+}$ complex in liquid ammonia. He attrituted the effect to a temperature dependent mixing of the ground state with the ${ }^{A}{ }_{1 g}$ excited state. He felt that the hyperfine coupling constant in the excited state must be smeller than in the ground state.

The mixing of the ground and first excited state is possible through a dynamic Jahn-ieller effect. This effect is discussed by 0'Brien (1964). A vibrational mode can mix the ground state and the ${ }^{2}{ }_{A_{1 g}}$ excited state. In a classical sense the degree of mixing depends on the vibrational amplitude. Quantum mechanically, the electronic 
and vibrational motion are not completely separable. An increase in temperature cause the occupation of higher vibronic states which contain a greater admixture of the excited electronic state.

The ${ }^{63} \mathrm{Cu}$ hyperfine coupling constant in the ${ }^{2} \mathrm{~A}_{1 \mathrm{~g}}$ state is expected to be of smaller magnitude than the in ground state because in tetragonal symmetry the $A_{1 g}$ state has the same symmetry as the copper $s$ orbitals, and can mix with them. Thus the ${ }^{2} \mathrm{~A} g \mathrm{~g}$ state has some $s$ character. This $s$ character results in a direct Fermi-contact interaction of the opposite sign of that induced by spin-polarization, and thus lowers the magnitude of the hyperfine constant. Mixing of the ${ }^{2} \mathrm{~A}$ state with the ground state results in a lowered magnitude of the observed hyperf ine constant in solution.

The lack of resolution of the hyperfine structure forced many workers (Avvakumov, et al. 1959, Fujiwara and Hayashi, 1965) to use the peak-to-peak derivative width of the single line observed for $\mathrm{Cu}\left(\mathrm{H}_{2} \mathrm{O}\right)_{6}^{2+}$ as a measure of $\mathrm{T}_{2 e^{*}}$. Because the contribution of the unresolved hyperfine structure to the 1 inewidth is not negligible over a large temperature range this method cannot give quantitative results. These meastrements, however, produced two import ant qualitative cbservations for $\mathrm{Cu}\left(\mathrm{H}_{2} \mathrm{O}_{6}^{2^{+}}\right.$. First, above room temperature, the linewidth clearly increased with temperature. Secondly, at low temperatures where some hyperf ine structure was resolvable, it was apparent that the linewidth depended on $M_{I}$. This suggested that a McConne 11 (1956) tumbling mechanism was important at low temperatures. Since 
McConnell's theory predicts a linewidth which decreases with increasing temperature, the increased 1 inewidth at higher temperatures must be ciue to a different mechanism.

Spencer (1965) studied the EPR spectrum of $\mathrm{Cu}\left(\mathrm{H}_{2} \mathrm{O}_{6}^{2+}\right.$ as a function of temperature at $x$-band. He felt that some of the $M_{I}$ dependent, low temperature linewidth could be ascribed to the McConnell (1956) tumbling mechanism. However, he also realized that the $\mathrm{Cu}\left(\mathrm{H}_{2} \mathrm{O}\right)_{6}^{2+}$ complex should be capable of undergoing a pseudo-rotation in which the major symmetry axis of the complex changes with respect to a spacefixed coordinate system. This psuedo-rotation, or inversion as it is sometimes called, $c$ an also cause $M_{I}$ dependent relaxation. Spencer developed a simple theory to treat relaxation by the inversion mechanism, but his theory treated the tumbling process and inversion separately, which could not be correct. Later work seemed to conf irm that tumbling alone could not account for the low temperature $M_{I}$ dependent linewidth. Lewis, Alei and Morgan (1966a) used computer simulations to extract the individual hyperf ine linewidths for $\mathrm{Cu}\left(\mathrm{H}_{2} \mathrm{O}\right)_{6}^{2+}$. They found that an application of Kivelson's (1960) tumbling theory to the $M_{1}$ dependence of the $l$ inewidths required a room temperature rotational correlation time of $3 \times 10^{-12} \mathrm{sec}$. This is much too short for an ion the size of $\mathrm{Cu}\left(\mathrm{H}_{2} \mathrm{O}\right)_{6}^{2+}$. Chang (1971) used a least-squares program to determine the individual hyperf ine linewidths and he came to essentially the same conciusion. The hydrodynamic radius necessary to account for the $M_{I}$ dependent 1 inewidth, $1.71 \AA$ is much too small for $\mathrm{Cu}\left(\mathrm{H}_{2} \mathrm{O}\right)_{6}^{2+}$. 
Noack, Kokoszka and Gordon (1971) noticed a similar anomalously low tumbling correlation time for the symmetrically substituted conplex of 2, 2' dipyridine, Cu(dipy) $3_{3}^{2+}$. They explained this result by concluding that symmetric complexes could undergo rapid inversion associated with a dynamic Jahn-Teller effect. They felt that the apparent correlation time, $\mathrm{T}$, was given by

$$
\frac{1}{T_{C}}=\frac{1}{T_{R}}+\frac{1}{T_{J}-T}
$$

where $\tau_{R}$ is the rotational correlation time and $\tau_{J-T}$ was the correlation time for inversion. A more complete treatment by Rubinstein, Barum and Luz (197I), showed that inversion and tumbling can be treated together with the apparent correlation time $\tau_{c}$, given by

$$
\frac{1}{\tau_{C}}=\frac{1}{\tau_{R}}+\frac{n}{n-1} \frac{1}{\tau_{j}}
$$

where $\tau_{i}$ is the correlation time for the inversion process and $n$ is the number of equivalent axis a long which distortion can occur. In this case $n$ is 3 . Poupko and Luz (1971) measured linewidths for $\mathrm{Cu}\left(\mathrm{H}_{2} \mathrm{O}\right)_{2}^{2+}$ at $X$-band and $Q$ band. They found $\tau_{i}\left(25^{\circ} ;=1.2 \times 10^{-11}\right.$ sec.

Lewis, Alei and Morgan (1966a) did not interpret their data on the bas is of the inversion mechanism. They felt that the apparent anomalously short correlation time was caused by the anisotropic parameters 
$\Delta g=g_{\|}-g_{\perp}$ and $\Delta A=A_{\|}-A_{\perp}$ being smaller in solution than what was measured in the glassy state. They (Lewis and Morgan, 1968) based this assertion on the observation that $\Delta g$ is reduced in some crystal1 ine salts containing $\mathrm{Cu}\left(\mathrm{H}_{2} \mathrm{O}\right)_{6}^{2+}$ as the temperature is raised (Baggaley and Griffiths, 1952). Linewinth measurements on $\mathrm{Cu}\left(\mathrm{NH}_{3}\right)_{6}^{2+}$ ion in liquid armonia by Schmidt and Breitschwerdt (1974) support this interpretation. They were able to estimate the product $\Delta A \Delta g$ from the dependence of the $B$ parameter of Eq. (2.3) on viscosity divided by temperature. They estimated a value of $\Delta g \Delta A$ which was 55 percent of the value determined from measurements on the $\mathrm{Cu}\left(\mathrm{NH}_{3}\right)_{6}^{2+}$ system in the glassy state.

Fast inversian as an explanation of the apparent short correlation time seems more attractive to us than a reduction in $\Delta g$ and $\Delta A$ from the ir values in a glass. The apparent reduction of $\Delta g$ observed in solid is probably due to a dynamic Jahn-Teller inversion which causes a reduction in $\Delta g$ in the same sense that the NMR $l$ ines from a nucleus exchanging between inequivalent positions appear to move together as the exchange rate increases. If inversion were fast enough, the spectrum would be isotropic, and the linewidths would be expected to depend on the magnitudes of $\Delta g$ and $\Delta A$ which existed when the motion was frozen (0'brien, 1964). Surely the applicable values of $\Delta g$ and $\Delta A$ in solution should also be those in which the motion is frozen as completely as possible. A measurement comparing the contribution of this $M_{I}$ dependent mechanism to $T_{1 e}$ with $i$ ts contribution to $T_{2 e}$ when $\omega_{s} \tau_{c} \geq 1$ would provide an unambiguous determination of the correlation 
time for the mechanism. Unfortunately this measurement proved impossible for $\mathrm{Cu}\left(\mathrm{H}_{2} \mathrm{O}\right)_{6}^{2+}$ for reasons which are discussed later. However, such a measurement might be possible for $\mathrm{Cu}\left(\mathrm{NH}_{3}\right)_{6}^{2^{+}}$, which has a better resolved spectrum and can be observed at temperatures where the $M_{I}$ dependent contribution to $\mathrm{T}_{1 \mathrm{e}}$ is more significant.

The linewidth of $\mathrm{Cu}\left(\mathrm{H}_{2} \mathrm{O}_{6}^{2+}\right.$ above room temperature is dominated by spin-rotation. The importance of spin-rotation as a relaxation mechanism for copper complexes was demonstrated by Wilson and Kivelson (1966b) with their work on copper acetylacetonate in organic solvents. For $\mathrm{Cu}\left(\mathrm{H}_{2} \mathrm{O}\right)_{6}^{2+}$, with $\mathrm{g}_{\|}=2.387$ and $\mathrm{g}_{1}=2.072$ (Chang, 1971), Eq. (2.8) predicts a linewidth contribution of $57 \mathrm{G}$ for $\tau_{R}=2 \times 10^{-11} \mathrm{sec}$. This is close to the observed linewidth at room temperature after the contribution from the low temperature tumbiing or inversion mechanism is substracted. However, Lewis Alei and Morgan (1966a) noticed that the temperature dependence of the high temperature 1 inewidth was somewhat less than that of $T / \eta$, which would be predicted for pure spin-rotation. While Chang (1971) could draw a reasonable straight line through his plot of the $M_{I}$ independent linewidth for $\mathrm{Cu}\left(\mathrm{H}_{2} \mathrm{O}\right)_{6}^{2+}$ vs $\mathrm{T} / \mathrm{n}$, the intercept at $T / n=0$ indicated a residual linewidth of about $30 \mathrm{G}$. This is strongly suggestive that an additional relaxation mechanism besides spin-rotation and tumbling is important for $\mathrm{Cu}\left(\mathrm{H}_{2} \mathrm{O}\right)_{6}^{2+}$. While Eq. (a.8) is the result of perturbation theory and is thus only approximate, the residual linewidth seems much too large to be blamed on uncertainty in the theory. 
On the basis of order of magnitude calculations, Kivelson (1965) suggested that the residual width might be accounted for by an Orbach process. Kivelson's estimate assumed that the energy separation between the $B_{1 g}$ ground $s$ tate and the $A_{1 g}$ excited state be the order of $1000 \mathrm{~cm}^{-1}$. Wilson (1975) reconsidered Chang's (1971) data. After subtracting the effect of tumbling and spin-rotation from the observed linewidth he found that the activation energy of the remaining width was only a few hundred waverumbers. He felt that this excluded the Orbach process, since the lowest excited state should be more than a few hundred wavenumbers above the ground state. Wilson proposed that the residual width could be a solution Raman process, in spite of Kivelson's (1956) calculation that the Raman process should be less important than the Orbach process for relaxation in $\mathrm{Cu}\left(\mathrm{H}_{2} \mathrm{O}\right)_{6}^{2+}$. If the energy separation between the ground and first excited electronic state, $\delta_{o n}$, were significantly larger than the $1000 \mathrm{~cm}^{-1}$ estimated by Kiveison, the Raman process would be more important. However, the magnitude of the Raman contribution is independent of $\delta_{\text {on }}$. Kivelson estimated that the Raman process would only contribute $-5 G$ to the linewidth of $\mathrm{Cu}\left(\mathrm{H}_{2} \mathrm{O}\right)_{6}^{2+}$. Wilson found that if he used a hydrodynamic radius of $3.47 \AA$ to calculate the spin-rotational width, the remaining width after spin rotation and tumbling were substracted varied as $T^{1 / 2}$, which is expected for the Raman process.

Valiev and Zaripov (1966) developed a theory describing relaxation in $\mathrm{Cu}\left(\mathrm{H}_{2} \mathrm{O}\right)_{6}^{2+}$. They felt that the primary cause of broadening of the EPR signal was transitions between the ground electranic state and the 
lowest excited electronic state. These transitions proceeded without spin flipping. They were caused by vibrational modulation of the crystal field in the manner discussed by Altschuler and Valiev (1959). Valiev and Zaripov found that

$$
1 / T_{2} \approx 1 / \tau_{a}
$$

where $\tau_{\mathrm{a}}$ was the lifetime of the molecule in the ground electronic state. Their calculations led to $\tau_{a}^{-1} \approx 10^{9} \mathrm{sec}^{-1}$. These calculations cannot be correct, since they account for virtually all of the linewidth of $\mathrm{Cu}\left(\mathrm{H}_{2} \mathrm{O}\right)_{6}^{2+}$ before sp in rotation is even considered. However, they are interesting since they suggest a relaxation mechanism which does not affect $T_{1 \mathrm{e}}$. Perhaps the residual linewidth is not due to a spin-lattice process.

McCain and Myers (1974) made $T_{1 e}$ measurements on $\mathrm{Cu}\left(\mathrm{H}_{2} \mathrm{O}\right)_{6}^{2+}$ using $\mathrm{O}$ NP at S-band. They found that at $5^{\circ} \mathrm{C} T_{l e}=2 T_{2 e}$, but that at $60^{\circ} \mathrm{C} T_{1 e}$ was beginning to approach $T_{2 e}$. The result at $5^{\circ} \mathrm{C}$ cannot be explained by slow tumbling modulation of anisotropic $g$ and $A$ values. At $S$ band $\omega^{2}{ }^{2}{ }_{R}^{2}=0.3$. If it is assumed that the appropriate correlation time for this mechanism is $2 / 3 \tau_{i}$, then $\omega^{2} \tau_{c}^{2}<0.05$. The $T_{1} / T_{2}$ ratio should be near its excreme narrowing value of $7 / 6$ (Poole and Farrach, 1968). Also, spin-rotation should still be more important than tumbling even at this low temperature. For spin rotation the contribution to $T_{1 e}$ and $T_{2 e}$ should be equal. 
The present DNP work on $\mathrm{Cu}\left(\mathrm{H}_{2} \mathrm{O}\right)_{6}^{2+}$ at $\mathrm{X}$-band was undertaken with the hope of resolving some of the confusion concerning electron spin relaxation in $\mathrm{Cu}\left(\mathrm{H}_{2} \mathrm{O}\right)_{6}^{2+}$. Of all of the paramagnetic ions studied in this work, $\mathrm{Cu}\left(\mathrm{H}_{2} \mathrm{O}\right)_{6}^{2+}$ is the most poorly understood and consequently has the greatest potential for providing interesting new infornation. Unfortunately, because of its unresolved hyperfine structure and relatively short relaxation times it is also the most difficult to study.

\section{Experimenta?}

Aqueous solutions of $\mathrm{Cu}^{2+}$ ion were prepared by dissolving weighed amounts of $\mathrm{Cu}\left(\mathrm{ClO}_{4}\right)_{2} \cdot 6 \mathrm{H}_{2} \mathrm{O}$ ( $\mathrm{Alfa}$-Ventron) in distilled water. Solutions were analyzed for $\mathrm{Cu}^{2+}$ by adding an excess of ethylenediamine and measuring the visible absorption at $550 \mathrm{~nm}$. The molar extinction coefficient of $\mathrm{Cu}(e n){ }_{2}\left(\mathrm{H}_{2} \mathrm{O}\right)_{2}^{2+}$ complex was determined by dissolving a weighec amount of copper wire in nitric acid, adding an excess of ethylendiamine and taking a visible spectrum on a Cary 14 visible spectrometer. The value of the molar extinction coefficient determined in this manner, $\varepsilon=62.5$ was in reasonable agreement with the value, $\varepsilon=65$, quoted by Pflaum and Brandt (1954). This method of analysis was sufficiently precise to allow proper matching of nuclear $T_{1}$ values for $\mathrm{Cu}\left(\mathrm{H}_{2} \mathrm{O}\right)_{6}^{2+}$ and the $\mathrm{VO}^{2+}$ solutions used in the flowing sample spectrometer. Nuclear $T_{1}$ values for $\mathrm{Cu}\left(\mathrm{H}_{2} \mathrm{O}\right)_{6}^{2+}$ which were used in the matching process were those measured by Morgan and Nolle (1959). DNP on $\mathrm{Cu}^{2+}$ was observed in both the flowing sample spectrometer and the moving sample tube spectrometer. In both cases the solutions used were $-0.03 \mathrm{M}$ in $\mathrm{Cu}^{2+}$. Measurements of enhancements for $\mathrm{Cu}^{2+}$ on 
the moving sample tube spectrometer were compared with $\mathrm{vo}^{2+}$, standards in the same manner as the $\mathrm{Cr}(\mathrm{CN})_{6}^{3-}$ measurements.

3. Results and Discussion

Enhancement spectra representative of those taken for $\mathrm{Cu}\left(\mathrm{H}_{2} \mathrm{O}\right)_{6}^{2+}$ with the moving sample tube spectrometer are shown in fig. 26. Spectra which were taken with the flowing sample spectrometer were similar, although the observed enhancements were about a factor of two smalier than those which were measured with the moving sample tube spectrometer. It can be seen in Fig. 26 that there is no sign of resolved hyperf ine structure, as would be expected from the appearance of the EPR spectra at these temperatures (Chang, 1971). There is no special significance to the relative amplitudes of the spectra shown in Fig. 26. Although both spectra were taken on solutions of the same $\mathrm{Cu}^{2+}$ concentration and at the same microwave power level, a number of parameters other than the saturation parameter which affect the enhancement are not independent of temperature. Both the cavity $Q$ and olo increase with temperature. Also the matching of the cavity to the transmission line is not necessarily constant with temperature. Finally, the spectra shown in Fig. 26 have not been adjusted for the effects of the finite microwave pulse length and the non-zero delay before measuring the proton magnatization. This adjustment is different for each spectrum since $T_{1 n}$ is a function of temperature.

Enhancements for the individual hyperf ine transitions were determined by a least squares fitting procedure similar to the one employed for $\mathrm{Cu}(\mathrm{en})_{2}\left(\mathrm{H}_{2} \mathrm{O}\right)_{2}^{2+}$. For $\mathrm{Cu}\left(\mathrm{H}_{2} \mathrm{O}\right)_{6}^{2+}$ it is not necessary to include 


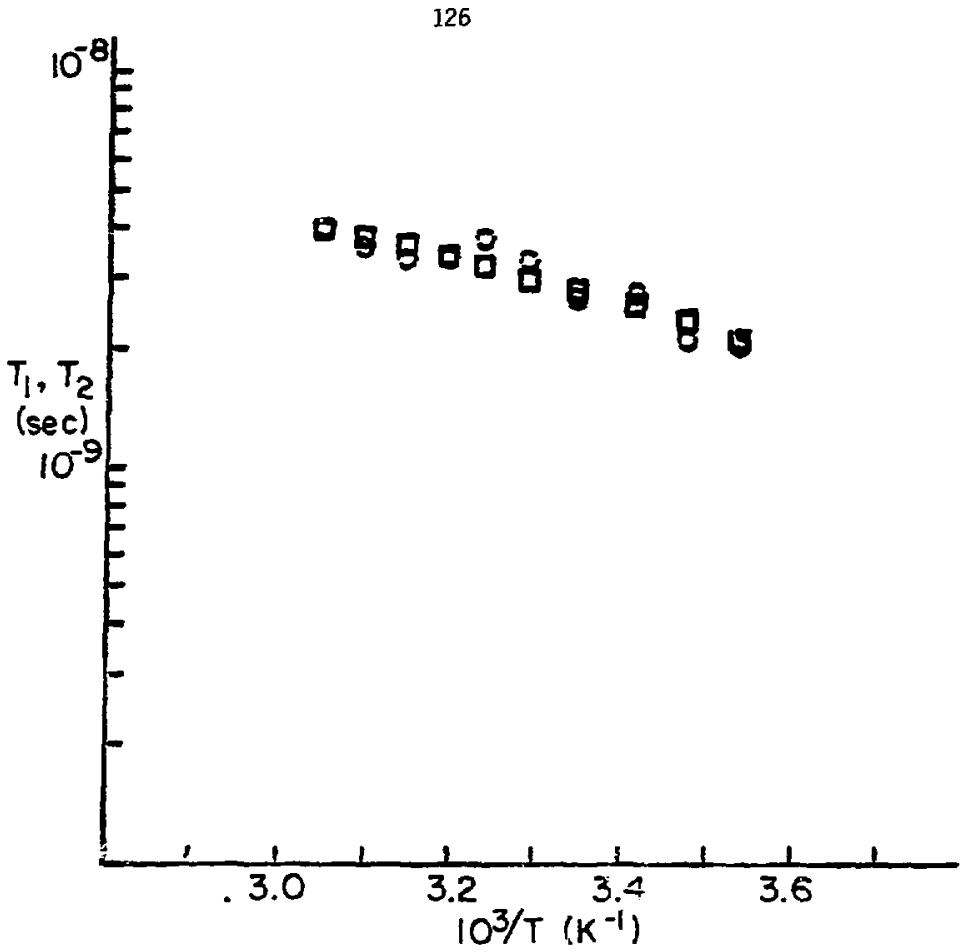

XBL $82.7-5968$

Fig. 25. $T_{1}$ and $T_{2}$ for $\operatorname{Cr}(C N)_{6}^{3-}$. Circles- $T_{1}$ from $D N P$ using $\sigma / \rho$ as described in the text. Squares-T $\mathrm{T}_{2}$ as in Fig. 24. 


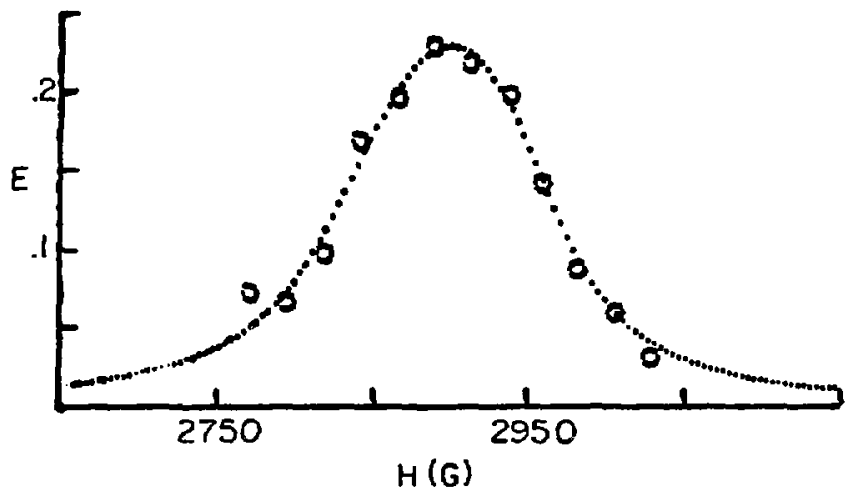

Fig. 26a. Enhancement spectrum of $\mathrm{Cu}\left(\mathrm{H}_{2} \mathrm{O}\right)_{6}^{2+}$ at $10^{\circ} \mathrm{C}$. The dotted line is a fit using four enhancements.

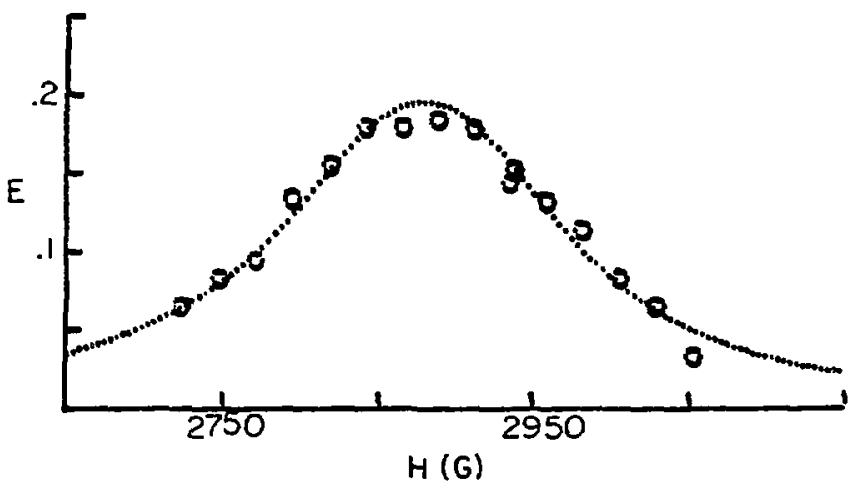

XBL 827- 5989

Fig. 26b. Enhancement spectrum of $\mathrm{Cu}\left(\mathrm{H}_{2} \mathrm{O}\right)_{6}^{2+}$ at $60^{\circ} \mathrm{C}$. The dotted line is a simulation using only a single enhancement. 
the effects of 1 igand superhyperf ine coupling. The proton superhyperfine coupling constant of $0.03 \mathrm{G}$ (Shulman and Luz, 1960) is small enough to be neglected. The results of the fitting process were disappointing, since there seemed to be no consistent pattern of $M_{I}$ dependence as a function of temperature. Indeed, at $60^{\circ} \mathrm{C}$ the best fit required one of the hyperf ine enhancements to be negative. It seems that the signal to noise was too poor, and that the hyperfine enhancements were too strongly correlated to give reliable fits. This was reinfarced by the fact that above $40^{\circ} \mathrm{C}$ the system became so illconditioned that fits could not be done in 36-bit floating-point arithmetic. Extension to 48 bits was necessary to get values for the hyperf ine enhancements which $f$ it the observed spectra, and these enhancements were suspect since one of them was negative. The consequence of this is that no comment on the low temperature $M_{I}$ dependent relaxation mechanism can be made from these data. In any case, it would require an exceptional signal to noise ratio to gain information on the tumbling/inversion mechanism. Estimates of the contribution of this mechanism to $1 / T_{1}$ made using Eqs. (4.1)-(4.5) indicate that it contributes on $7 y$ a few percent or less of $I / T_{1}$ at $X$-band, even at the lowest temperatures studied.

Because of the unreliability of the fitting process only an average $T_{1 e}$ could be determined for $\mathrm{Cu}\left(\mathrm{H}_{2} \mathrm{O}\right)_{6}^{2+}$. All of the enhancement spectra could be simulated reasonably well using a single enhancement obtained by simply averaging together the enhancements obtained from the fitting process for each hyperfine transition. These average 
enhancements were compared wi th enhancements measured for $\mathrm{vo}^{2+}$ to determine the average $T_{1 e^{*}}$ Average 1 inewidths and 1 inecenters for $\mathrm{Cu}\left(\mathrm{H}_{2} \mathrm{O}\right)_{6}^{2+}$, which were determined from measurements by Chang (1971), were used in the comparison process. The average $T_{1 e}$ values from enhancement data taken on the moving sample tube spectrometer are plotted in Fig. 27 and are compared with average $T_{2 e}$ values, Data were taken from $10^{\circ}$ to $60^{\circ} \mathrm{C}$ in $10^{\circ}$ intervals. The data from the flowing sample spectrometer, although iess extensive, are plotted for comparison in Fig. 27. The experimental error in these $T_{l e}$ values is estimated to be as large as 20 percent, mainly because the spread in the single enhancement which cauld reasonably simulate the enhancement spectrum is nearly that large. The o/p correction was made assuming ratational modulation of the dipolar interaction for $\mathrm{Cu}\left(\mathrm{H}_{2} \mathrm{O}\right)_{6}^{2^{+}}$. The hydrodynamic radius which was used in the correction, $2.8 \AA$, was determined from the measurements of the frequency cependence of proton NMR relaxation in $\mathrm{Cu}^{2+}$ solutions by Morgan and Nolle (1959).

The results in Fig. 27 are somewhat surprising, since it appears within experimental error that $T_{l e}$ is independent of temperature over this temperature range. Except for the spurious point at $3^{\circ} \mathrm{C}$, which was explained earlier in reference to $\mathrm{Cu}(\mathrm{en})_{2}\left(\mathrm{H}_{2} \mathrm{O}\right)_{6}^{2^{+}}$, the $\mathrm{T}_{1} \mathrm{e}$ values detemined from the flowing sample spectrometer show reasonable agreement with those frrm the moving sample tube spactrometer. What is particularly surprising is the fact that at $60^{\circ} \mathrm{C}$ we find $T_{1 e} \neq T_{2 e}$, while $T_{1 e}$ and $T_{2 e}$ approach each other at lower temperatures. This is the opposite of the behavior which was observed by McCain and Myers (1974) at S-band. 


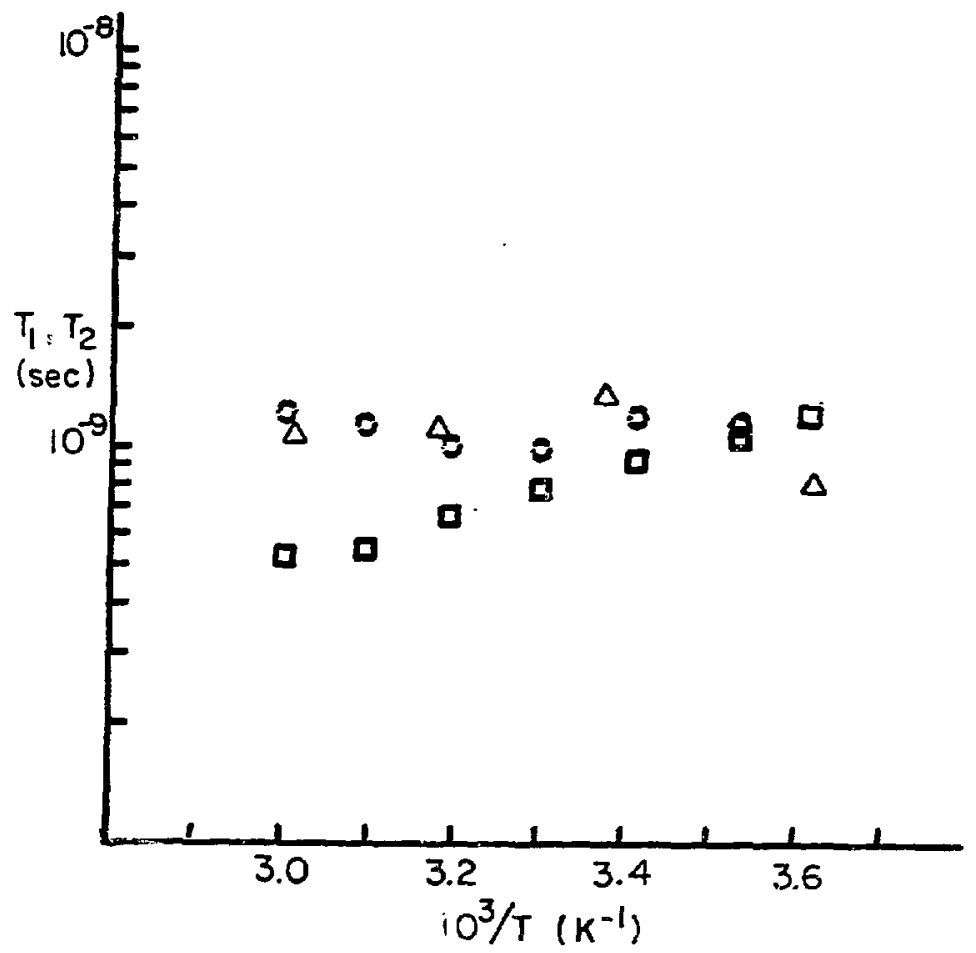

XBL $827-5990$

Fig. 27. $T_{1}$ and $T_{2}$ for $\mathrm{Cu}\left(\mathrm{H}_{2} \mathrm{O}\right)_{6}^{2+}$. Circles-T from moving sample tube DNP. Triangles-T from flowing sample ONP. The $a / \rho$ correction is described in the text. Squares $-T_{2}$ from 7 inewidth measurements by Chang (1971). 
The apparent 1 ack of temperature dependence of the measured $T_{1 e}$ values is difficult to reconcile with theory. In particular, a strong temperature dependent contribution to $1 / \tau_{1 e}$ is expected from the spin-rotation mechanism. One must conclude that spin-rotation is either masked by another relaxation mechanism, or that the measured $T_{1 e}$ values are incorrect due to some unforseen experimental error or an error in interpreting the data. The agreement between the $\mathrm{T}_{\text {le }}$ values measured using the two different spectrometers seems to argue against gross experimental error. The part of the data interpretation which is most subject to error is the o/p correction. It is clear from the $\mathrm{Cr}(\mathrm{CN})_{6}^{3-}$ data that a proper o/p correction is necessary to get a correct temperature dependence.

It has not been the policy in this work to treat o/p as an adjustable parameter. The o/p correction has always been made using experimentally detemined correlation times. However, in this case it is interesting to investigate the changes in tha temperature dependence of $T_{1}$ if the o/p correction is changed because the hydrodynamic radius for the $\mathrm{Cu}\left(\mathrm{H}_{2} \mathrm{O}\right)_{6}^{2+}$ complex obtained from $M R$ relaxation measurements is considerably smaller than the value expected from structural considerations. Perhaps this is because inversion affects the proton relaxation. Proton relaxation measurements have yielded values of 2.8A (Morgan and Nolle, 1959) and 2.3A (Frankel, 1968) for the hydrodynamic radius, while Meredith (1965) calculated a value of $3.65 \AA$ from $x-$ ray data on $\mathrm{CuSO}_{4} \cdot 5 \mathrm{H}_{2} \mathrm{O}$. 


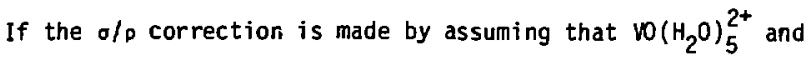
$\mathrm{Cu}\left(\mathrm{H}_{2} \mathrm{O}\right)_{6}^{2+}$ have the same hydrodynamic radius, the $\mathrm{T}_{1 \mathrm{e}}$ values plotted in Fig. 28 result. Chang (1971) has estimated a value of $3.67 \AA$ for the hydrodynamic radius of $\mathrm{WO}_{2}\left(\mathrm{H}_{2}\right)_{5}^{2+}$, which is near Meredith's structural value for $\mathrm{Cu}\left(\mathrm{H}_{2} \mathrm{O}\right)_{6}^{2+}$. This set of corrected $\mathrm{T}_{1 \mathrm{e}}$ values are interesting, because if $1 / T_{1 e}$ is plotted vs temperature divided by viscosity, the four lowest temperature points form a straight line which passes through the origin. This is the behavior expected if $T_{1}$ is dominated by spin rotation. Furthermore, from the slope of the line and Eqs. (2.8) and (2.5) a hydrodynamic radius of $3.5 \AA$ can be estimated. While this value is not completely consistent with the $\sigma / p$ correction, it is not in such poor agreement when one considers the approximate nature of the spin rotation theory. However, the two highest temperature points are not affected much by changes in the $\sigma / p$ correction, and do not lie on the spin-rotation line.

No matter which o/o correction is used we find $T_{1 e} \neq T_{2 e}$ at $60^{\circ} \mathrm{C}$. It is difficult to believe that this result is due to unforseen error, since it is easier to imagine reasons for the observed enhancement to be too small rather than too large. An additional unknown proton relaxation mechanism would cause smaller enhancements, as would relaxation by scalar coupling. It would be interesting to see if this behavior persists at higher temperatures. Higher temperature DNP measurements require technical improvements in the sealing of sample tubes to make the seals withstand the higher temperatures and pressures involved. If such improvements could be made the DNP results 


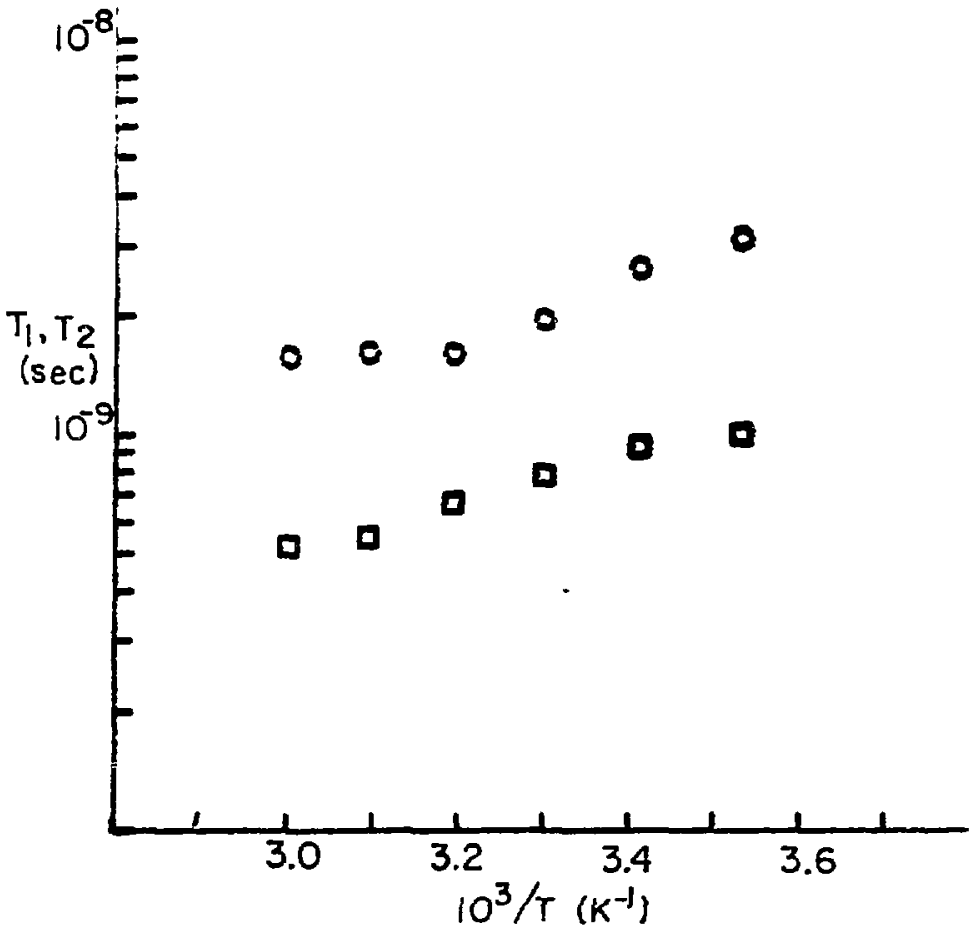

$\times B 2827-5991$

Fig. 28. $T_{1}$ and $\mathrm{T}_{2}$ for $\mathrm{Cu}\left(\mathrm{H}_{2} \mathrm{O}\right)_{6}^{2+}$. Circles-T from moving sample tube DNP? These are the same data as in Fig. 27 , but have a different $\sigma / \rho$ correction, which is described in the text. Squares-T2 as in Fig. 27. 
would be most useful, since the higher temperature points are much less sensitive to the o/p correction.

It is very difficult to draw definite conclusions from these $\mathrm{Cu}\left(\mathrm{H}_{2} \mathrm{O}\right)_{6}^{2+} \mathrm{T}_{1 \mathrm{e}}$ measurements. Surely the data rule out the importance of relaxation mechanisms such as those described by Al'tshuler and Valiev (1959) and Valiev and Zaripor (1964) which predict $T_{1 e} \gg T_{2 e}$. The lack of temperature dependence of the $T_{1 e}$ values which were calculated using MR data to determine the o/s correction seems unrealistic. The $T_{1 e}$ values calculated when the larger hydrodynamic radius estimated by Meredith (1965) is used in the o/o correction are more consistent with $T_{1 e}$ being determined by a spin-rotation interaction. However, it seems inappropriate to coment on the nature of the mechanism responsible for the difference between $T_{1 e}$ and $T_{2 e}$ which these data suggest, since there is no clear justification for the use of Meredith's hydrodynamic radius for $\mathrm{Cu}\left(\mathrm{H}_{2} \mathrm{O}\right)_{6}^{2+}$. Perhaps more useful DNP work could be done on $\mathrm{Cu}\left(\mathrm{H}_{2} \mathrm{O}\right)_{6}^{2+}$ at $\mathrm{S}$ band where the $\sigma / \mathrm{p}$ correction is not so important.

The electron spin relaxation in $\mathrm{Cu}\left(\mathrm{H}_{2} \mathrm{O}\right)_{6}^{2+}$ thus remains mysterious. We feel, however, that more accurate measurements of $T_{1 e}$ for this complex, whether made by DNP or some other method, can cantribute to the understanding of the structure and dynamics of this ion in solution. 
References

A. Abragam, The Principles of Nuclear Magnetism (Oxford University Press, London, 1961).

S. A. Al'tshuler and K. A. Valiev, Soviet Phys. JETP $\underline{35}, 661$ (1959).

N. S. Angerman and R. B. Jordan, J. Chem. Phys. 54, 837 (1971).

P. W. Atkins and D. Kiveison, J. Chem. Phys. 44, 169 (1966).

Y. I. Avvakumov, N. S. Garif'yanov, B. M. Kozyrev and P. G. Tishov, Soviet Phys. JETP 37, 1110 (1960).

D. M. S. Bagguley and J. H. E. Griffiths, Proc. Phys. Soc. (London) A65, 594 (1952).

G. Binch, Mo 1. Phys. 15, 469 (1968; .

J. Bjerrum and E. J. Nielson, Acta Chem. Scand. 2, 307 (1948).

F. Bloch, Phys. Rev. 70, 460 (1946).

N. Bloembergen and L. D. Morgan, J. Chem. Phys. 34, 842 (1961).

N. Bloembergen, E. M. Purcel 1, and R. V. Pound, Phys. Rev. 73, 679 (1948) .

L. Burlamacchi, G. Martini, M. F. Ottoviani and M. Romanelli, Adv. Ma). Int. 12, 145 (1978).

A. Carrington and G. R. Luckhurst, Mol. Phys. 8, 125 (1964).

J. J. Chang, Relaxation Time Measurements in Electron Paramagnetic Resonance (Ph. D. Thesis), UCRL-19691, February 1971.

R. S. Codrington and N. Bloembergen, J. Chem. Phys. 25, 1289 (1956).

G. F. Coster and H. Statz, Phys, Rev. 113, 445 (1959).

R. F. Curl, Mol. Phys. 9, 585 (1965).

L. S. Frankel, J. Phys. Chem. 12,736 (1968). 
J. H. Freed, J. Chem. Phys. 43, 2312 (1965).

J. H. Freed, Saturation and Double Resonance, in Electron Spin

Relaxation in Liquids, L. T. Muus and P. W. Atkins, eds. (Plenum -ress, New York, 1972).

S. Fu jiwara and H. Hayashi, J. Chem. Phys. 43, 23 (1965).

N. S. Garif'ianov and B. M. Kozyrev, Doklady ACad. Nauk. S.S.S.R. 98, 929 (1954).

B. B. Garrett and L. O. Morgan, J. Chem. Phys. 44, 890 (1966).

K. Güenther and H. Pfeifer, Zh. Strukt. Khim. 5, 193 (1964).

J. Haupt and W. Muller-Warmuth, Z. Naturforsch 17a, 1101 (1962).

K. H. hausser and D. Stehtik, Adv. Mag. Res. 3 , 79 (1968).

R. Hausser and G. Laukien, Z. Phys. 153, 394 (1959).

R. G. Hayes, Electron Spin Resonance Linewidths of Transition Metal Ions and Complexes in Solutions (Ph. D. Thesis), UCRL-9873, Jänuary 1961.

R. G. Hayes and R. J. Myers, J. Chem. Phys. 40,877 (1964).

P. S. Hubbard, Phys, Rev, 131, 1155 (1963).

P. K. Hurd, M. Sachs and W. D. Hershberger, Phys. Rev. 93, 373 (1954). M. M. Ianuzzi, C. P. Kubiak and P. H. Rieger, J. Phys. Chem. $80,5\{1$ (1976).

H. A. Jahn and E. Teller, Proc. Roy. Soc. Al61, 220 (1937).

D. Kivejson, J. Chem. Phys. 33, 1094 (1960).

D. Kivelson, J. Chem. Phys. 45, 1324 (1966).

B. M. Kozyrev, Dis. Faraday Soc. 19, 135 (1955). 
K. D. Kramer, W. Muller-Warmuth and J. Schindler, J. Chem. Phys. 43, 31 (1965).

R. Kubo and K. Tomita, J. Phys. Soc. Japan 9, 888 (1954).

V. Kul'beda, Soviet Physics JETP 19, 77 (1964).

J. S. Leigh, Jr., J. Mag. Res. 4, 308 (1971).

H. Levanon, S. Charbinsky and Z. Luz, J. Chem. Phys. 53, 3056 (1970).

W. B. Lewis, M. Alei, Jr. and L. 0. Morgan, J. Chem. Phys. 44, 2409 (1966a).

W. B. Lewis, M. Alei, Jr. and L. O. Morgan, J. Chem. Phys. 45, 4003 (1966b).

W. B. Lewis and L. O. Morgan, Paramagnetic Relaxation in Liquids, in Transition Metal Chemistry, Vol. 4, R. L. Carlin, ed. (Dekker, New York, 1968).

G. R. Luckhurst, ${ }^{6}$ S-State Ions, in Electron Spin Relaxation in Liquids, L. T. Muus and P. W. Atkins, eds. (Plenum Press, New York, 1972).

G. R. Luckhurst and G. F. Pedulli, Mo 1. Phys. 22, 931 (1971).

Z. Luz ano s. Meiboom, J. Chem. Phys. 40, 2686 (1964).

Z. Luz and R. G. Shulman, J. Chem. Phys. 43, 3750 (1965).

D. C. McCain, The Measurement of Electron Spin Relaxation Times in Aqueous So $1 r^{+} i \wedge n$ (Ph. D. Thes $i s$ ) UCRL-17064, August 1966.

D. C. McCain a. F. J. Myers, J. Phys. Chem. 71, 192 (1967).

D. C. MCCa in and R. J. Myers, Mol. Phys. 28, 1009 (1974).

H. M. McConne1 1, J. Chem. Phys. 25, 709 (1956).

H. M. McConne11, J. Chem. Phys. 28, 430 (1958). 
B. R. McGarvey, J. Phys. Chem. 61, 1232 (1957).

B. R. McGarvey, Electron Spin Resonance of Transition Metal Complexes, in Transition Metal Chemistry, Vol. 3, R. L. Carlin, ed. (Dekker, New York, 1966).

B. R. McGarvey, J. Phys. Chem. 71, 51 (1967).

C. W. Meredith, Temperature Dependence of Transverse Relaxation time of Oxygen-17 in Aqueous Solutions Containing Cupric and Chromous Ions (Ph. D. Thesis) UCRL-11704, January 1965.

L. O. Morgan, J. Murphy and P. F. Cox, J. Am. Chem. Soc. 81, 5043 (1959).

L. D, Morgan and A. W. Nolle, J. Chem. Phys. 31, 365 (1959).

W. Muller-Warmuth, Z. Naturforsch 18a, 1001 (1963).

M. Noack, G. F. Kokoszka and G. Gordon, J. Chem. Phys. 54, 1342 (1971).

M. C. M. O'Brien, Proc. Roy Soc. (London) A281, 323 (1964).

R. Orbach, Proc. Phys. Soc. (London) A77, 821 (1961).

A. W. Overhauser, Phys. Rev. 92, 411 (1953).

G. E. Pake and R. H. Sands, Phys. Rev. 98, 266 (1955).

H. Pfeifer, Z. Naturforsch 17a, 279 (1962).

R. T. Pflaum and W. C. Brandt, J. Am. Chem. Soc. 76, 6215 (1954).

C. P. Poole, Jr., Electron Spin Resonance (Wiley-Interscience, New York, 1967).

C. P. Poole, Jr., and H. A. Farrach, Relaxation in Magnetic Resonance (Academic Press, New York, 1971).

R. Poupko and Z. Luz, J. Lhem. Phys. 57, 3311 (1972).

A. G. Redfield, IBM J. Res. Develop. 1,19 (1957). 
J. Reuben and D. Fiat, Inorg. Chem, $\underline{8}, 1821$ (1969).

R. E. Richands and J. W. White, Proc. Roy. Soc. (London) A283, 459 (1964).

R. Santini, Jr., J. F. Hazel and W. M. McNabb, Anal. Chem. Acta. 6, 368 (1952).

W. W, Schmidt and K. G. Breitschwerdt, Chem. Phys, Lett. 그, 527 (1974).

D. Shoemaker, C. Garland and J. Steinfeld, Experiments in Physical Chemistry, 3rd ed., (McGraw-Hill, New York, 1974).

I. Solomon, Phys. Rev. 99, 559 (1955).

J. B. Spencer, Electron Spin Resonance Studies of Transition Metal Ion Complexes (Ph. D. Thesis), UCRL-16175, August 1965.

T. J. Swift and R. E. Connick, J. Chem. Phys. 37, 307 (1962).

M. Tinkham, R. Weinstein and A. F. Kip, Phys. Rev. 84, 848 (1951).

K. A. Valiev and M. M. Zaripov, Optics and Spectro. 20, 56 (1966).

D. Van Ormondt, R. de Beer, M. de Grant, Z. Naturf. A24, 1746 (1969).

J. H. Van Vleck, Phys. Rev. 57, 426 (1940).

R. C. Wilson, Electron Paramagnetic Resonance Studies of the Relaxation of Copper (II), Titanium (III) and Vanadyl Ions in Solution (Ph. D. Thesis), LBL-1462.

R. Wilson and D. Kivelson, J. Chem. Phys. $\underline{44}, 154$ (1966a).

R. Wilson and D. Kivelson, J. Chem. Phys. 44, 4445 (1966b).

K. Wuthrich and R. E. Connick, Inorg. Chem. 7, 1378 (1968). 
Acknowledgments

I would like to thank Professor Rollie J. Myers for the help and guidance which he provided throughout the course of this research. I would also like to thank the other members of the Myers group, Barrett Bobsein, James Roe, and Cindy Flanagan-Platenak, who gave the ir help whenever it was neederi. Dennis Reuter and Stephen Sears deserve special thanks for aiding in my introduction to the world of electronics and digital computers.

This work was supported by the Director, Office of Energy Research, Office of Basic Energy Sciences, Chemical Sciences Division of the US Department of Energy under Contract No. DE-ACO3-76SF00098. It was also partially supported by the Petroleum Resears'i Fund, administered by the American Chemical Society. Buch of these sources of support are gratefully acknowledged. 